\title{
Congress of Neurological Surgeons systematic review and evidence-based guidelines update on the role of targeted therapies and immunotherapies in the management of progressive glioblastoma
}

\author{
Evan Winograd ${ }^{1} \cdot$ Isabelle Germano $^{2} \cdot$ Patrick Wen $^{3} \cdot$ Jeffrey J. Olson ${ }^{4} \cdot$ D. Ryan Ormond ${ }^{1,5}$ (D)
}

Received: 8 September 2021 / Accepted: 11 October 2021 / Published online: 25 October 2021

(c) The Author(s), under exclusive licence to Springer Science+Business Media, LLC, part of Springer Nature 2021

\begin{abstract}
The following questions and recommendations are pertinent to the following:

Target population These recommendations apply to adults with progressive GBM who have undergone standard primary treatment with surgery and/or chemoradiation.

Question 1 In adults with progressive glioblastoma is the use of bevacizumab as monotherapy superior to standard salvage cytotoxic chemotherapy as measured by progression free survival and overall survival?

Recommendation Level III: Treatment with bevacizumab is suggested in the treatment of progressive GBM, as it provides improved disease control compared to historical controls as measured by best imaging response and progression free survival at 6 months, while not providing evidence for improvement in overall survival.

Question 2 In adults with progressive glioblastoma is the use of bevacizumab as combination therapy with cytotoxic agents superior to standard salvage cytotoxic chemotherapy as measured by progression free survival and overall survival?

Recommendation Level III: There is insufficient evidence to show benefit or harm of bevacizumab in combination with cytotoxic therapies in progressive glioblastoma due to a lack of evidence supporting a clearly defined benefit without significant toxicity.

Question 3 In adults with progressive glioblastoma is the use of bevacizumab as a combination therapy with targeted agents superior to standard salvage cytotoxic chemotherapy as measured by progression free survival and overall survival?

Recommendation There is insufficient evidence to support a recommendation regarding this question.

Question 4 In adults with progressive glioblastoma is the use of targeted agents as monotherapy superior to standard salvage cytotoxic chemotherapy as measured by progression free survival and overall survival?

Recommendation There is insufficient evidence to support a recommendation regarding this question.

Question 5 In adults with progressive glioblastoma is the use of targeted agents in combination with cytotoxic therapies superior to standard salvage cytotoxic chemotherapy as measured by progression free survival and overall survival?

Recommendation There is insufficient evidence to support a recommendation regarding this question.

Question 6 In adults with progressive glioblastoma is the use of immunotherapy monotherapy superior to standard salvage cytotoxic chemotherapy as measured by progression free survival and overall survival?

Recommendation There is insufficient evidence to support a recommendation regarding this question.

Question 7 In adults with progressive glioblastoma is the use of immunotherapy in combination with targeted agents superior to standard salvage cytotoxic chemotherapy as measured by progression free survival and overall survival?
\end{abstract}

Sponsors: Congress of Neurological Surgeons (CNS) and the Section on Tumors.

Endorsement: Reviewed for evidence-based integrity and endorsed by the American Association of Neurological Surgeons and Congress of Neurological Surgeons.

Extended author information available on the last page of the article 
Recommendation There is insufficient evidence to support a recommendation regarding this question.

Question 8 In adults with progressive glioblastoma is the use of immunotherapy in combination with bevacizumab superior to standard salvage cytotoxic chemotherapy as measured by progression free survival and overall survival?

Recommendation There is insufficient evidence to support a recommendation regarding this question.

Keywords Progressive glioblastoma $\cdot$ Guidelines update $\cdot$ Targeted therapy $\cdot$ Immunotherapy

\section{Abbreviations}

\section{BEV Bevacizumab \\ GBM Glioblastoma}

PFS Progression free survival

PFS6 6 month progression free survival

mOS Median overall survival

OS Overall survival

PRISMA Preferred reporting items for systematic

reviews and meta-analyses

\section{Introduction}

In 2014, guidelines for the management of progressive glioblastoma and the role of targeted therapies were published by the AANS and CNS [1]. We now present the updated set of data and studies regarding targeted therapies as well as immunotherapy with and without targeted therapies. Despite maximal therapy, the median overall survival for a patient with newly diagnosed glioblastoma remains 14.6 months [2]. Recurrence typically occurs by one year and the median overall survival at recurrence ranges from 24 to 44 weeks [3-5]. Despite significant effort, the prognosis after recurrence in glioblastoma remains dismal. While cytotoxic therapies have long been used to treat malignancy, more recent attempts utilizing therapies targeting tumor progression and growth pathways, anti-angiogenic agents designed to target neovascular proliferation pathways, and immunotherapies aimed at utilizing a patient's immune system to attack tumor cells have been studied. We present a systematic review and evidence-based practice guideline to help practicing physicians to determine the role of these treatments in progressive glioblastoma.

\section{Rationale}

Tumor stabilization and radiographic response are the current goals in designing treatment of progressive glioblastoma. There are several strategies for treatment with targeted therapies, especially with the large amount of information being uncovered regarding the molecular characteristics of glioblastoma and the differing aberrations that occur in subgroups of the tumor. Exploration of immunotherapy has been a growing subject of study as well, with a goal of utilizing the patient's own immune response to attack the tumor.

\section{Objectives}

The objectives of this guideline are to assess the therapeutic value of targeted therapies and immunotherapies in patients with progressive glioblastoma based on radiographic response and in terms of survival outcomes.

\section{Methods}

\section{Writing group and question establishment}

The evidence-based clinical practice guideline taskforce members and the Joint Tumor Section of the American Association of Neurological Surgeons (AANS) and the Congress of Neurological Surgeons (CNS) have prioritized updating the guidelines for the management of progressive glioblastoma with targeted therapies and immunotherapy. A series of authors for the development of guidelines related to the role of targeted therapy and immunotherapy in progressive glioblastoma were identified and screened for conflicts of interest. This group in turn agreed on a set of questions addressing the topic at hand and conducted a systematic review of the literature relevant to management of progressive glioblastoma with the aforementioned therapeutic strategies, both alone and in combination. Additional details of the systematic review are provided below and within the introduction and methodology chapter of the guideline.

\section{Literature search}

The task force collaborated with a medical librarian and searched for articles published on targeted therapies for progressive glioblastoma between July 1, 2012 and March 31, 2019, and on immunotherapy for progressive glioblastoma between January 1, 1990 to March 31, 2019 (to complete the same search dates as had previously been performed in the original guideline document) in three electronic databases: Pubmed, EMBASE and Cochrane. Strategies for searching electronic databases were constructed by the evidence-based clinical practice guideline taskforce members and the medical librarian using standard strategies to identify relevant 
studies [6-13]. As can be seen below, progression, recurrence and relapse were all used in the search strategy. As progression clearly implies glioblastoma persists and is not completely controlled with any intervention, this term is chosen as the primary term describing the clinical circumstance addressed throughout this guideline.

\section{Search strategy}

- Targeted: July 1, 2012 through March 31, 2019

o (progress* OR recurren* OR relaps*) AND (glioma OR glioblastoma) AND (targeted therap* OR immunotherapy OR molecular agent* OR surgical technique* OR surgery OR radiotherap* OR alternative particles OR radiosensitizer* OR convection enhanced) AND (quality of life OR survival OR mortality)

- Immunotherapy: January 1, 1990 to March 31, 2019

o (progress* OR recurren* OR relaps*) AND (glioma OR glioblastoma) AND (vaccin* OR peptide vaccin* OR heat shock protein vaccin* OR immune checkpoint inhibitor* OR dendritic cell vaccin* OR adoptive T Cell*) AND (quality of life OR surival OR mortality)

The authors supplemented the searches of electronic databases with manual screening of the bibliographies of all retrieved publications. The authors also searched the bibliographies of recent systematic reviews and other review articles for potentially relevant citations. All articles identified were subject to the study selection criteria listed below. As noted above, the guideline committee also examined lists of included and excluded studies for errors and omissions. The authors went to great lengths to obtain a complete set of relevant articles. Having a complete set ensured that this guideline was not based on a biased subset of articles.

\section{Study selection and eligibility criteria}

A total of 4417 articles were identified including 3513 for targeted therapies and 904 for immunotherapy (Figs. 1 and 2). Based on a Pubmed search, a total of 2727 articles in targeted therapy and 627 articles in immunotherapy related to progressive glioblastoma were reviewed by the team with specific inclusion and exclusion criteria outlined below. A search of Embase yielded 748 additional articles relating to targeted therapy and 253 in immunotherapy. Cochrane yielded an additional 38 articles for targeted therapy and 24 for immunotherapy. The same methodology of review was conducted for the Embase and Cochrane data. Independent reviewers evaluated and abstracted full-text data for each article and the two sets of data were compared for agreement by a third party. Inconsistencies were re-reviewed and disagreements were resolved by consensus. Citations were included that prospectively or retrospectively reviewed the

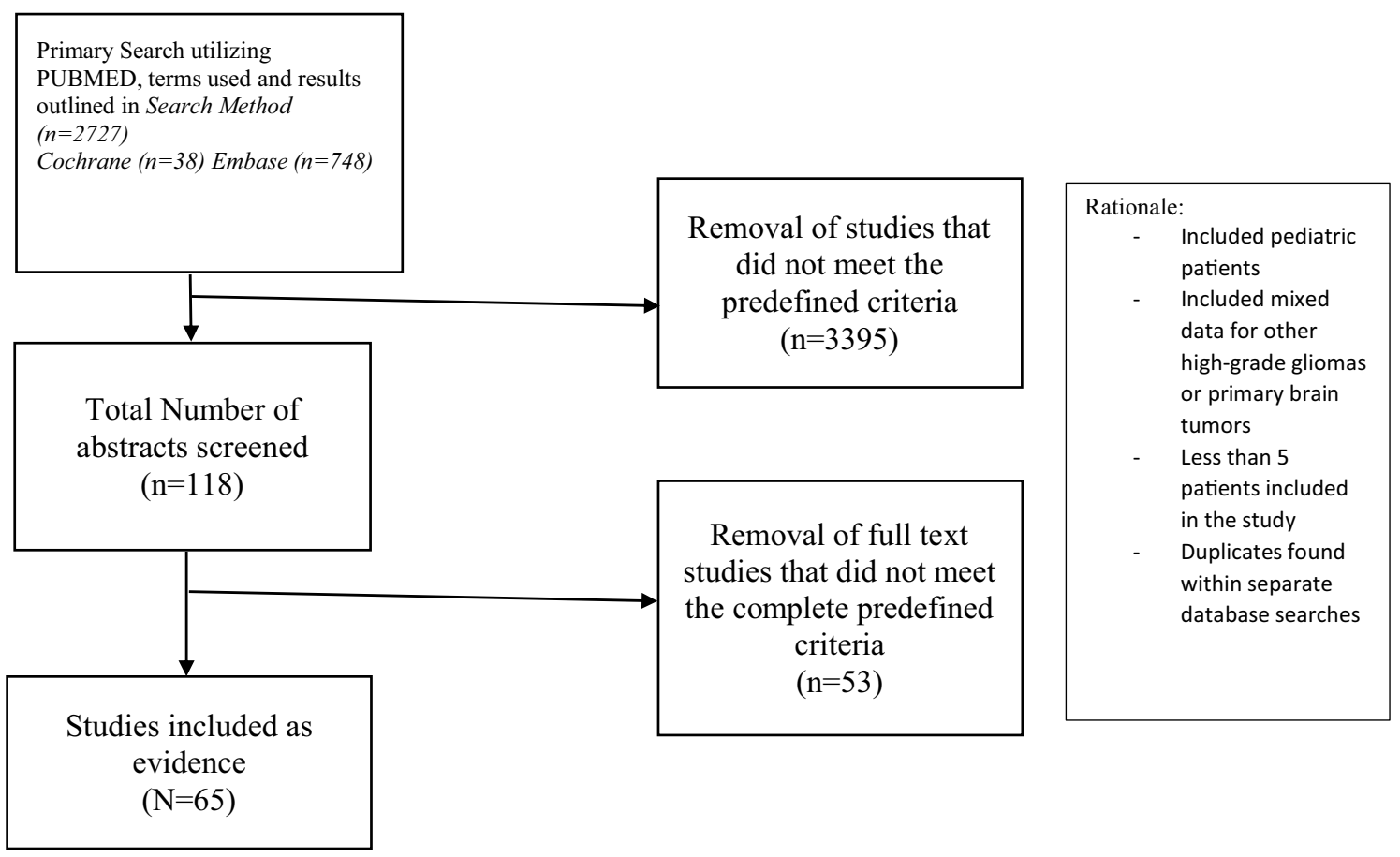

Fig. 1 PRISMA diagram — depicting the process of searching for, including, and excluding (as well as rationale) studies on targeted therapies for treating progressive glioblastoma 


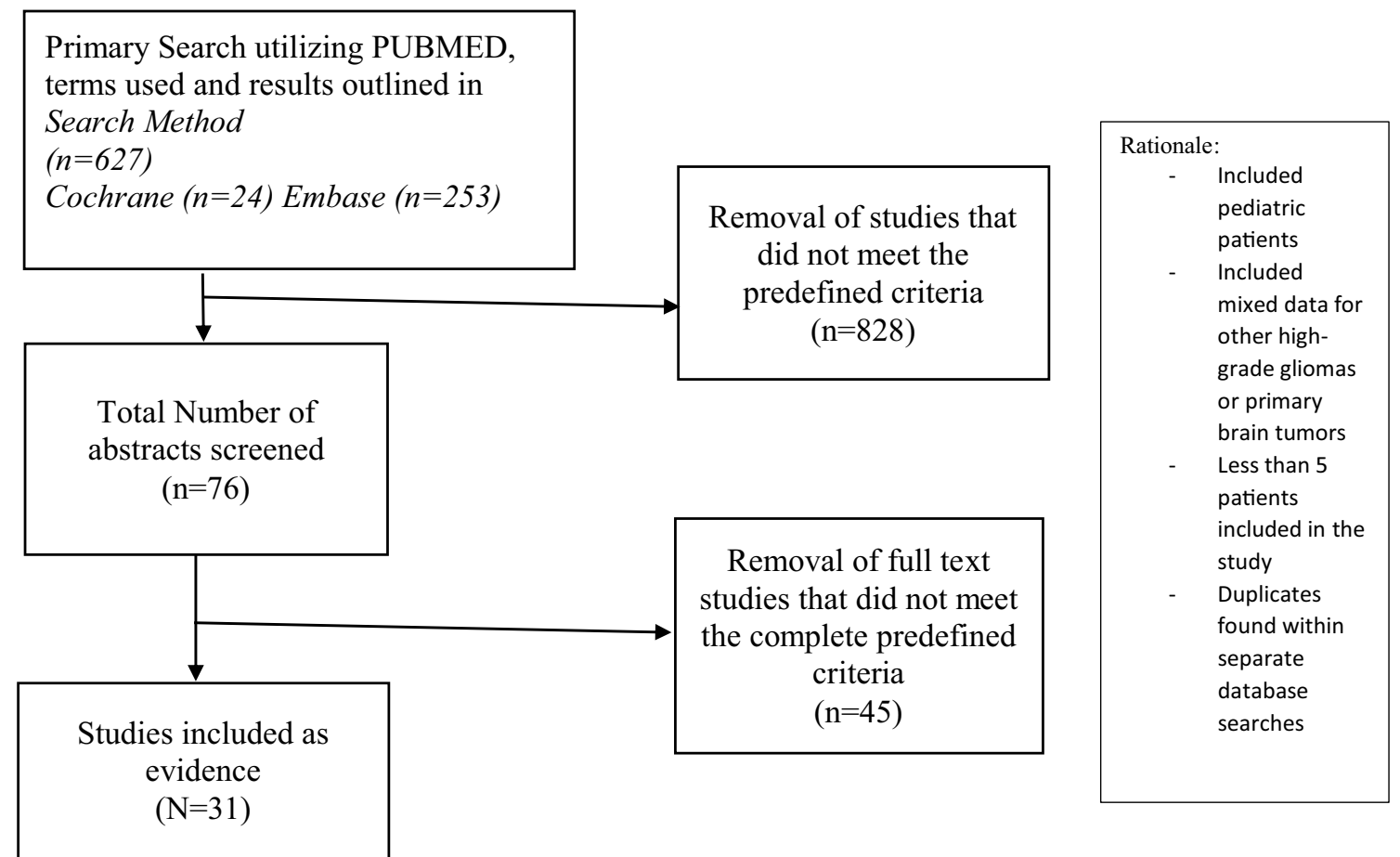

Fig. 2 PRISMA diagram-depicting the process of searching for, including, and excluding (as well as rationale) studies on immunotherapy for treating progressive glioblastoma

treatment effect and outcomes of targeted therapies and immunotherapies, as monotherapy or combination therapies, on progressive glioblastoma patients. To be included in the guideline, a publication had to meet the following inclusion criteria:

- Investigated patients had progressive/recurrent glioblastoma

- Patients $\geq 18$ years of age

- Humans, only

- Published between:

o Targeted: July 1, 2012 through March 31, 2019

o Immunotherapy: January 1, 1990 to March 31, 2019

- Quantitatively presented results

- Was not an in vitro study

- Was published in English

- Studies may include mixed pathology; however, the data pertaining to progressive glioblastoma was extractable from the paper

- $\mathrm{Had} \geq 5$ patients or patient samples

The authors did not include systematic reviews, guidelines, or meta-analyses conducted by others. These documents are developed using different inclusion criteria than those specified in this guideline. Therefore, they may include studies that do not meet the inclusion criteria specified above. These documents were recalled if their abstract suggested that they might address one of the recommendations, and their bibliographies were searched for additional studies.

\section{Data collection process}

The abstracts that met the selection criteria mentioned above were retrieved in full-text form. Each article's adherence to the selection criteria was confirmed. To determine how the data could be classified, the information in the full-text articles were then evaluated to determine whether they were providing results of therapy or were more centered on diagnostic or prognostic information. Agreement on these assessments and on the salient points regarding the type of study design and objectives, and the conclusions and data classification was then reached by exchanging drafts and comments by e-mail. The information was then used for construction of the evidence tables. A summary of the search process and results are provided in the PRISMA diagrams (Figs. 1 and 2).

\section{Assessment for risk of bias}

Our search generated 4417 abstracts. These were screened, and the articles that addressed our pre-defined questions 
underwent full independent review by the authors. Reviewers were critical in their assessment to account for selection bias, survivorship bias, etc., by evaluating studies specifically in regard to trial design including factors such as: adequacy of control group, randomization of treatment, blinding, prospective character, size of study population, etc. Other factors that could account for survivorship bias or selection bias, as well as appropriate or inappropriate use of statistics of the reported data was also critically assessed.

\section{Classification of evidence and guideline recommendation and formulation}

The concept of linking evidence to recommendations has been further formalized by the American Medical Association (AMA) and many specialty societies, including the American Association of Neurological Surgeons (AANS), the Congress of Neurological Surgeons (CNS), and the American Academy of Neurology (AAN). This formalization involves the designation of specific relationships between the strength of evidence and the strength of recommendations to avoid ambiguity. In the paradigm for therapeutic maneuvers, evidence is classified into that which is derived from the strongest clinical studies (e.g., welldesigned, randomized controlled trials), or class I evidence. Class I evidence is used to support recommendations of the strongest type, defined as level 1 recommendations, indicating a high degree of clinical certainty. Nonrandomized cohort studies, randomized controlled trials with design flaws, and case-control studies (comparative studies with less strength) are designated as class II evidence. These are used to support recommendations defined as level 2 reflecting a moderate degree of clinical certainty. Other sources of information, including observational studies such as case series and expert opinion, as well as randomized controlled trials with flaws so serious that the conclusions of the study are truly in doubt are considered class III evidence and support level 3 recommendations, reflecting unclear clinical certainty. A summary of these categories of evidence can be viewed at https://www.cns.org/guidelines/guideline-devel opment-methodology.

\section{Results}

\section{Role of targeted therapies in progressive glioblastoma}

All questions and recommendations in this section apply to the following target population: Adults with progressive GBM who have undergone standard primary treatment with surgery and/or chemoradiation. For each of the provided evidence tables, data extraction included study design, total number of patients, study parameters including treatment regimen, results including metrics of survival and progression free survival, complications including toxicity data, and author conclusions.

Question 1 In adults with progressive glioblastoma is the use of bevacizumab as monotherapy superior to standard salvage cytotoxic chemotherapy as measured by progression free survival and overall survival?

\section{Summary of prior recommendations}

In the previously published guidelines on the role of targeted therapies in the management of progressive glioblastoma in adults, it was recommended that bevacizumab be utilized to provide improved disease control in comparison to historical controls as measured by best imaging response and progression free survival at 6 months [1]. This was a Level III recommendation based on the available data. The previous data included both prospective trials and retrospective reviews, all of which were Class III evidence [1].

\section{Recommendation: Level III}

Treatment with bevacizumab is suggested in the treatment of progressive GBM, as it provides improved disease control compared to historical controls as measured by best imaging response and progression free survival at 6 months, while not providing evidence for improvement in overall survival.

\section{Study selection and characteristics for the updated search}

The initial search strategy included 3513 candidate articles. A total of 65 articles remained for full text review. From these, 7 articles were included in the final review for Question 1 and are included in Table 1 [14-20].

Only two of the studies were prospective trials. One was performed in Japan, by Nagane et al. [17] They evaluated 29 progressive glioblastoma patients given a regimen of standard dose BEV and found mOS was 10.5 months. They had a significant number of radiographic responses with 21 patients experiencing reduction in tumor size during treatment and a PFS 6 of $33.9 \%$. They concluded that, similar to other studies performed outside Japan, their native patient population with progressive glioblastoma experienced a significant survival benefit [21-23]. Cai et al. reviewed 20 patients in a double arm comparative study comparing BEV to a placebo treatment [14]. Of the patients with glioblastoma $(n=20)$, mOS was 8.9 months with BEV and 5.6 months with placebo, and a 6 month survival of $83 \%$ versus $47 \%$ respectively. The authors concluded that bevacizumab conferred a survival benefit. 


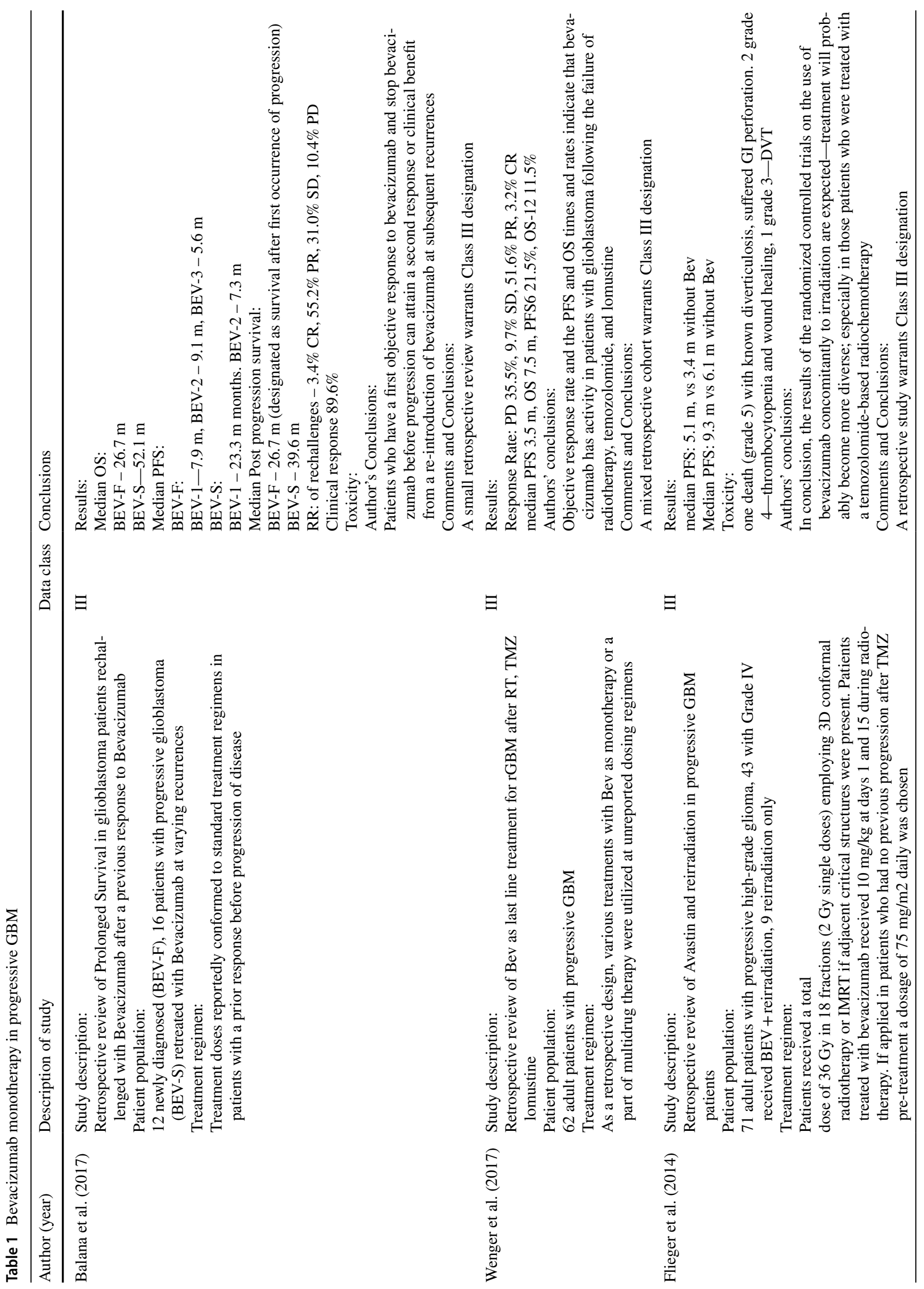




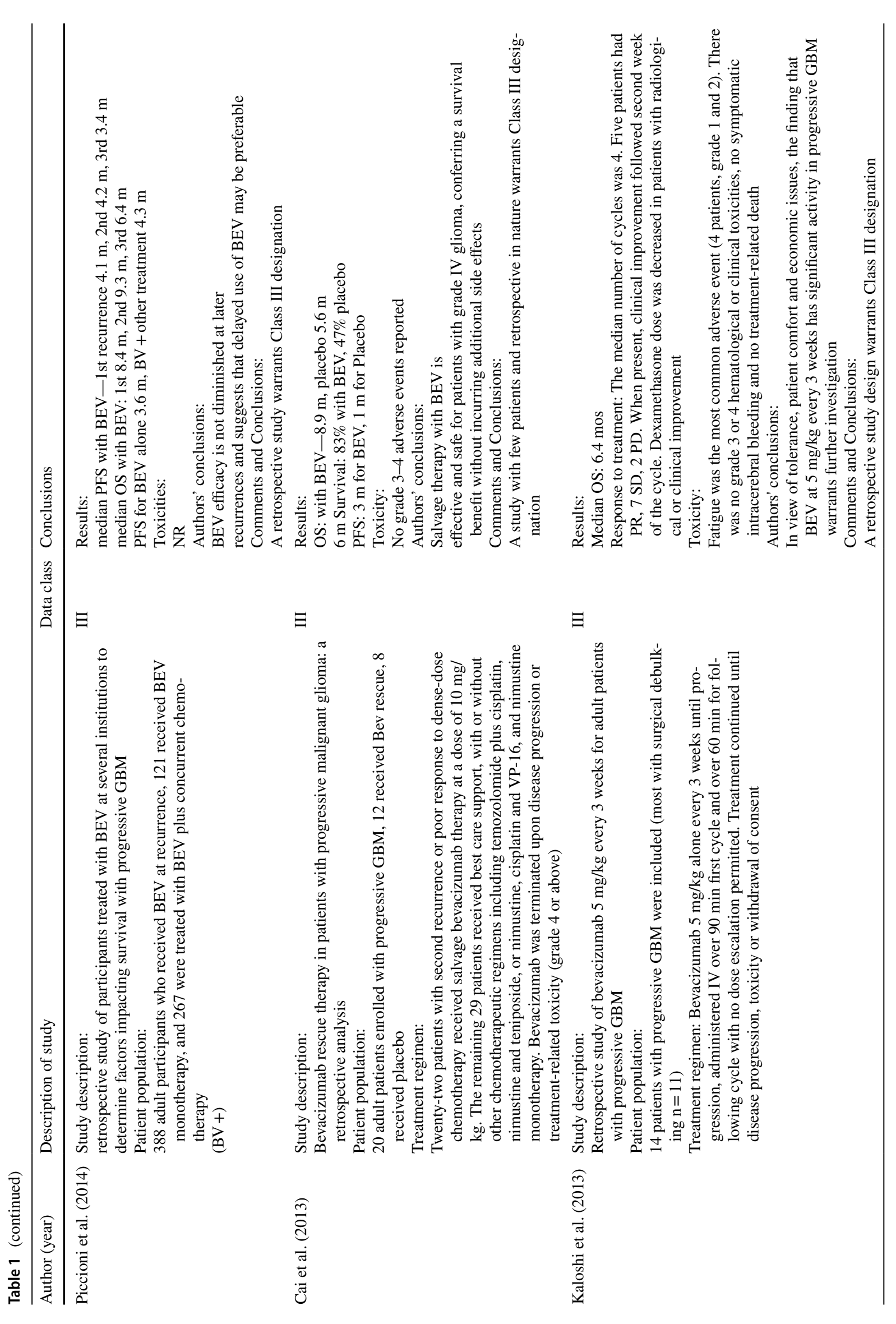




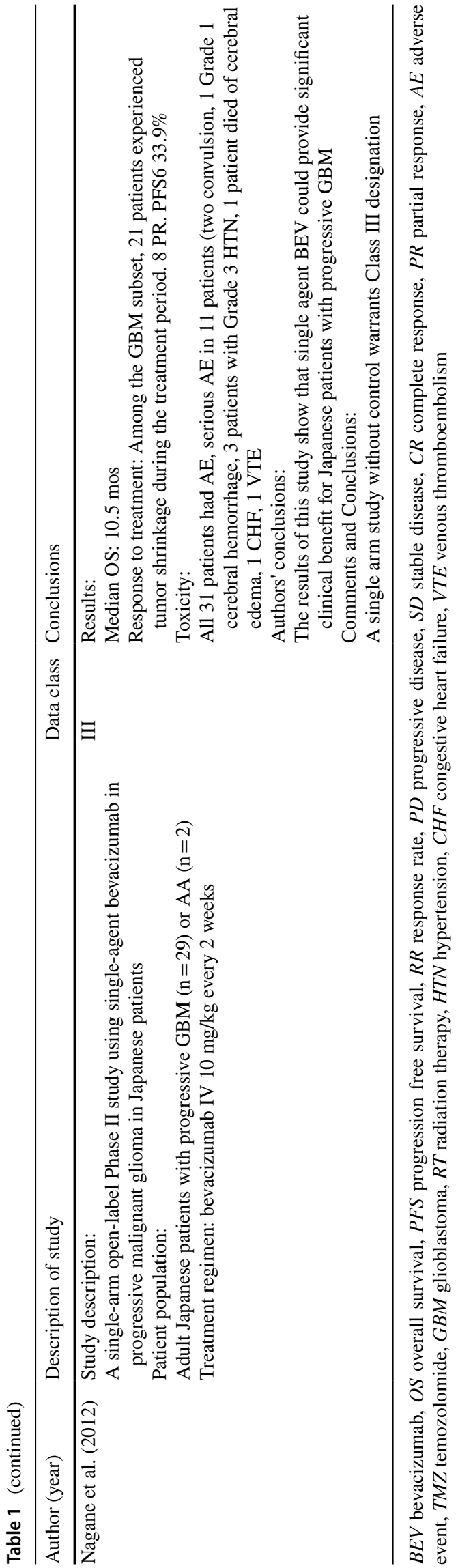

Six of the studies were retrospective reviews. [14-16, 18-20] Kaloshi et al. evaluated the survival effect on 11 patients receiving low-dose BEV, finding mOS of 6.4 months and only five patients with a partial response, at best. The coupling of low toxicity and some efficacy lead them to conclude BEV was effective in these patients [16]. Wenger et al. reviewed 62 patients with multiple treatment regimens, however all had undergone and failed initial standard therapy with RT, TMZ, and lomustine [19]. Ultimately, their RR showed $51.6 \%$ PR and $3.2 \%$ with $\mathrm{CR}$, a median PFS of 3.5 months and mOS 7.5 months. They felt this data showed BEV remained effective after failed standard treatment and lomustine. Meanwhile, Piccioni et al. reviewed data from multiple institutions including 388 patients receiving BEV at recurrence, however in varying methodologies including combination therapy [18]. Progression free survival for BEV alone was 3.6 months and 4.6 months in combination therapy. Their data showed that BEV retained its efficacy throughout multiple recurrences, with data including the varying recurrences at which BEV was started, however the data was mixed with multiple therapy modalities. Balana et al. also studied patients rechallenged with BEV after prior treatment response to the drug, and noted a survival benefit, possibly in the subset of patients that displayed sensitivity and response to initial treatment with BEV, if BEV was halted for reasons other than progression [20]. Flieger et al. reviewed their data on patients receiving repeat RT therapy along with BEV [15]. PFS was 5.1 months with and 3.4 months without bevacizumab. Median OS was 9.3 months in combination therapy, 6.1 months with RT alone and the authors concluded that BEV concomitantly given with reirradiation was feasible and effective based on comparison to historical data.

\section{Synthesis}

Historically, PFS-6 ranged from 29 to $50.3 \%$ based on three studies evaluating BEV monotherapy compared with combination therapy with irinotecan, with Friedman et al. showing in a prospective, randomized Phase II trial a mOS of 9.2 months with BEV alone [22-24]. Two prospective trials with BEV above showed a PFS- 6 of $33.9 \%$ and $83 \%$, compatible with the historical data [17]. In one, the data was a single arm study without a control group, and the other a small case series where the controls fared better than controls in other studies. For this reason, these prospective studies remained Class III evidence. The remainder of the data was retrospective, all of which were downgraded to Class III evidence, as well [14-19]. While these studies met the inclusion criteria, they did not provide enough high level evidence to change or upgrade the recommendation from the previous guideline. 
Question 2 In adults with progressive glioblastoma is the use of bevacizumab as combination therapy with cytotoxic agents superior to standard salvage cytotoxic chemotherapy as measured by progression free survival and overall survival?

Prior Recommendation: In the previously published guidelines on the role of targeted therapies in the management of progressive glioblastoma in adults, bevacizumab combined with cytotoxic agents was reviewed and no specific recommendations were made based on a lack of strong evidence supporting a clearly defined benefit without significant toxicity [1].

\section{Recommendation Level: III}

There is insufficient evidence to show benefit or harm of bevacizumab in combination with cytotoxic therapies in progressive glioblastoma due to a lack of evidence supporting a clearly defined benefit without significant toxicity.

\section{Study selection and characteristics of the updated search}

The initial search strategy included 3513 candidate articles. A total of 65 articles remained for full text review. From these, 21 articles were included in the final review for Question 2 and are included in Table 2 [25-44].

\section{Bevacizumab with cytotoxic therapy}

There were six studies regarding BEV combined with cytotoxic therapy included in our search that met criteria for inclusion. In a small series, Arakawa et al. evaluated combination therapy with ifosfamide, carboplatin, and etoposide at second recurrence, finding an mOS of 6 months [25]. In a similar study, Brenner et al. added evofosfamide to BEV in BEV resistant patients compared to placebo, finding a mOS of 4.4 months with SD in $60.9 \%$ and a response in $17.4 \%$ [29]. They found preliminary evidence of synergistic activity. Two further studies combined TMZ with BEV. Badruddoja et al. combined BEV with TMZ and found a mOS of 11.0 months and PFS6 of 52\% [26]. Sepulveda et al. similarly studied BEV with TMZ and found a PFS6 of $21.9 \%$, finding the regimen was safe and possibly effective [39]. Another study by Peters et al. combined Vorinostat, BEV, and TMZ at recurrence, but found their results limited by their cohort's heavy pretreatment prior to enrollment [37]. Mrugala et al. retrospectively studied BEV with carboplatin at varying recurrences, finding a mOS of 9.3 months, determining some activity and tolerability of the combined therapy [44]. Ultimately, the data here regarding cytotoxic therapy with BEV at recurrence warranted Class III data designation as the studies were small and/or lacked randomization.

However, there was one study that yielded class II evidence. Field et al. randomized 122 patients with BEV and carboplatin versus BEV monotherapy [30]. PFS6 was $15 \%$ in combination therapy, $18 \%$ in monotherapy, thus they found no additional benefit with combination therapy.

\section{Nitrosoureas with bevacizumab}

The only Class I designation was afforded to Wick et al. for evaluating combination therapy with BEV and lomustine as a prospective, randomized phase III trial in 437 adult progressive glioblastoma patients [43]. Monotherapy OS was 8.6 months and PFS 1.5 months. Combination therapy OS was 9.1 months and PFS 4.2 months. The trial concluded there was not a survival advantage using combination therapy beyond that obtained with monotherapy.

Brandes et al. performed a phase II randomized trial of 91 patients receiving fotemustine or BEV for progressive glioblastoma [27]. The authors concluded there may be a role for single agent $\mathrm{BEV}$ in progressive glioblastoma as median OS was 7.3 months with BEV and 8.7 months with fotemustine. RANO response rates were $29 \%$ with BEV and $9 \%$ with fotemustine. They had also completed a phase II randomized and double blinded study on 123 patients given BEV and Lomustine or Lomustine with placebo in first recurrence of glioblastoma [28]. At second recurrence or progression of disease, patients continued BEV or placebo. Results showed CCNU + BEV median overall survival (mOS) to be 6.4 months, PFS2 2.3 months, PFS3 2.0 months (PFS2 and PFS3 refer to the progression free survival at the second and third recurrence, respectively). There was no survival benefit and no detriment determined by the authors in continuing BEV through multiple lines of treatment. These were both designated Class II data as they were prospective, randomized trials with small cohorts.

In another randomized, controlled, Phase II study, Taal et al. evaluated BEV with lomustine vs BEV monotherapy or lomustine monotherapy in 153 patients [40]. Combination therapy showed an improved OS and treatment response with 9 months OS in 63\%, surpassing the monotherapy groups. Authors concluded the combination treatment met their predetermined criteria for further evaluation. Another study by Weathers et al. was a randomized phase II trial of BEV standard dose vs low dose BEV with lomustine, which found nonsuperiority of combination therapy [42]. These studies warranted Class II designation.

The remainder of the data was Class III as the cohorts were small, the data was retrospective, or the study lacked a control group. Heiland et al. retrospectively reviewed BEV with lomustine against BEV monotherapy in 35 patients [33]. Median OS was 6.6 months with improved median 


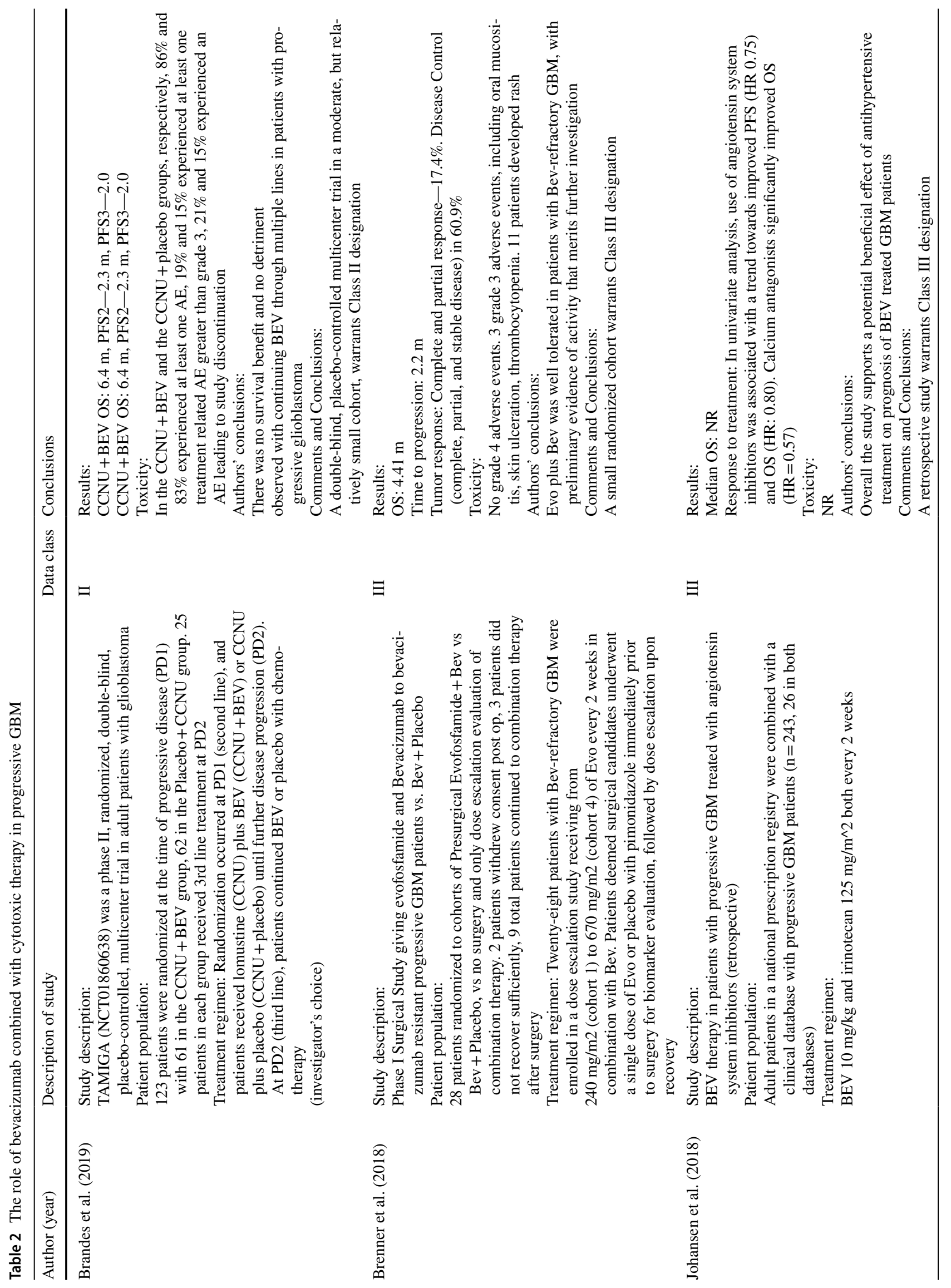




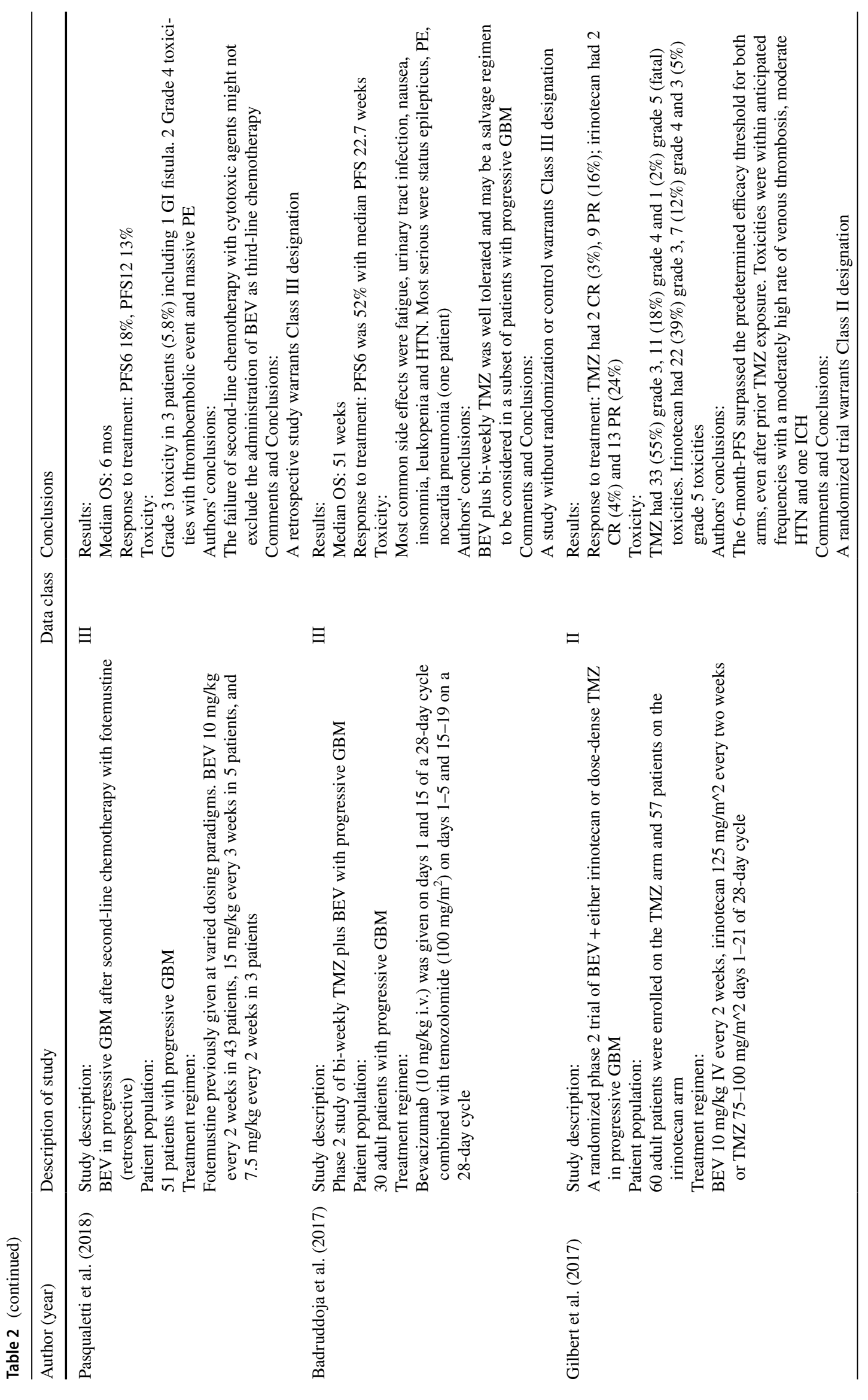




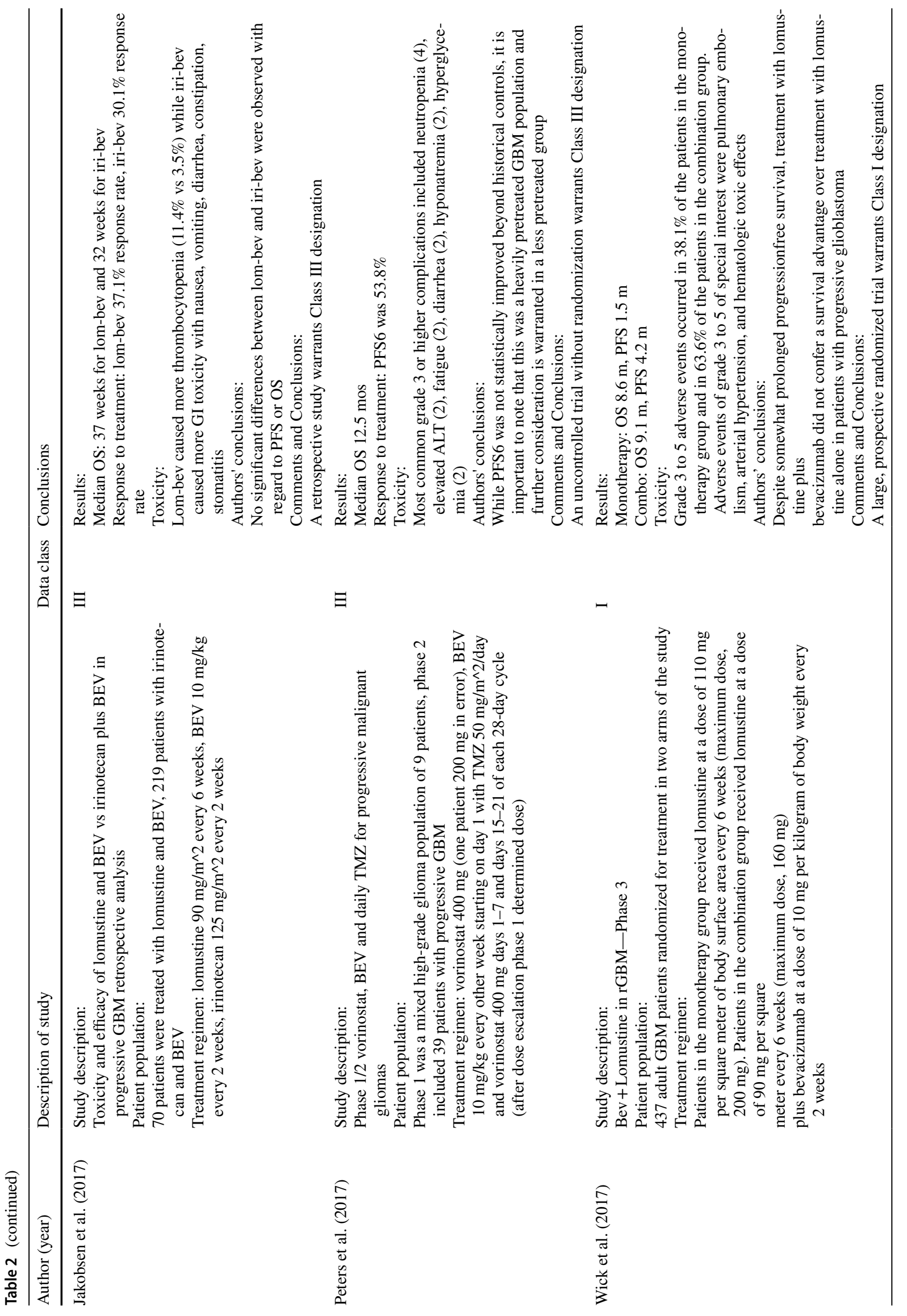




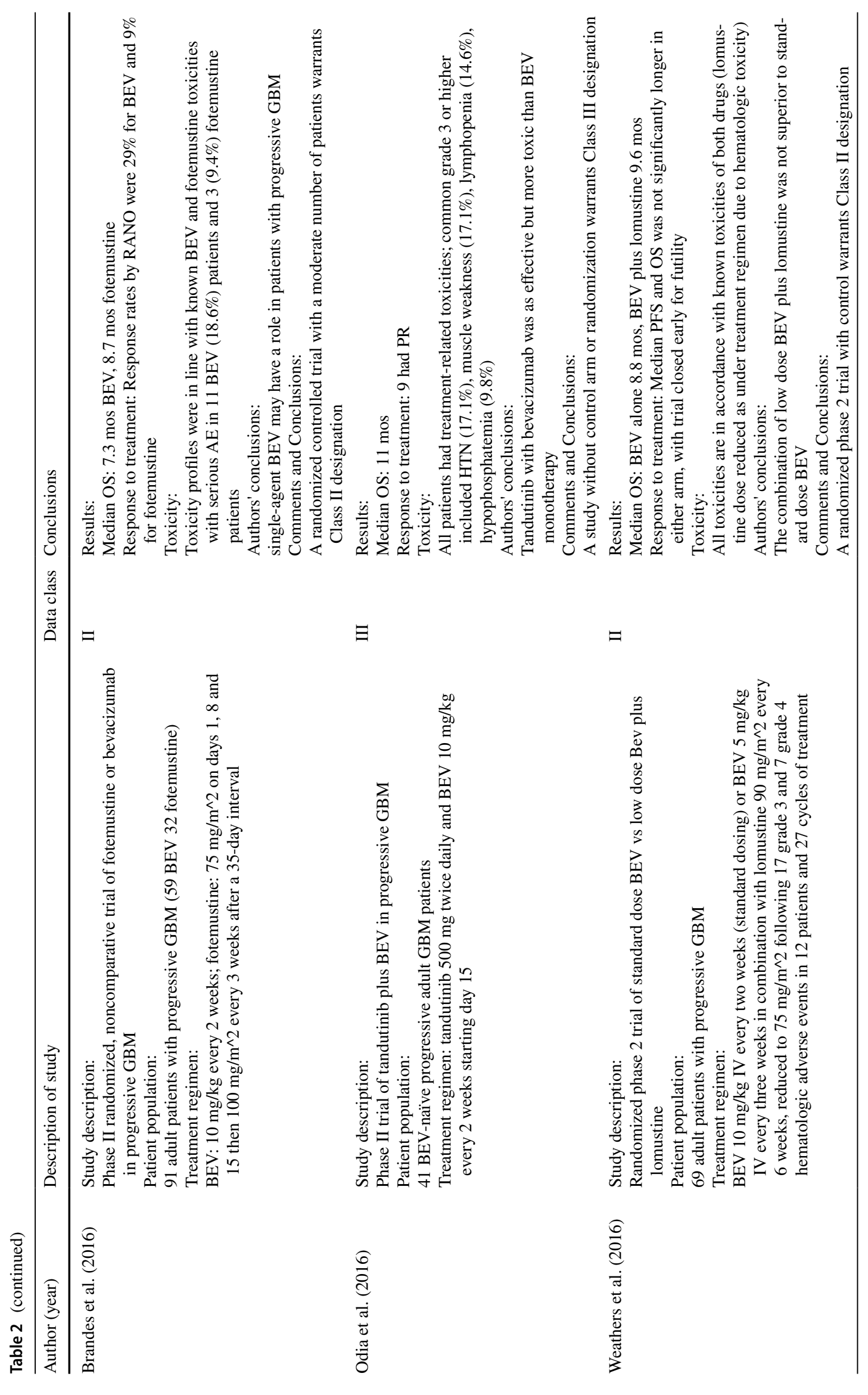




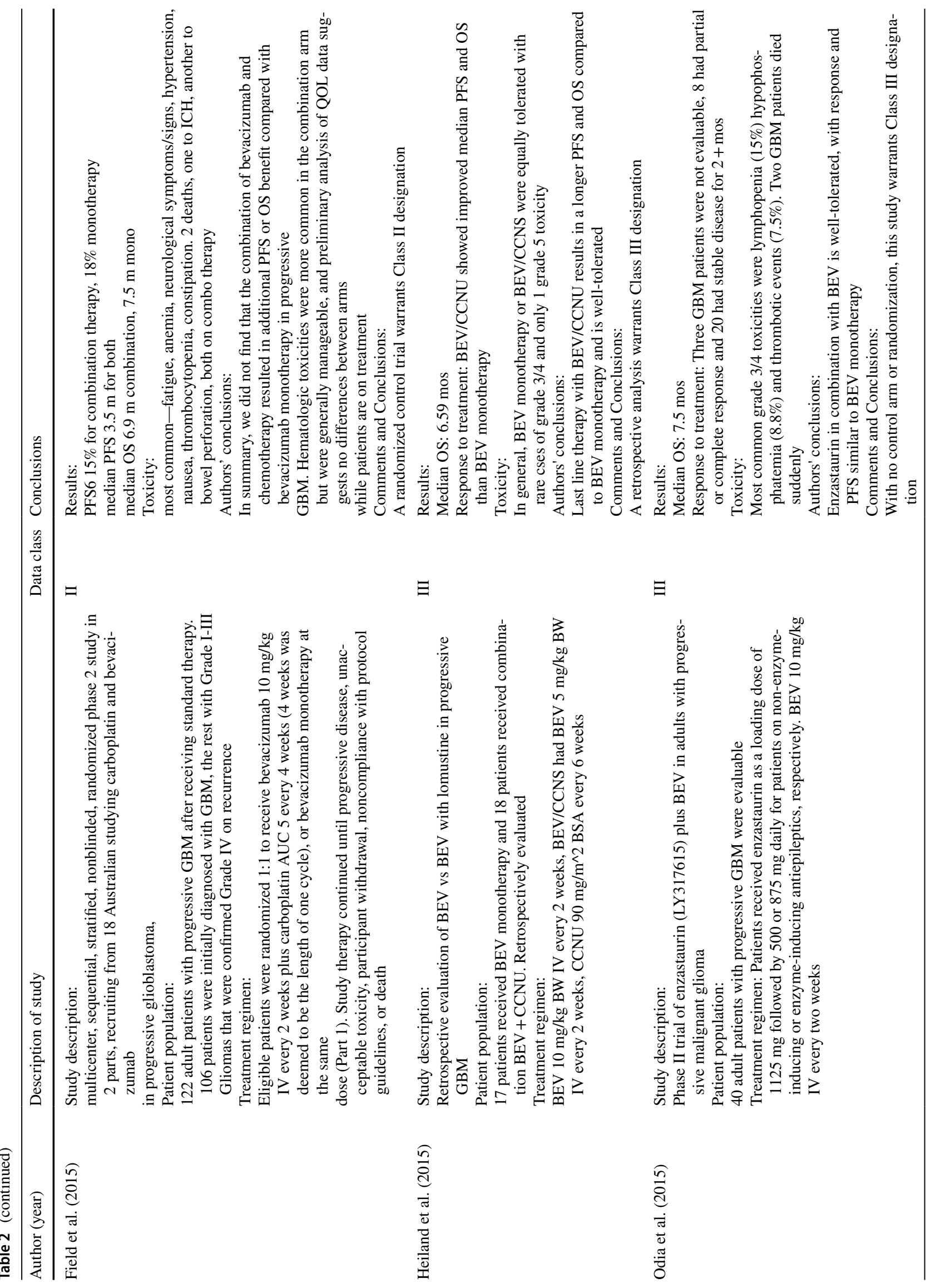




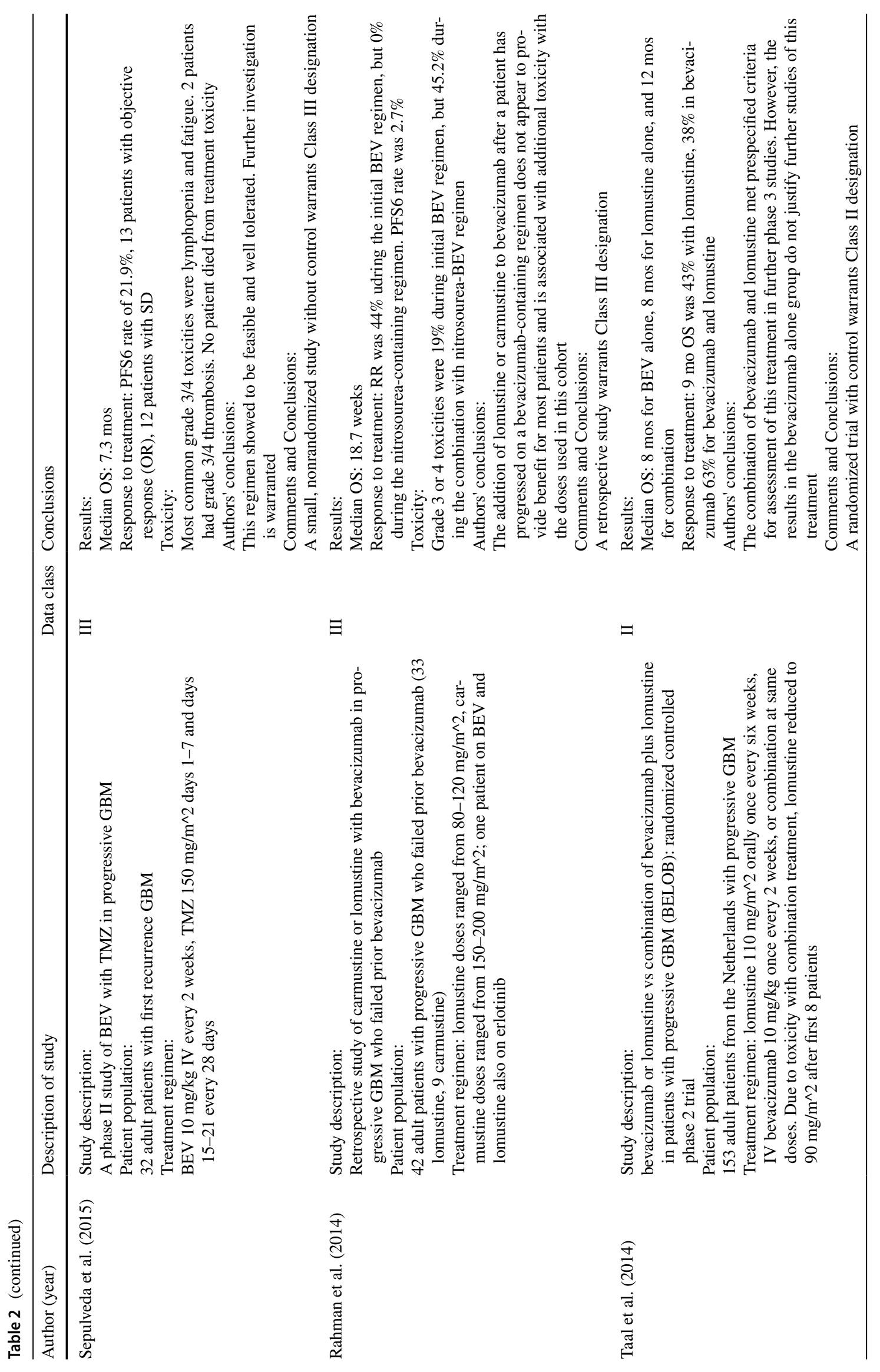




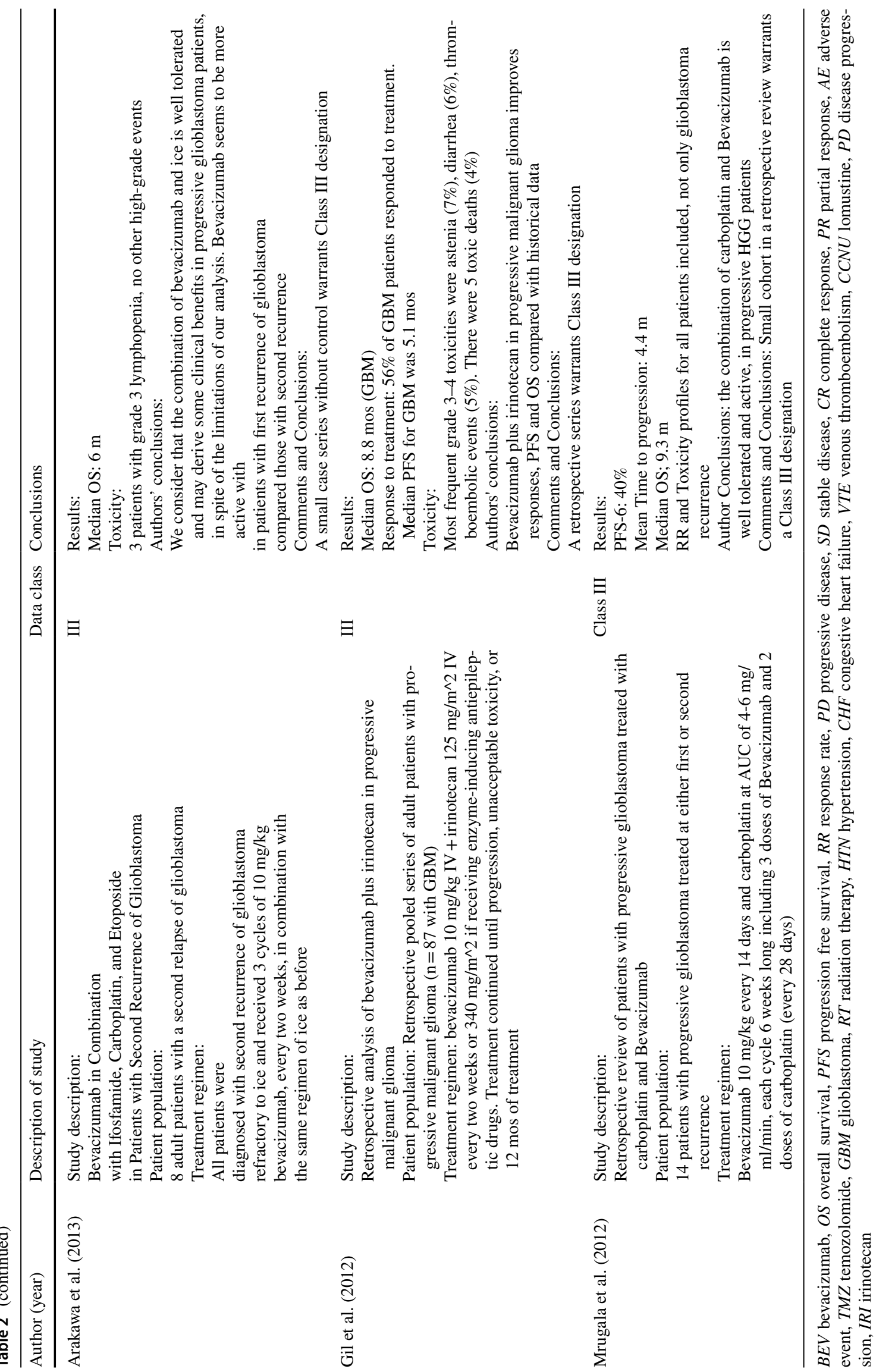


PFS and OS in the BEV + CCNU arm, yielding a conclusion that combination therapy as a last line therapy resulted in an improved PFS and OS compared to monotherapy. Two studies found results that showed no overall benefit. Jakobsen et al. evaluated Bev + Lomustine against BEV + irinotecan [34]. They found there was no significant difference in regards to survival between the two groups. Rahman et al. evaluated carmustine or lomustine with BEV after prior BEV failure and found there was no benefit in adding a nitrosourea agent to BEV treatment [38].

There were two studies designated Class III regarding combination therapy with fotemustine. Pasqualetti et al. studied BEV with fotemustine retrospectively with varying doses of both drugs which concluded there was no rationale to exclude BEV in combination therapy after failure of second line therapies [36]. In another study that came to a similar conclusion, Vaccaro et al. prospectively studied $\mathrm{BEV}$ with fotemustine in 13 patients [41]. Median OS was not reported in a fashion separable for only progressive glioblastoma patients, but RR showed no CR, 2 PR, 5 SD, with PFS of 3 months.

\section{ACE inhibitors and bevacizumab}

Gilbert et al. performed the only prospective study on ACE Inhibitors combined with BEV in this group since the previous guidelines. This was a randomized phase 2 trial of $\mathrm{BEV}$ and irinotecan vs BEV with TMZ in 117 patients [32]. Response rate for the TMZ arm was 3\% CR and 16\% PR and for the irinotecan arm was $4 \% \mathrm{CR}$ and $24 \% \mathrm{PR}$. The PFS6 for the TMZ group was 39\% and for the Irinotecan group was $38.6 \%$. The PFS6 surpassed the efficacy threshold in both arms regardless of prior TMZ sensitization. As this was a prospective, randomized trial, this warrants Class II designation.

Two retrospective studies involving irinotecan were performed. Johansen et al. retrospectively reviewed 26 patients treated with BEV and Irinotecan and found a trend toward improved PFS and OS, although toxicity and mOS were not reported [35]. They did notice a beneficial effect giving irinotecan and BEV together. Gil et al. completed another retrospective analysis of BEV with irinotecan in 87 patients pooling several series together [31]. Median OS was 8.8 months with Radiographic Response (RR) of $56 \%$ and a median PFS of 5.1 months. The authors concluded the combination therapy improved PFS and OS compared to historical data. These both warranted Class III designations as they were retrospective analyses.

\section{Synthesis}

The data from the prior guidelines found a PFS6 between 18.8 and $50.3 \%$ in the included studies. Based on the update of data, the PFS6 ranged from 2.7 to $53.8 \%$, with survival data somewhat skewed in some cases using mixed populations of patients at first or second recurrence and beyond. This ultimately didn't show any significant improvement in either mOS or PFS6. The highest level data came from a Class I designated Phase III study combining nitrosoureas with BEV and ultimately finding no significant survival advantage over monotherapy with BEV [43]. Further Class II data on the topic revealed similar findings and one was actually closed early for futility [27, 28, 42]. The data in general supported the utility of anti-angiogenic therapy throughout multiple courses of treatment for recurrence, however there was no reliable and discernible benefit in providing combination therapies with cytotoxic agents.

Question 3 In adults with progressive glioblastoma is the use of bevacizumab as a combination therapy with targeted agents superior to standard salvage cytotoxic chemotherapy as measured by progression free survival and overall survival?

\section{Prior recommendations}

In the previously published guidelines on the role of targeted therapies in the management of progressive glioblastoma in adults, bevacizumab combined with targeted therapies was reviewed and no specific recommendations were made based on a lack of strong evidence supporting a clearly defined benefit.

\section{Recommendation}

There is insufficient evidence to support a recommendation regarding this question.

\section{Study selection and characteristics of the updated search}

The initial search strategy included 3513 candidate articles. A total of 65 articles remained for full text review. From these, 3 articles were included in the final review for Question 3 and are included in Table 3 [45-47].

There was only one study showing a survival benefit with combination targeted therapy and bevacizumab. D'alessandris et al. performed a prospective trial evaluating erlotinib and BEV in combination vs BEV alone based on molecular profile of 10 patients with EGFRvIII expression or VEGF over expression [47]. Combination therapy showed a mOS 17 months and PFS6 of $100 \%$ while BEV monotherapy showed a PFS6 50\% and OS 6.75 months, at which point the authors concluded there was a significant survival benefit for combination therapy. As a small study lacking a control group, these were designated Class III data. 


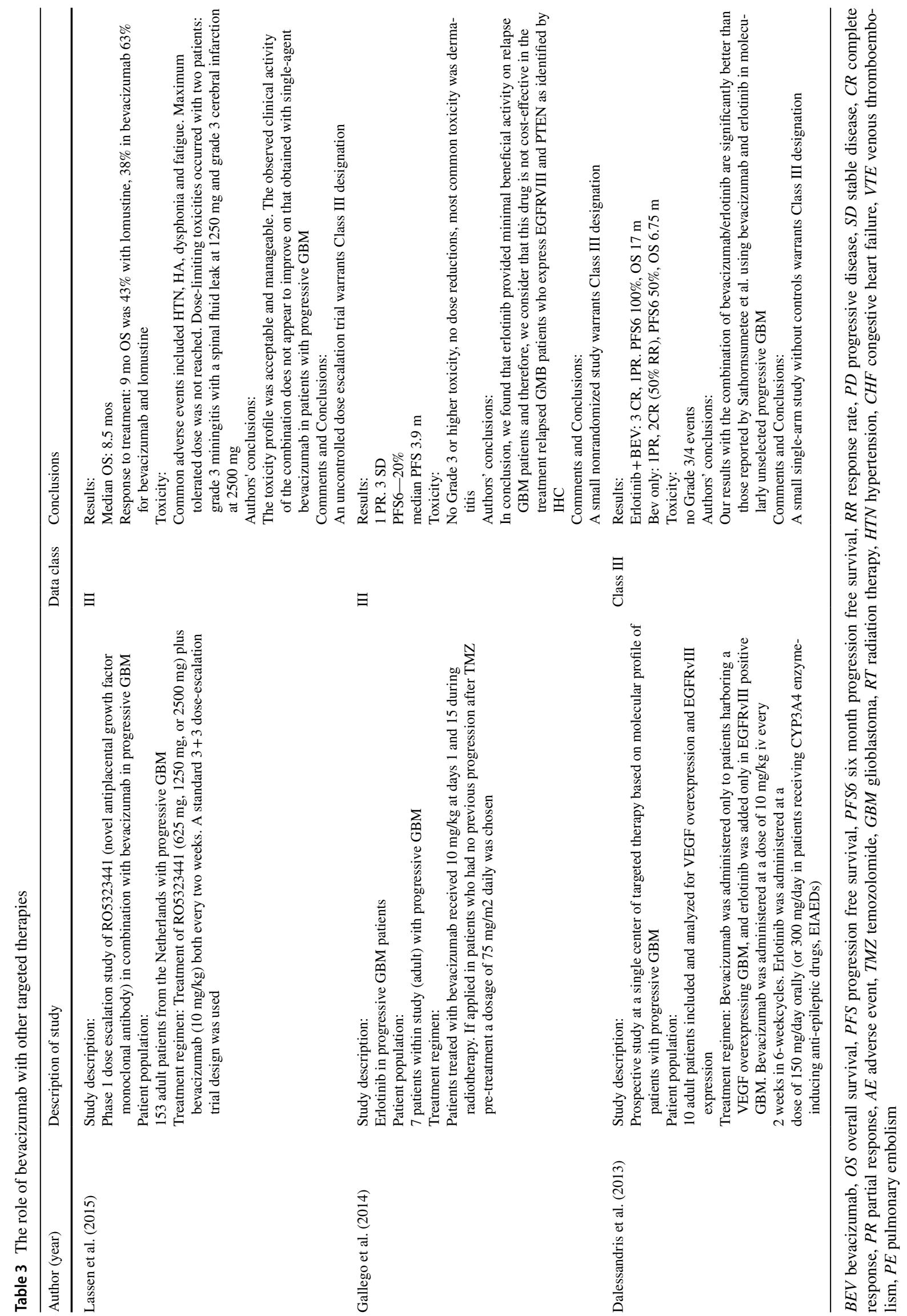


Lassen et al. evaluated a novel antiplacental growth factor monoclonal antibody (RO5323441) with BEV in 22 adult patients with progressive glioblastoma in a Phase I trial [46]. Median OS was 8.5 months with PFS 3.5 months in combination therapy, with the overall finding being that no significant improvement over BEV monotherapy was found in comparison to historical controls. Odia et al. studied enzastaurin in combination with BEV in 40 adult patients [45]. Median OS was 7.5 months. The same group then evaluated tandutinib with BEV in 41 patients, finding nonsuperiority and slightly more toxicity than BEV alone [48]. All three of these studies found nonsuperiority and due to a lack of control arms in the latter two studies and the first being a dose-escalation trial, they were all deemed Class III data.

\section{Synthesis}

In the prior guidelines published, only two trials were included that combined BEV with erlotinib or dasatinib, neither of which saw any PFS benefit, and both of which were not designated high enough class data to make a recommendation. The data reviewed since then include only Class III designations. Only one revealed significant survival benefit with erlotinib, albeit in a very small, highly specific study based on molecular profiling, while the rest showed only nonsuperiority. With the available studies and their limitations, there was insufficient high level data with consistent findings to make a treatment suggestion. This conclusion is not meant to suggest such combination studies are not worthy of further investigation and it is suggested that when available, and patients meet inclusion criteria, they be enrolled in properly designed studies of this issue.

Question 4 In adults with progressive glioblastoma is the use of targeted agents as monotherapy superior to standard salvage cytotoxic chemotherapy as measured by progression free survival and overall survival?

Prior Recommendations: In the previously published guidelines on the role of targeted therapies in the management of progressive glioblastoma in adults, targeted therapies other than BEV were reviewed and no specific recommendations were made based on a lack of strong evidence supporting a clearly defined benefit or detriment.

\section{Recommendation}

There is insufficient evidence to support a recommendation regarding this question.

\section{Study selection and characteristics of the updated search}

The initial search strategy included 3513 candidate articles. A total of 65 articles remained for full text review. From these, 23 articles were included in the final review for Question 4 and are included in Table 4 [49-71].

A total of 23 articles described the use of targeted therapies alone, not including BEV. [49-66, 68, 69, 72] Two studies used a targeted therapy in conjunction with repeat radiation therapy $[49,50]$. One study discussed the use of a cytotoxic agent (temsirolimus) in conjunction with Sorafenib [72]. Another study involved intracavity delivery of a targeted agent [59]. Retinoic acid naphthalene was given in another study, which was the only update discovered on the use of retinoids in progressive glioblastoma [61]. Combination of targeted agents (cediranib and cilengitide) were given in one study while cediranib and gefitinib were administered in combination in another study, providing the only two studies using combinations of targeted therapies [63, 67]. The remainder of studies were single agent targeted therapies [51-58, 60, 62, 64-69].

\section{Single agent targeted therapies}

Targeted therapies have been gaining attention recently, especially with the identification and characterization of several molecular variations among glioblastoma and the possibility of temozolomide resistance. Furthermore, there is a renewed vigor to find an effective, individualized molecular treatment for glioblastoma, especially in the progressive setting, beyond BEV.

Several small molecule targeted therapies were evaluated. Pitz et al. evaluated the use of PX-866 in 33 adult patients with progressive glioblastoma [52]. The study found a PFS6 of $17 \%$. The authors concluded that although the drug was well tolerated, it did not meet the predetermined efficacy end points. Aiken et al. provided a phase I clinical trial with dose escalation of a small molecule inhibitor of IGF1b in a small group of 7 patients [69]. They concluded there was a sustained clinical response. As it was a dose-escalation trial and had no control group, it was given a designation of Class III evidence. PLX3397, a Colony Stimulating Factor 1 Receptor inhibitor was given to 13 patients, finding a mOS of 9.5 months with no radiographic responses [66]. This was also designated Class III as it was a small cohort without randomization. Gilbert et al. studied cilengitide along with resection after recurrence in 26 patients and established the efficacy was only modest, yielding a Class III designation as a small Phase I trial in a small number of patients [71].

Depatux-m was studied by Gan et al. as a multicenter, Phase I dose escalation study, which combined newly diagnosed and progressive glioblastoma [64]. In the 


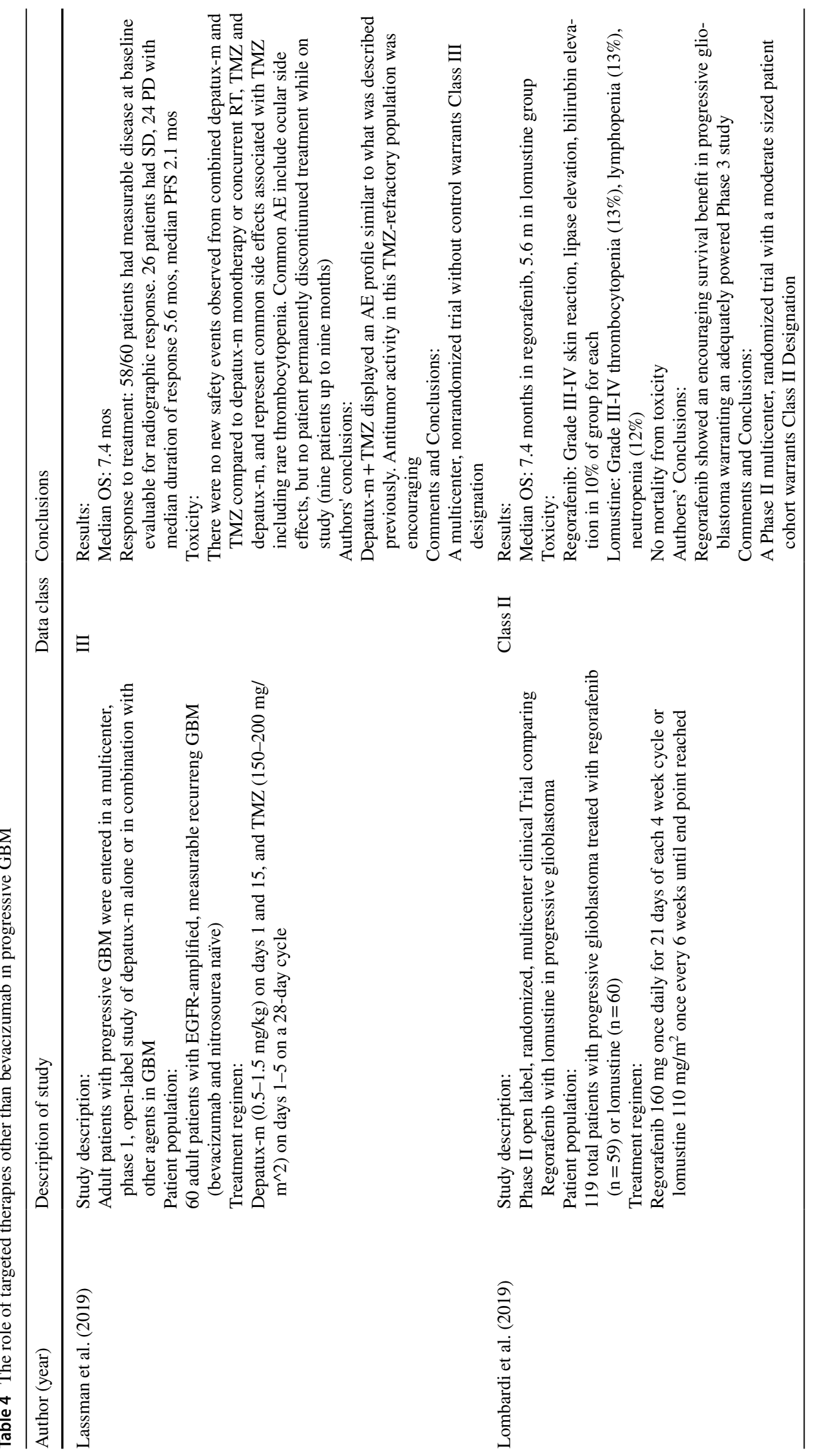




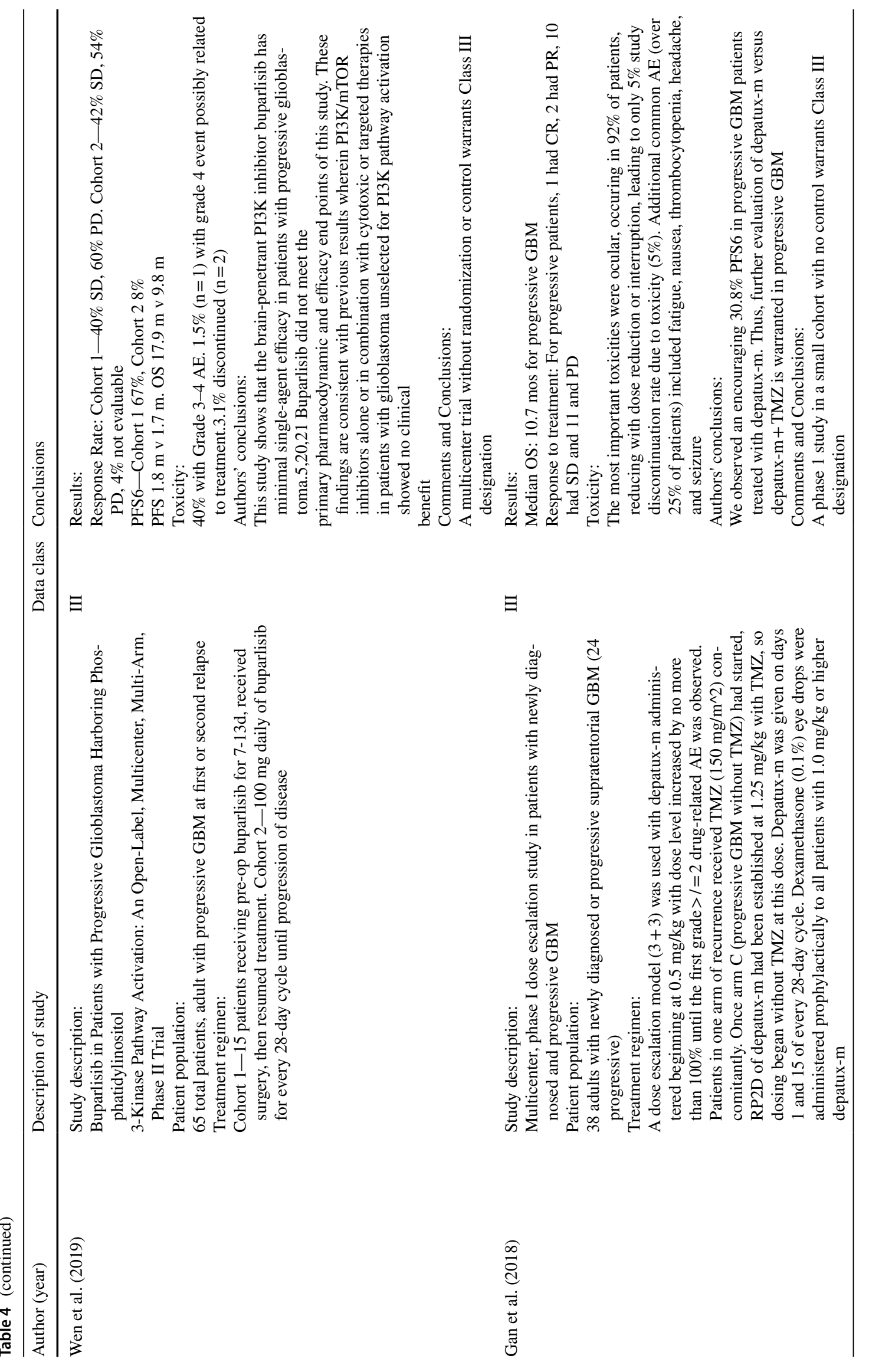




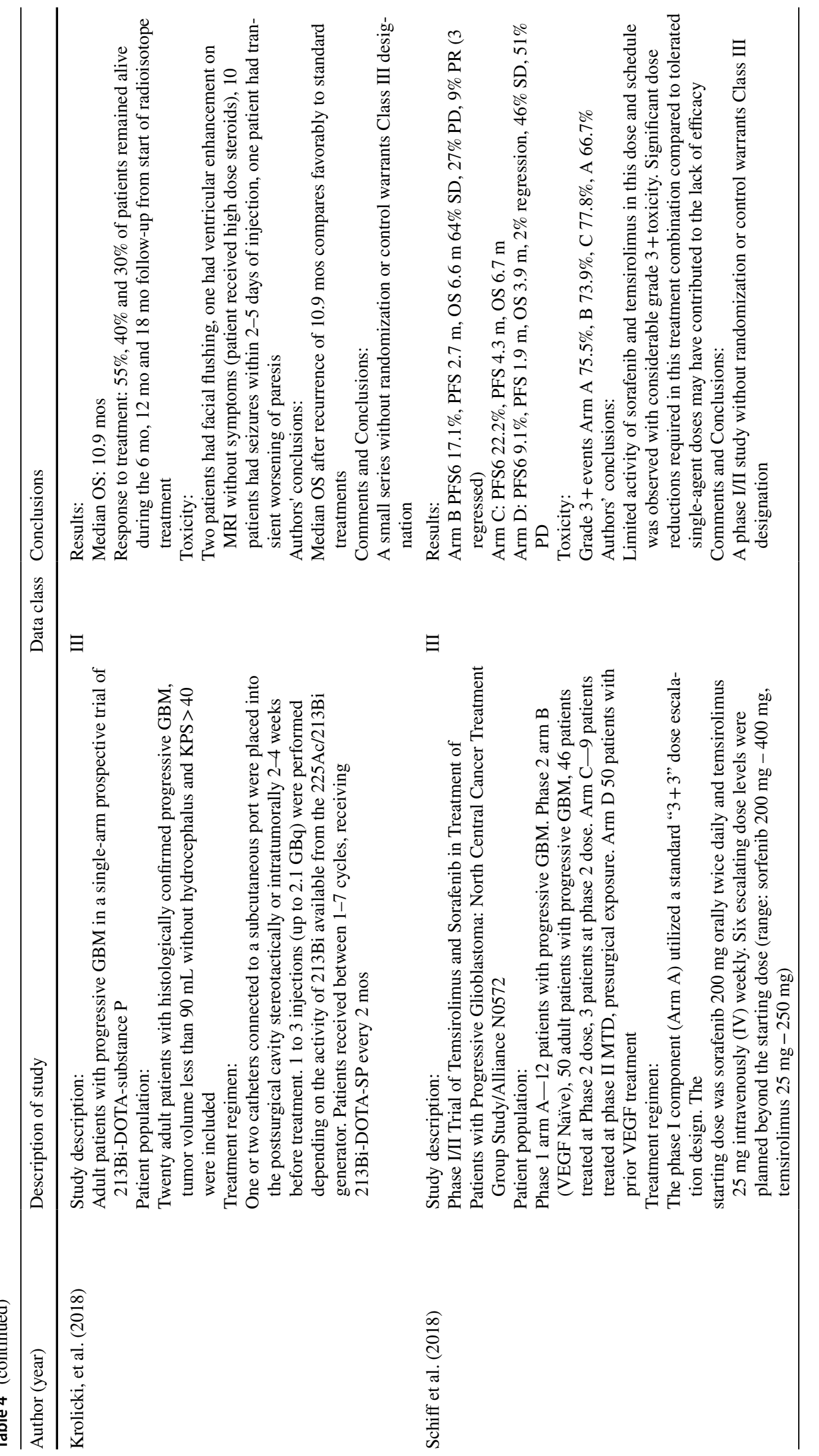




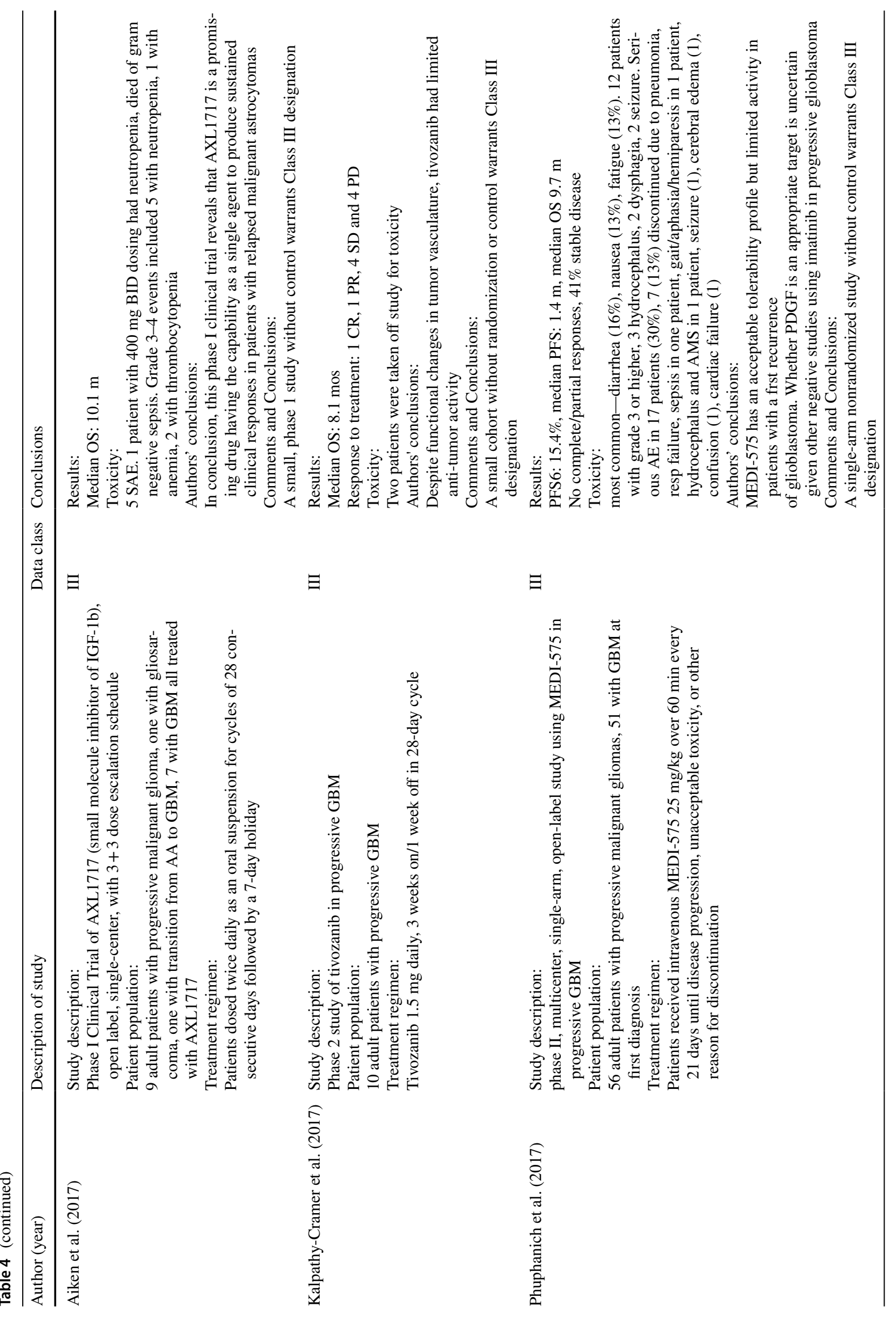




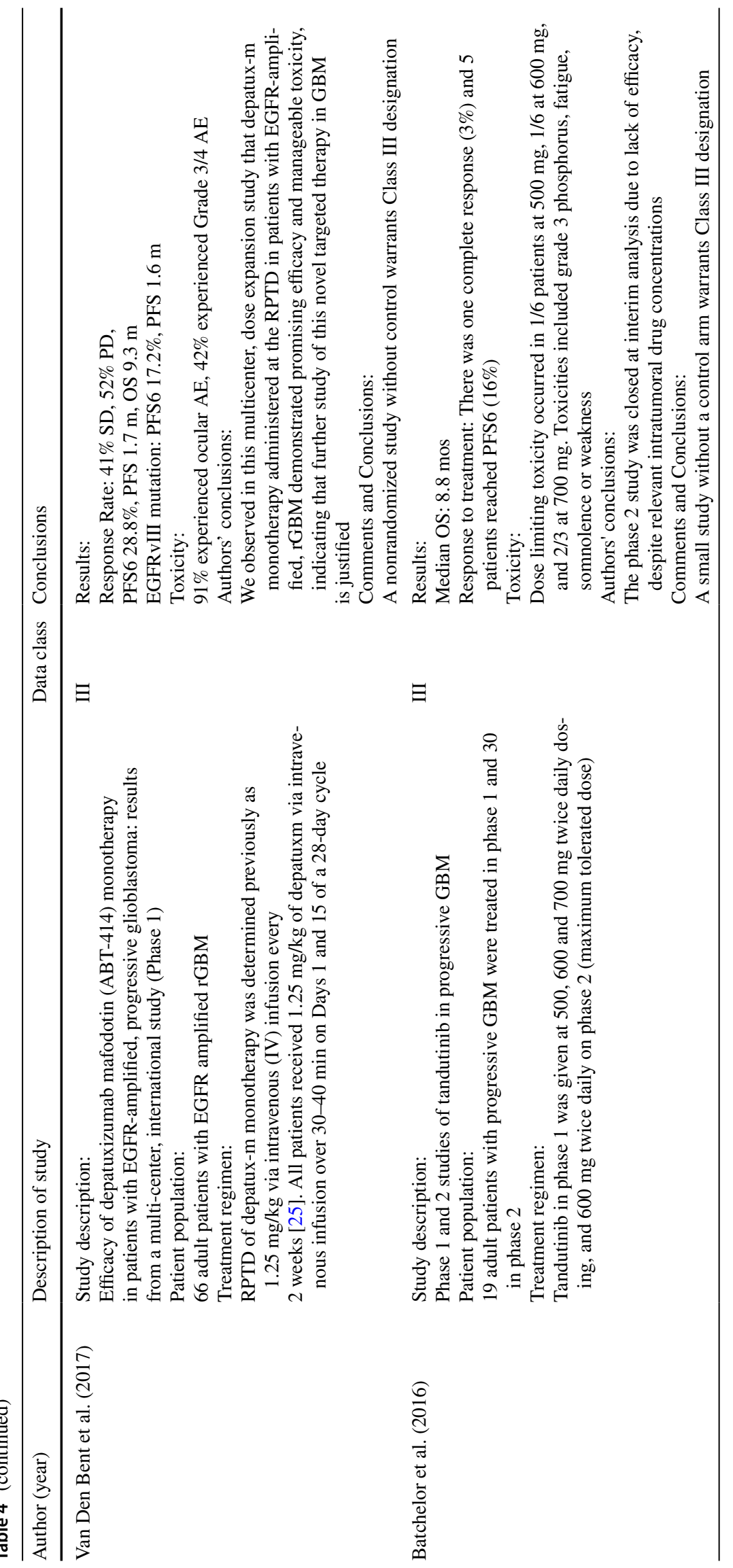




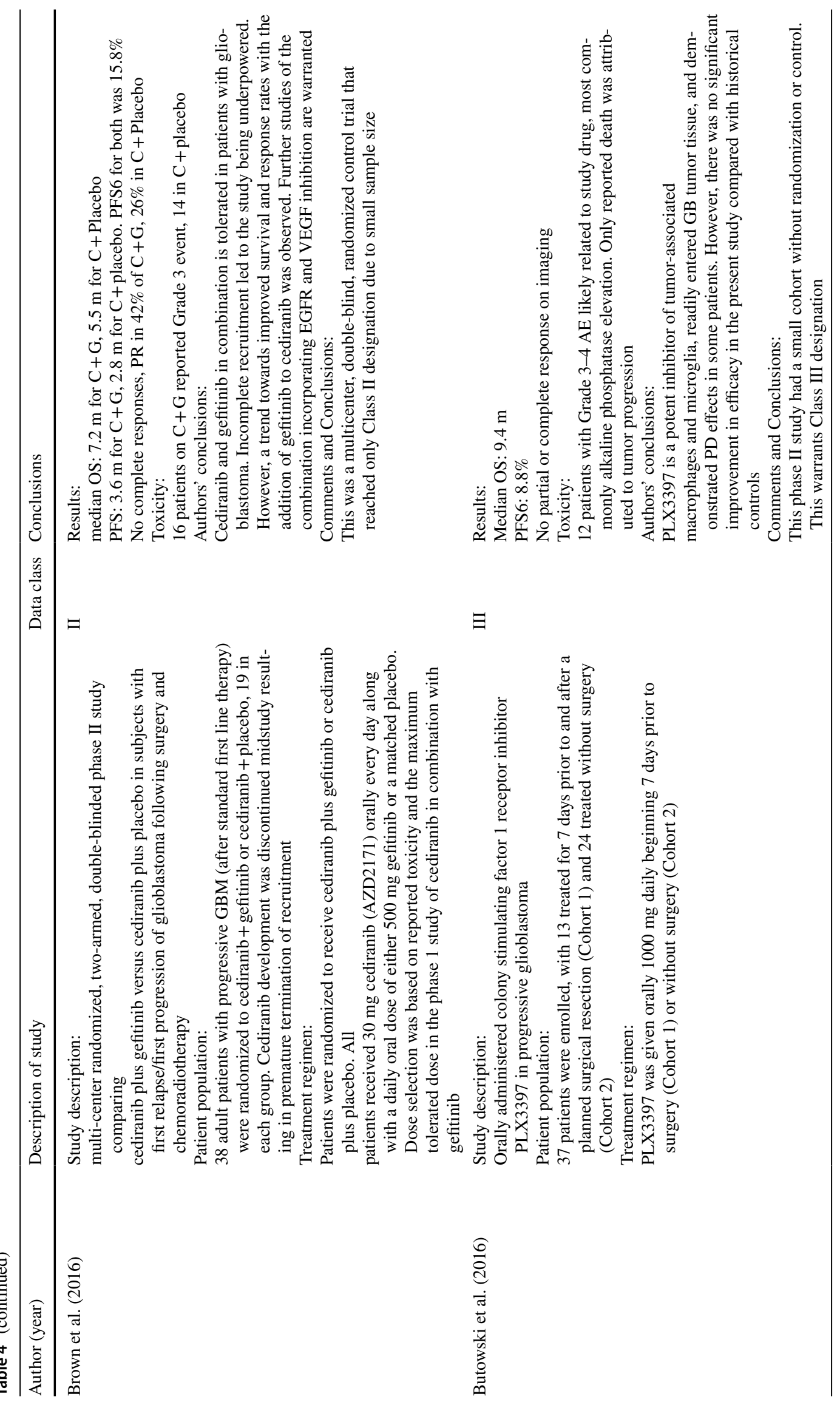




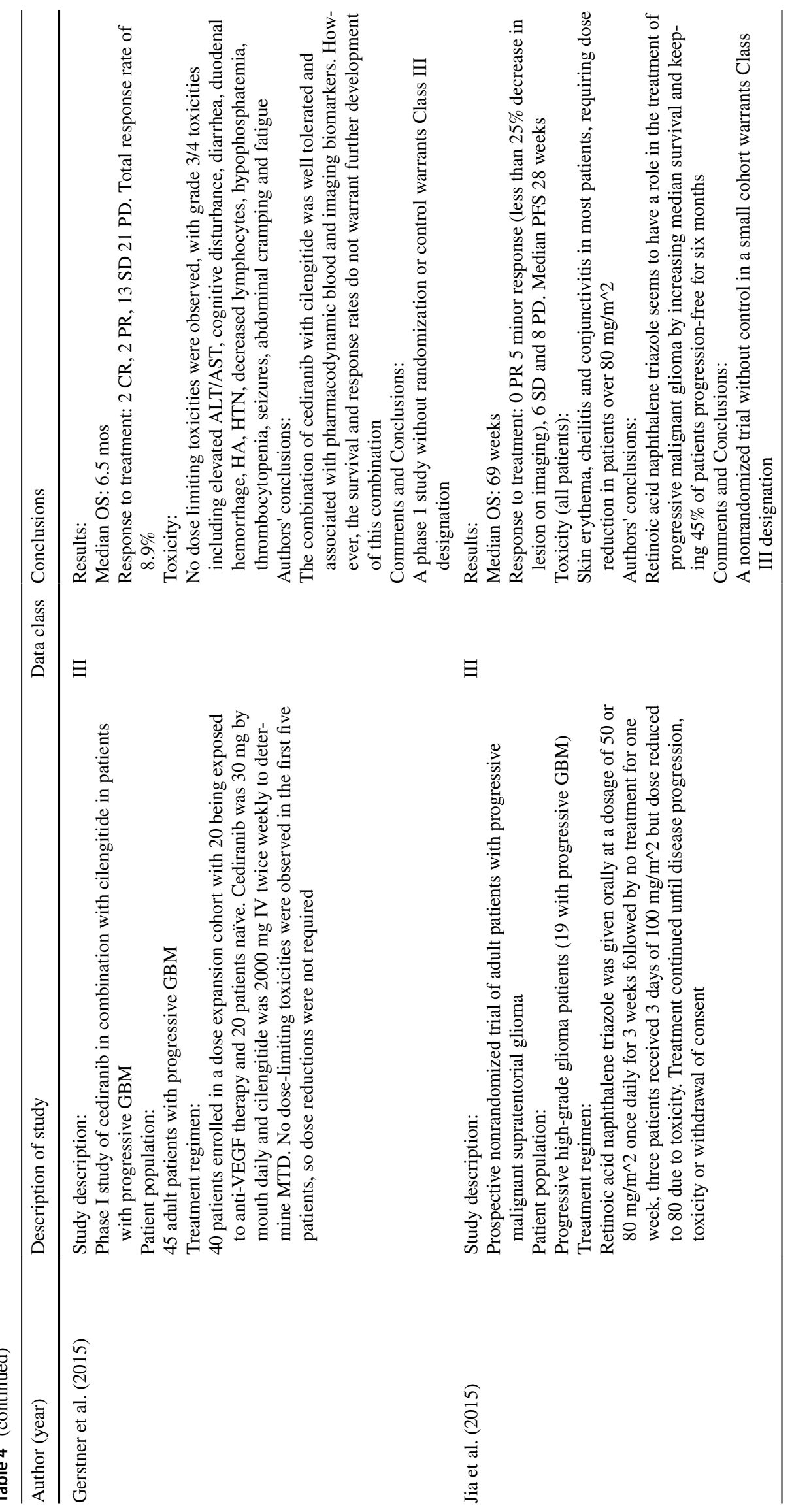




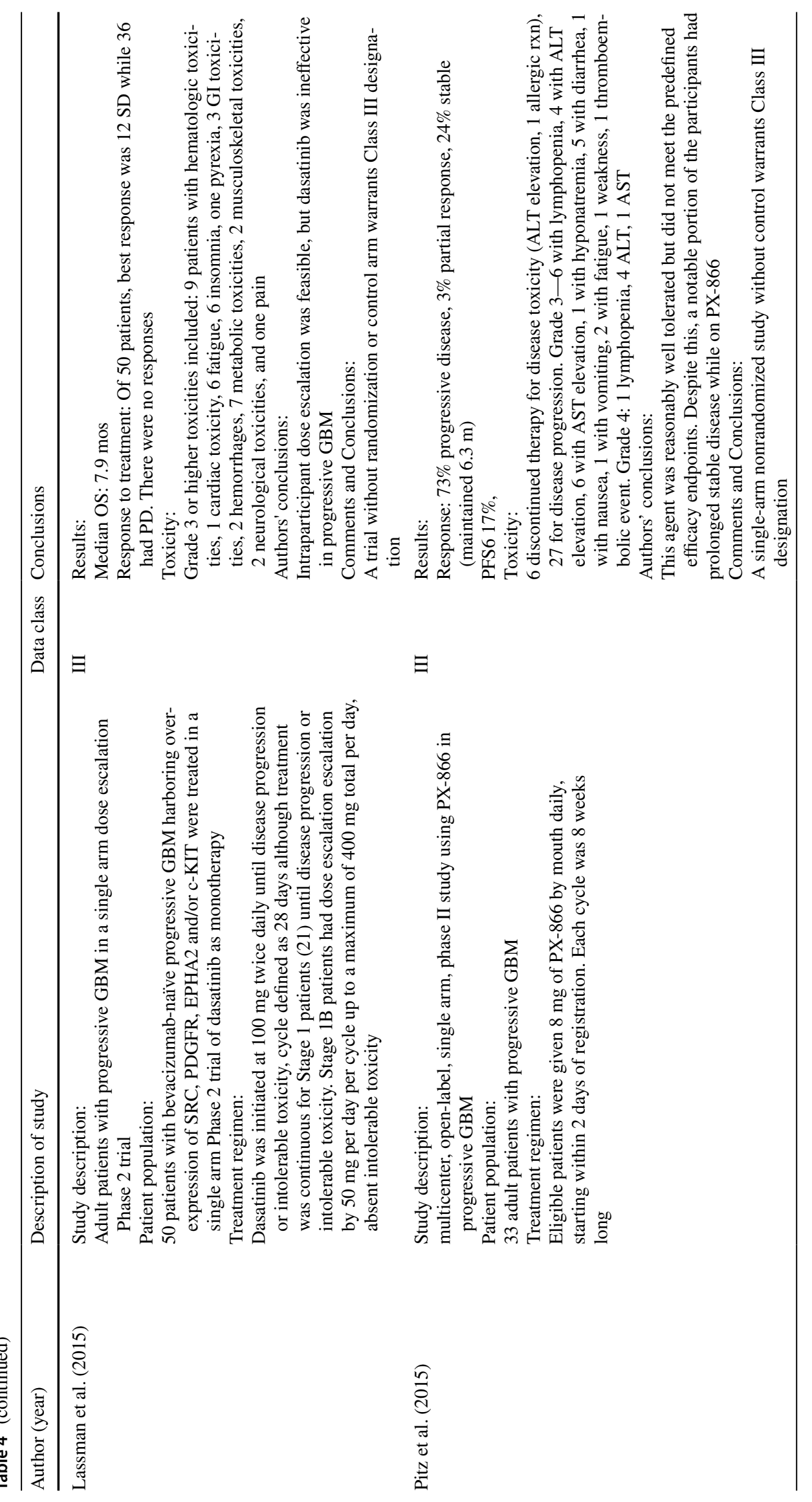




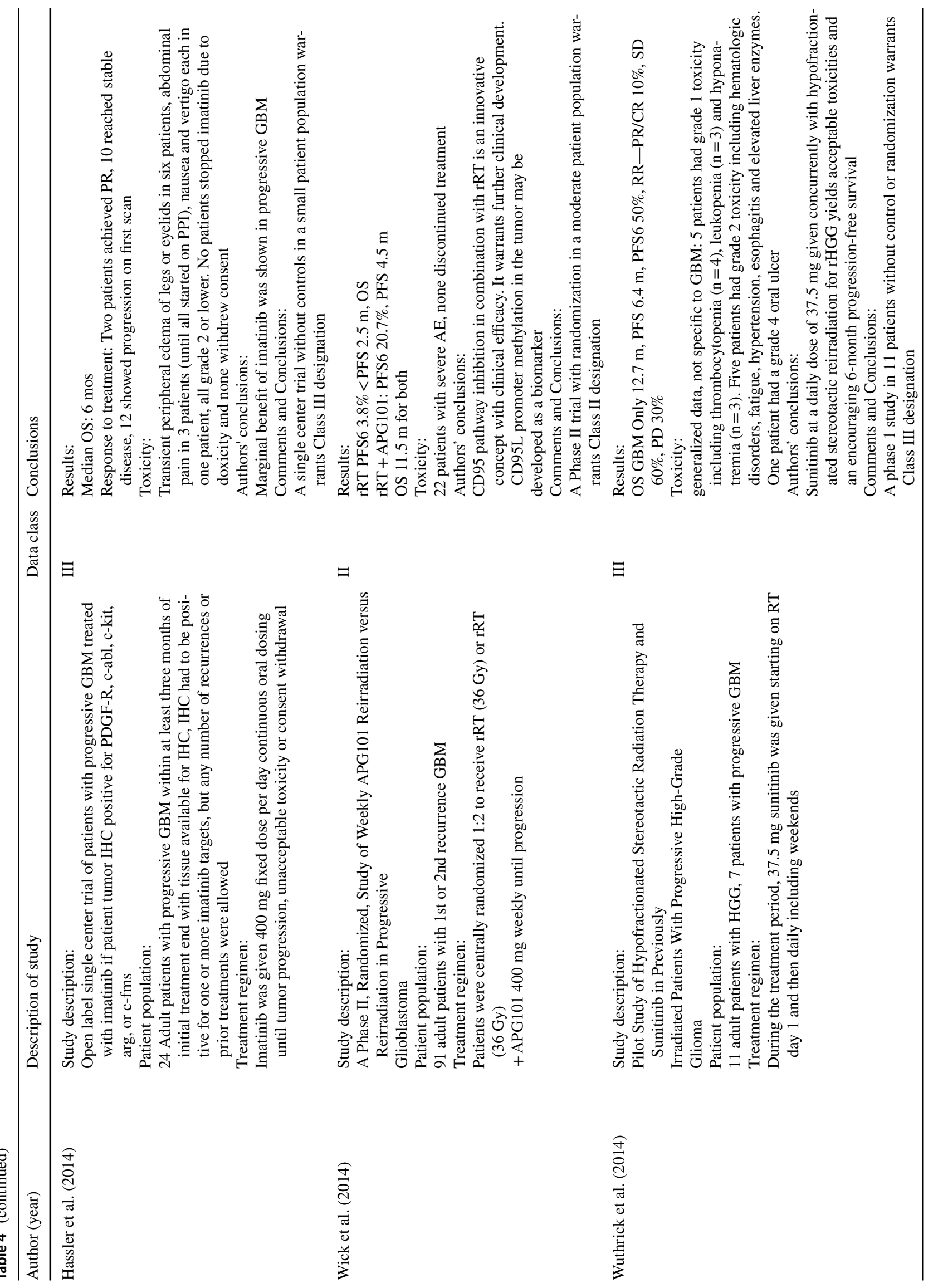




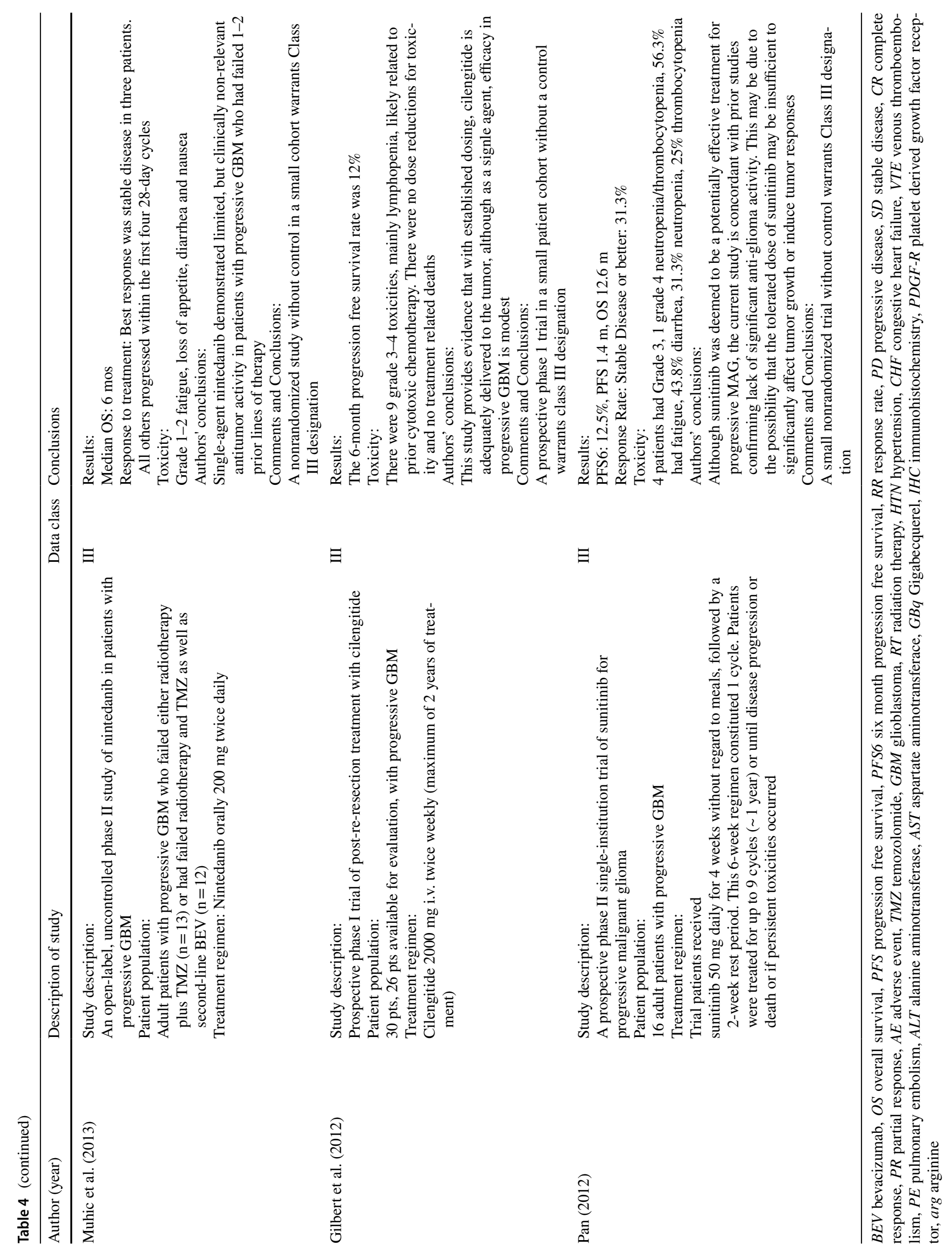


data separable for progressive patients, median OS was 10.7 months with $30.8 \%$ of patients reaching PFS6. Results were considered encouraging, the study was small without a control. Lassman et al. also studied administration of depatux-m in 60 patients with EGFR amplification in progressive glioblastoma without prior BEV or nitrosourea administration [57]. Median OS was 7.4 months and median PFS 2.1 months, with authors concluding the TMZ refractory population showed encouraging results. Van Den Bent et al. studied the efficacy of depatux-m in 66 patients [70]. All patients had EGFR amplified glioblastoma, and results showed a PFS6 of $28.7 \%$, PFS 1.7 months, mOS 9.3 months. The authors concluded that monotherapy in these patients showed some efficacy and tolerable toxicity requiring further study. These three trials lacked control groups and were nonrandomized, designating each as Class III evidence.

\section{Tyrosine kinase inhibitors}

Several studies also focused on tyrosine kinase inhibitors. Gallego et al. evaluated erlotinib in 13 progressive glioblastoma patients harboring PTEN, EGFR, and EGFRvIII overexpression [65]. PFS6 was 20\%. Authors felt this was of no benefit and that it was not cost-effective in this patient population. Tivozanib was evaluated in a phase II study of 10 patients by Kalpathy-Cramer et al. and showed a mOS of 8.1 months but discovered limited anti-tumor activity [60]. Lassman et al. evaluated 50 patients with BEV naïve recurrence, overexpression of SRC, PDGFR, EPHA2, and/or c-kit and administration of dasatinib monotherapy, also without any benefit [58].

Several of the included studies revolved around Tyrosine Kinase inhibitors with a focus on PDGFR interaction. Muhic et al. evaluated the use of nintedanib in 25 progressive glioblastoma patients with similar findings of a lack of anti-tumor activity [55]. Phuphanich reviewed the utility of targeting PDGFRalpha with MEDI-575 in a Phase II, multicenter, open-label study in 51 progressive glioblastoma patients [53]. PFS6 was $15.4 \%$ and mOS 9.7 months, but also showed limited activity in comparison to historical controls at first recurrence. Batchelor et al. completed Phase I and II trials of the PDGFRa inhibitor tandutinib in 19 patients and 30 patients, respectively [68]. Median OS was 8.8 months and PFS6 was $16 \%$ and the study was closed early due to a lack of efficacy. Hassler et al. studied the utilization of imatinib in 24 patients with PDGF-R, c-abl, c-kit, arg, or c-fms detected [62]. In a group that combined patients at varying numbers of recurrences, mOS was 6 months and only marginal benefit was seen.

Pan et al. attempted sunitinib administration in a phase II single institution trial of 16 patients [54]. $12.5 \%$ of patients reached PFS6, PFS was 1.4 months, and mOS was 12.6 months. $31.3 \%$ of patients had stable disease or better.
The authors concluded sunitinib could be a potentially effective treatment for progressive glioblastoma, although this and prior studies showed a lack of significant anti-glioma activity. This was designated Class III for a small, nonrandomized trial design without a control group.

Regorafenib, an oral receptor tyrosine kinase inhibitor targeting VEGFR-2, was compared with lomustine in a moderately sized, multi-center, randomized trial which showed an encouraging survival benefit with the use of regorafenib [56]. In about 119 patients trialed with progressive glioblastoma, the group given regorafenib saw mOS of 7.4 months compared to 5.6 months in the lomustine group. This study design warranted Class II designation.

\section{Retinoids}

Prior studies showed minimal to no activity of retinoids alone at recurrence, with a single example of a retinoid combined with TMZ showing a survival benefit compared to TMZ alone, but with only minimal radiographic response rates [73]. In our update, a study by Jia et al. was a prospective nonrandomized trial with administration of retinoic acid naphthalene in 19 patients [61]. Median OS was 69 weeks (16.1 months), Median PFS was 28 weeks and the authors concluded retinoic acid naphthalene triazole may have a role in the treatment of progressive glioblastoma with $45 \%$ of patients reaching PFS6.

\section{Stereotactic intratumoral delivery}

Krolicki et al. evaluated the efficacy of placement of a subcutaneous port with catheters stereotactically driven into the tumor cavity for future administration of 213Bi-DOTAsubstance $\mathrm{P}$ in a single arm prospective study [59]. Median OS was 10.9 months, $55 \%$ of patients remained alive at 6 months, $40 \%$ at 12 months, and 30\% were alive at 18 months of follow up from the start of treatment. The authors concluded the mOS of 10.9 months compared favorably to standard treatments. This was also designated Class III as the study was a small sample size without a control group.

\section{Buparlisib (PI3K inhibition)}

Wen et al. used buparlisib to target phosphatidylinositol3 -kinase in 65 patients with progressive glioblastoma at their first or second relapse [51]. Results showed PFS6 of $67 \%$ in cohort $1,8 \%$ in cohort 2 . PFS was 1.8 months in Cohort 1 and 1.7 months in cohort 2 with OS of 17.9 versus 9.8 months, respectively. The authors concluded the PI3K inhibitor, known to penetrate the blood brain barrier, had efficacy as a single agent but did not meet pharmacodynamic 
and efficacy end points and the findings were consistent with prior studies showing no clinical benefit in patients unselected for PI3K pathway activation. This was designated Class III as the trial lacked randomization and there was no control group.

\section{Dual agent targeted therapy}

We encountered two studies using a combination of targeted therapies [63, 67] One, a study by Gerstner et al., gave cilengitide, an anti-angiogenic small molecule targeting integrins, and cediranib, an oral VEGF inhibitor, in a phase I trial, warranting class III designation [63]. Forty patients were treated and results showed a mOS of 6.5 months and response rates at $8.9 \%$, with a conclusion that although the regimen was well tolerated, there was no overall benefit discerned to warrant further study. Brown et al. compared cediranib and gefitinib to cediranib and placebo with 19 patients in each group [67]. Response rates were $42 \%$ PR in the combination group and $26 \%$ in the placebo arm and mOS of 7.2 months in the combination group and 5.5 months in the placebo arm. PFS was 3.6 months in the combination therapy group and 2.8 months in the placebo group. The study suffered from incomplete recruitment due to discontinuation of cediranib production mid-study, but concluded there was a trend toward improved survival and response rate. This was designated Class II data as it was a multicenter, double-blind, randomized, control trial with a small sample size.

\section{Radiation and targeted therapies}

We found two studies regarding targeted therapies specifically and intentionally used in combination with repeat radiation therapy $[49,50]$. Wuthrick et al. completed a pilot study of hypofractionated stereotactic radiation therapy in conjunction with sunitinib [49]. Eleven patients were studied, 7 of which were progressive glioblastoma previously treated with radiotherapy. Results specifically for progressive glioblastoma patients included an OS of 12.7 months, PFS of 6.4 months, PFS6 of 50\%, and response rates including $10 \%$ PR/CR, $60 \%$ with SD and $30 \%$ with PD. The authors concluded the results were encouraging in terms of PFS6 and safety and warrant further study. This was designated Class III for a small, nonrandomized cohort without a control group.

Wick et al. studied the effect of APG101 (CD95/CD95L inhibitor) with reirradiation ( $\mathrm{rRT}$ ) versus only reirradiation [50]. 91 patients at the first or second recurrence were randomized and the irradiation only group had a PFS6 of 3.8\% and PFS 2.5 months, while the combination treatment group had a PFS6 of $20.7 \%$ and PFS 4.5 months. They concluded CD95 pathway inhibition with rRT had clinical efficacy and needed further study and potentially more identification of patients with CD95L promoter methylation as a biomarker. This was designated Class II evidence with a moderate size population and randomization.

\section{Synthesis}

The prior guidelines on targeted therapy included studies on a wide array of targeted therapies. The only new studies providing encouraging results but without significant discernible benefit were the studies regarding Depatux-m, showing mOS of 7.4 to 10.7 months in early phase trials, and radiation with targeted therapies with one Class II study showing a significant benefit compared to RT alone at recurrence. Another promising Class II study showed only a trend toward a survival benefit when combining Gefitinib and cediranib, improving slightly upon the data in the prior guidelines showing a gefitinib with at most a potential efficacy in monotherapy. Regorafenib, another set of class II data, saw a survival benefit compared to cytotoxic treatment. The rest of the evidence was Class III evidence with significant limitations in trial and study designs, with no new data showing improved clinical benefit with tyrosine kinase inhibitors, buparlisib, or retinoids. Thus, there was no significant evidence to provide a clear recommendation at this time. This conclusion is not meant to suggest that studies of targeted agents alone or in combination are not worthy of further investigation and it is suggested that when available, and patients meet inclusion criteria, they be enrolled in properly designed studies that address these modalities.

Question 5 In adults with progressive glioblastoma is the use of targeted agents in combination with cytotoxic therapies superior to standard salvage cytotoxic chemotherapy as measured by progression free survival and overall survival?

Prior Recommendations: In the previously published guidelines on the role of targeted therapies in management of progressive glioblastoma in adults, targeted therapies other than bevacizumab in combination with cytotoxic therapies were reviewed and no specific recommendations were made based on a lack of strong evidence supporting a clearly defined benefit or detriment.

\section{Recommendation}

There is insufficient evidence to support a recommendation regarding this question. 


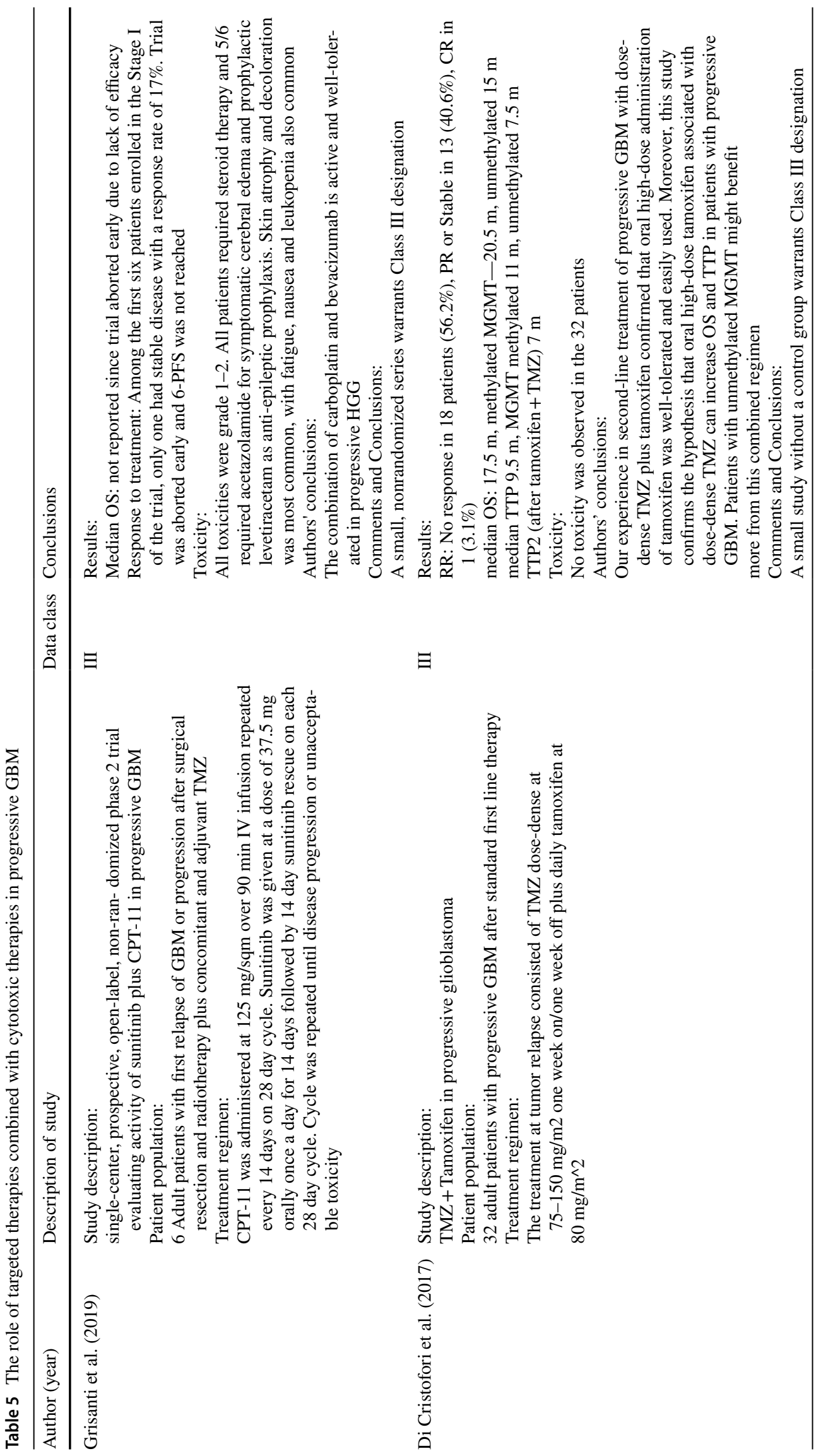




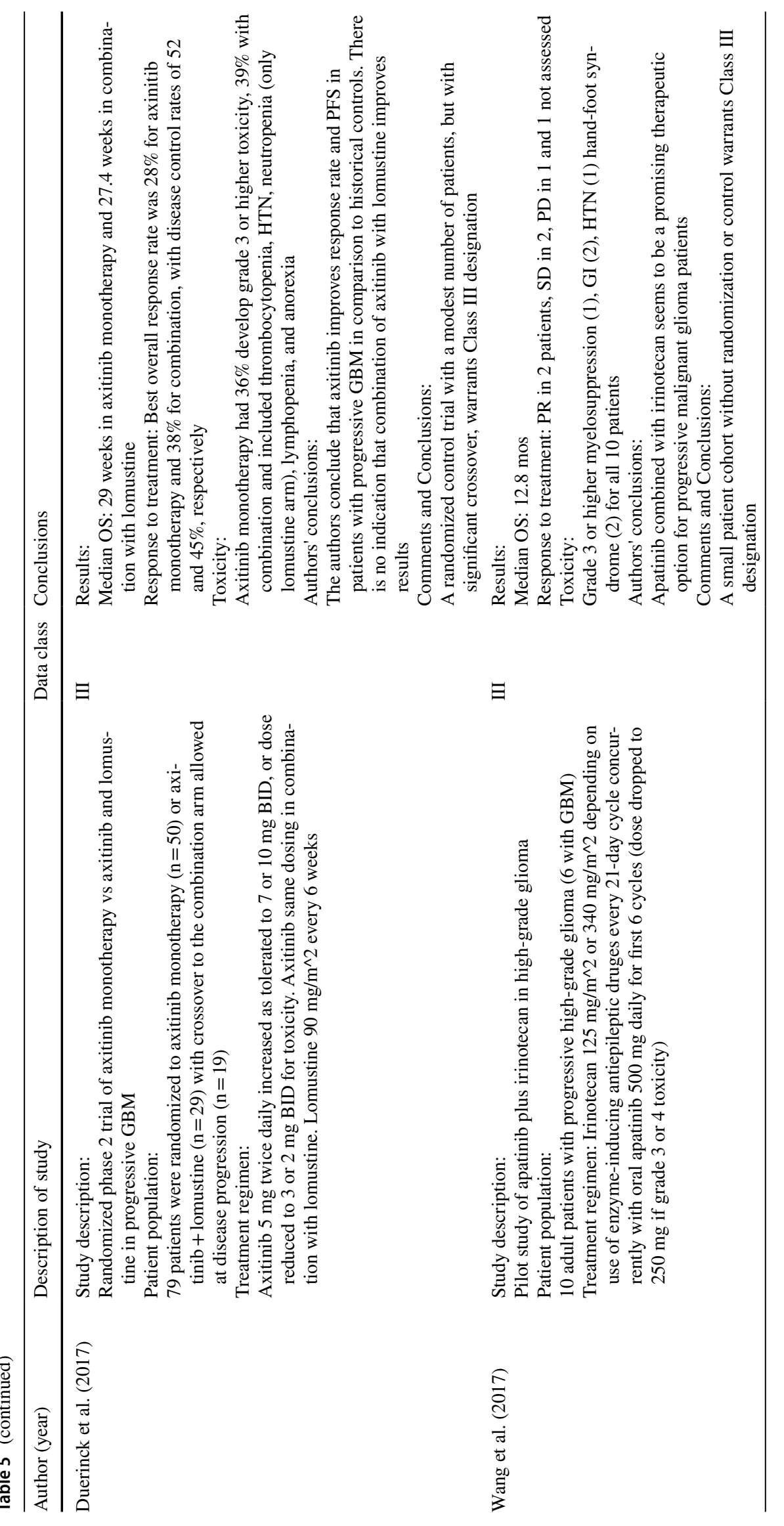




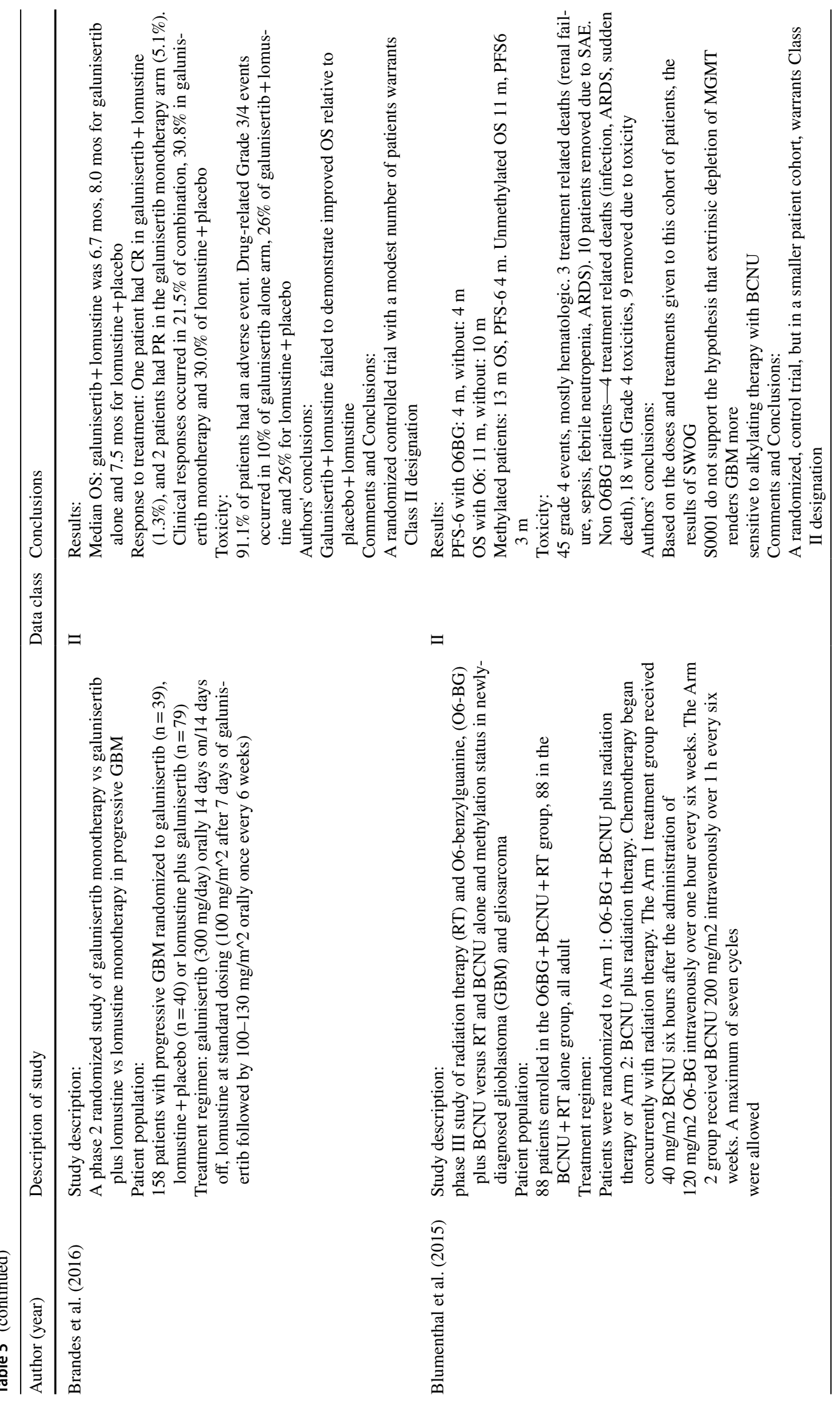




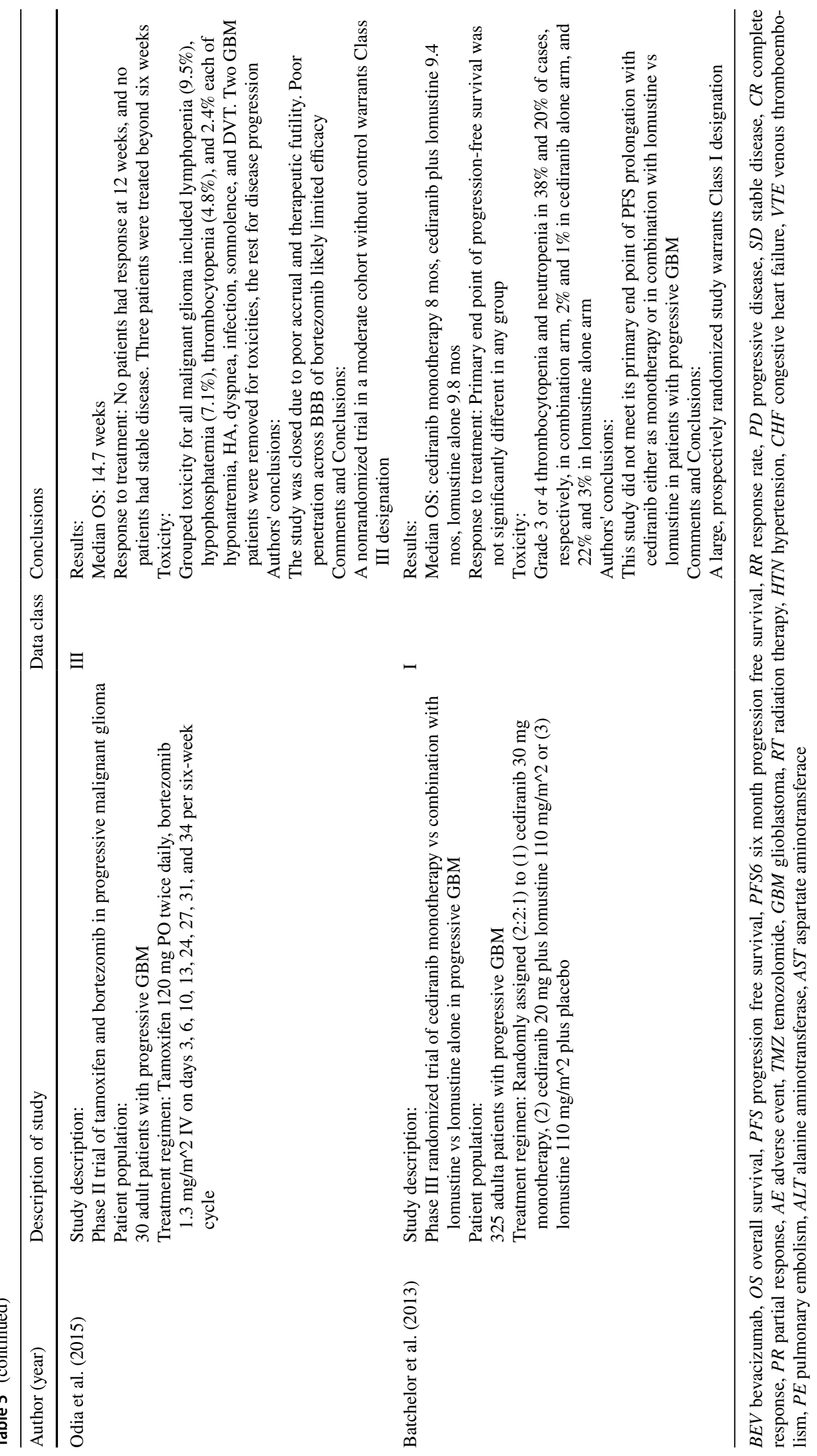




\section{Study selection and characteristics of the updated search}

The initial search strategy included 3513 candidate articles. A total of 65 articles remained for full text review. From these, 8 articles were included in the final review for Question 5 and are included in Table 5 [74-81].

A total of 8 papers were reviewed describing combination therapy with targeted therapy and cytotoxic therapy [74-81]. Two studies included the use of tamoxifen [75, 81]. Four of the studies included the use of nitrosoureas like lomustine and BCNU [77-80]. The remainder of studies evaluated the use of single agent targeted therapies with other cytotoxic chemotherapies $[72,74,76]$.

\section{Tamoxifen}

Tamoxifen is a selective estrogen-receptor modulator that has been shown to have some effect in glioblastoma patients [82-84]. Di Cristofori et al. studied 32 adult patients receiving TMZ and tamoxifen [81]. Median OS was 17.5 months, with methylated MGMT patients showing a 20.5 month mOS and 15 months in unmethylated patients. Methylated patients had a time to progression interval of 11 months and 7.5 months in unmethylated patients, with a median time to progression of 9.5 months. The second time to progression (after TMZ + tamoxifen) was another 7 months. Authors concluded there was a benefit to the dual treatment regimen in both patient groups. This was designated Class III evidence as the study was small and without a control group. Next, Odia et al. combined tamoxifen with bortezomib (inhibits the mammalian 26S proteasome involved in the ubiquitin-proteasome pathway) in 30 patients [75]. They closed their study early and deemed bortezomib to be of limited efficacy due to poor crossing of the blood brain barrier, with minimal comment on tamoxifen. This was designated Class III as well with lack of a control group and a nonrandomized design.

\section{Nitrosoureas}

Batchelor et al. performed a phase III randomized trial using cediranib monotherapy, cediranib and lomustine, lomustine or lomustine alone in 325 patients [80]. The authors concluded the study did not produce a prolongation of mOS PFS either in combination therapies or as monotherapy of either lomustine or cediranib. As it was a large, prospective, randomized study, this warranted Class I designation. Blumenthal et al. performed a phase III study of RT with O6-benzylguanine with BCNU versus radiotherapy and BCNU alone with comparison to methylation status [79]. Results in 88 patients showed no significant difference between the treatment arms and methylation status. They concluded that extrinsic depletion of MGMT does not necessarily make glioblastoma more susceptible to BCNU alkylation therapy. The moderate size and randomized, control trial design portended a Class II designation.

Brandes et al. performed a phase II randomized study evaluating galunisertib (TGF-b inhibitor) monotherapy vs combination therapy with lomustine versus lomustine monotherapy in 158 patients [78]. mOS was 6.7 months in the combination group, 8 months with galunisertib monotherapy, and 7.5 months in lomustine with placebo. The authors concluded that combination therapy failed to show any overall improvement of OS relative to the placebo plus lomustine group. This warranted Class II designation for the randomized, control trial design with a moderate sized patient cohort. Duerinck et al. evaluated axitinib (a small molecule tyrosine kinase inhibitor) monotherapy in comparison to combination with lomustine in 79 patients [77]. The authors concluded that axitinib improved response rate but there was no overall benefit as combination therapy, with the small case series design designating the data Class III.

\section{Kinase inhibitors}

Grisanti et al. evaluated irinotecan in conjunction with sunitinib [76]. They evaluated a small number of patients $(n=6)$ and never reached their PFS6 endpoint before terminating the trial early, generating Class III data. Schiff et al. evaluated temsirolimus with sorafenib (protein kinase inhibitor with activity against VEGFR, PDGFR, RAF kinases) [72]. The study found toxicity prominent among all arms of the trial requiring dose reductions, resulting in a lack of efficacy compared to single agent administration. The combined Phase I and II trial was without randomization and there was no control group, generating a Class III designation.

Wang et al. evaluated irinotecan with apatinib (tyrosine kinase inhibitor selective for VEGFR2) in 10 patients, 6 of which had glioblastoma [74]. The conclusion was that the combination may be promising, but results were limited due to a small patient cohort without randomization or control group, giving it Class III designation.

\section{Synthesis}

In the prior guidelines, there were mixed reviews regarding combined targeted and cytotoxic therapy regimens, without any studies showing reproducible proof of positive PFS or OS benefit. In this update, tamoxifen studies yielded Class III data and no overall benefit. In the data for nitrosoureas, all the Class I and Class II evidence available revealed there was no benefit beyond monotherapy with a targeted agent and at times with the nitrosourea alone, thus not identifying any synergistic effect. The same held true for the kinase inhibitors combined with cytotoxic agents where all data was 
Class III showing no overall efficacy beyond monotherapies. This did not provide sufficient evidence to make a recommendation regarding targeted therapies in combination with cytotoxic agents. This conclusion is not meant to suggest such combination studies are not worthy of further investigation and it is suggested that when available, and patients meet inclusion criteria, they be enrolled in properly designed studies of targeted agents combined with cytotoxic agents.

Question 6 In adults with progressive glioblastoma is the use of immunotherapy alone superior to standard salvage cytotoxic chemotherapy as measured by progression free survival and overall survival?

Prior Recommendations: In the previously published guidelines on the role of targeted therapies in the management of progressive glioblastoma in adults, immunotherapeutic agents were not reviewed.

\section{Recommendation}

There is insufficient evidence to support a recommendation regarding this question.

\section{Study selection and characteristics of the updated search}

The initial search strategy included 904 candidate articles. A total of 31 articles qualified for full text review. From these, 26 articles met eligibility criteria and were included in the final review for Question 6 and are included in Table 6 [71, 85-111].

\section{T-Cell therapies}

Ahmed et al. performed a phase I open label dose escalation trial using HER2-specific CAR-T cells in 17 total patients, 10 of which were adult patients. Results showed PFS of 3.5 months and mOS 11.1 months after infusion, 24.5 months after initial diagnosis. They concluded the HER2-CAR virus specific T cells (VSTs) are safe and may portend a clinical benefit [85]. In another specific T-Cell study, Schuessler et al. reported on autologous CMV specific $\mathrm{T}$ cell administration in a study including 10 patients with complete data. Median OS of those with at least one infusion was 13.4 months and concluded the therapy was safe with a potential long-term clinical benefit [105]. Tsuboi et al. studied autologous tumor specific T-lymphocytes (ATTLs) and suggested efficacy however with a potentially only temporary antitumor effect [110]. As phase I trials with small patient populations, these were all designated Class III.

\section{Vaccines}

Two studies included revolved around the use of dendritic cell (DC) vaccination. Chang et al. prospectively investigated post-operative administration of a dendritic cell autologous tumor vaccine in 6 adult progressive glioblastoma patients. Median OS was 36 months. The results here suggest a potential benefit with this vaccine for adjuvant therapy after repeat resection, but as it was only in a small series of patients this was designated Class III evidence [89]. Sakai et al.'s Wilms' Tumor DC vaccine in 6 cases resulted in a mOS of 8.3 months and was deemed safe, but no conclusion was made on efficacy in this Class III designated phase I trial [102]. Yamanaka et al. evaluated a DC vaccine using peripheral blood dendritic cells generated with GMCSF and interleukin-4 (IL4) and pulsed with autologous tumor lysate in 24 patients. They were then vaccinated intratumorally, or both intratumorally and intradermally. Median OS was 15.5 months and the authors determined the vaccination trial proved safety in this Class III designated phase I trial [111].

Another group of vaccination trials utilized autologous tissue and tumors to generate specific, individualized vaccinations. Fakhrai et al. studied a whole-cell vaccine with autologous tumor cells modified with TGFb2 in 6 patients. Median OS was 15.9 months, and of those with an immune response having a median OS of 18.2 months. The comparison cited a historic mOS value of 11 months for conventional treatment, concluding that the humoral and cellular immunity inductions support further evaluation [94]. This was designated Class III as a small, nonrandomized series. Plautz et al. studied systemic adoptive therapy in 10 patients vaccinated with irradiated tumor cells. Median OS was 11.3 months and there was limited data which didn't provide evidence for a clinical benefit [100]. These data were designated Class III. Schijns et al. used Gliovac in 9 patients, found a 40 week OS of $77 \%$, and received approval for development of a phase II clinical trial [104].

Shibao et al. prospectively studied a vaccine targeting angiogenesis factors in 9 progressive glioblastoma patients. There were limited outcome data included, with two patients achieving SD and six PD. The authors reported a preliminary sense of efficacy and safety. As a small series phase I trial, this is class III [106].

Tanaka et al. studied 17 adult patients receiving human umbilical vein endothelial cells. PFS was 5.5 months and authors concluded that although the vaccine had a low radiologic response rate, there was a favorable mOS compared to historical series for patients receiving salvage therapy after a varying number of previous lines of treatment through multiple recurrences [107]. This study warranted Class III designation.

Several peptide vaccination studies were also included. Bloch et al. achieved a PFS of 4.5 months, PFS6 29.3\%, 


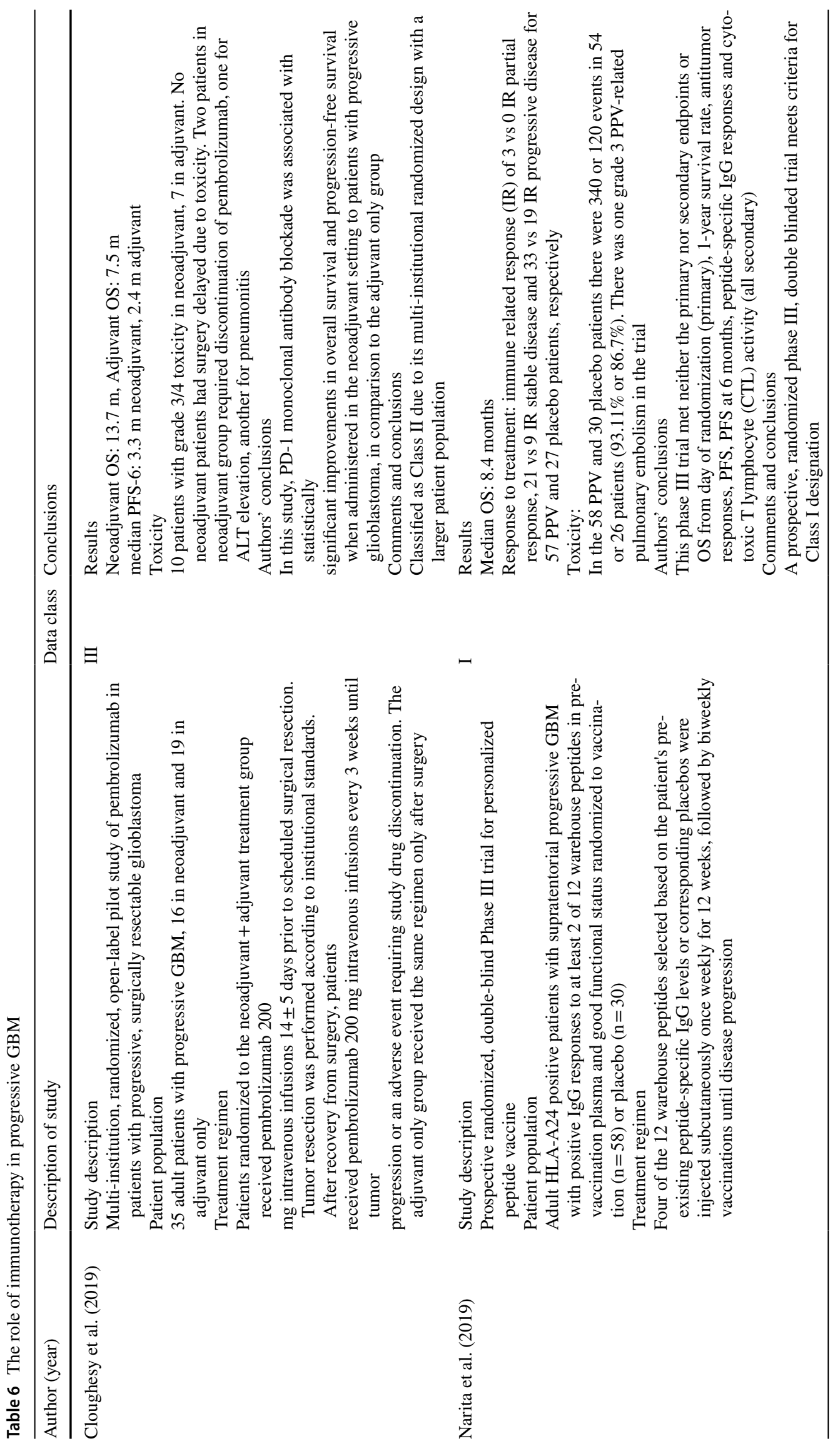




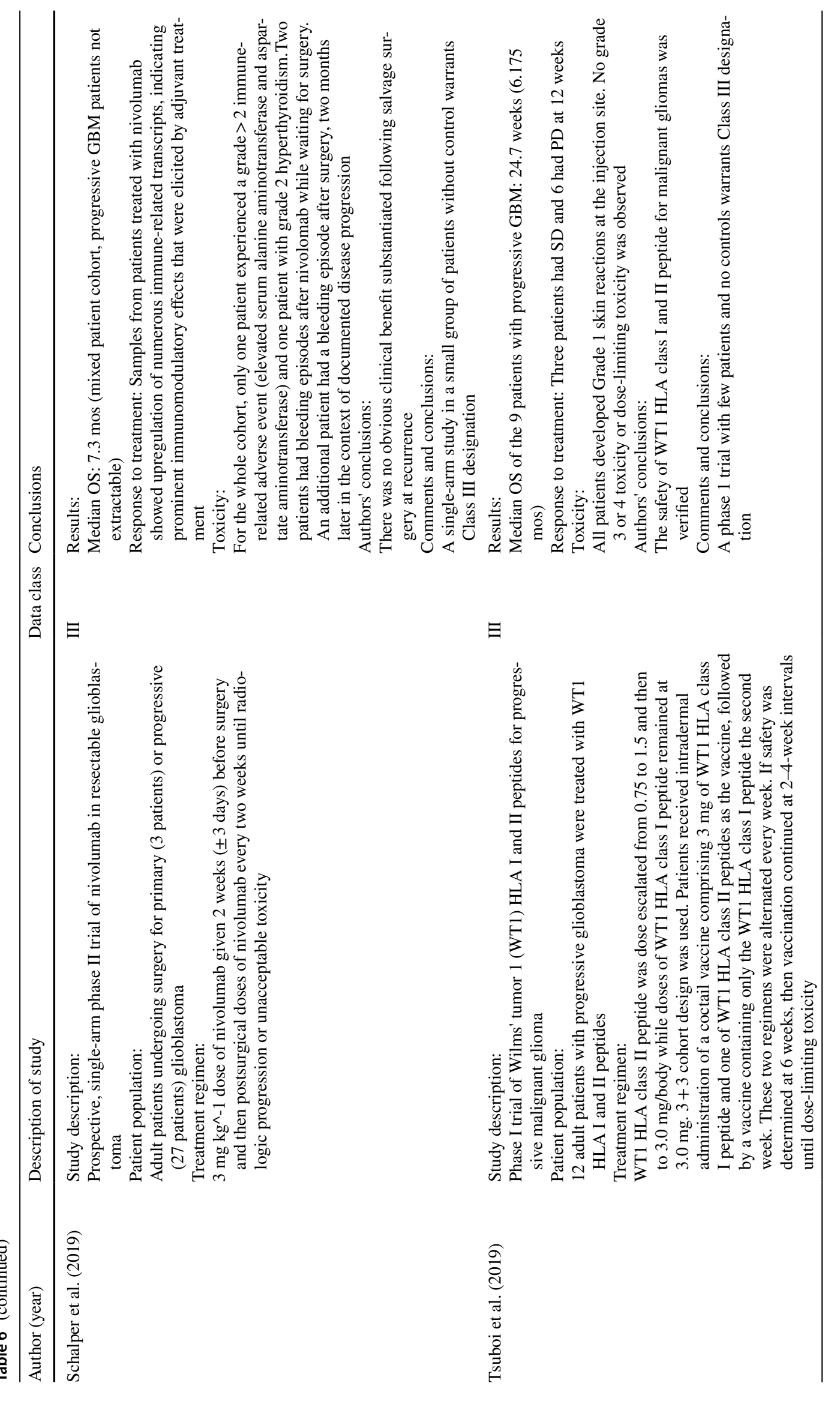




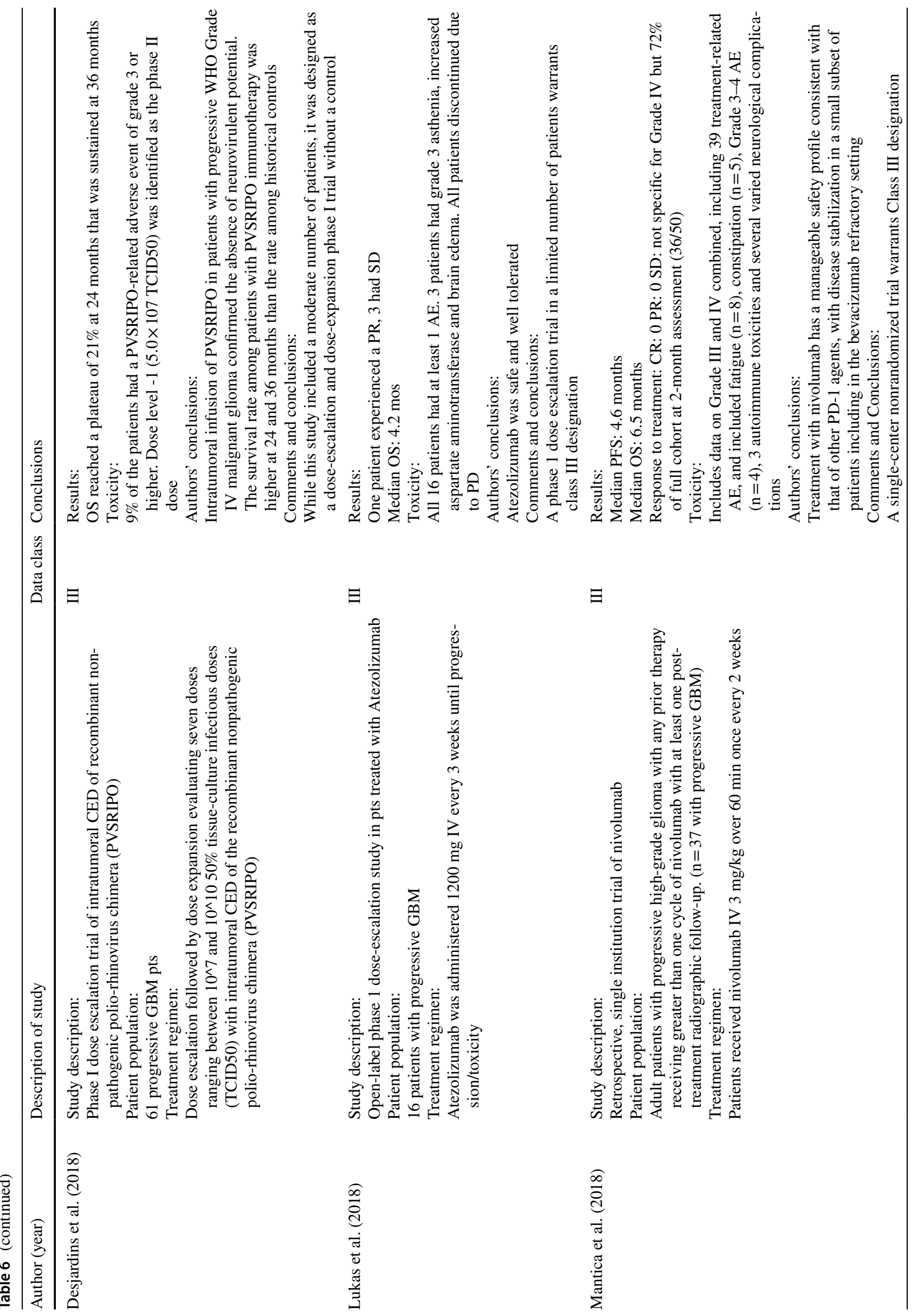




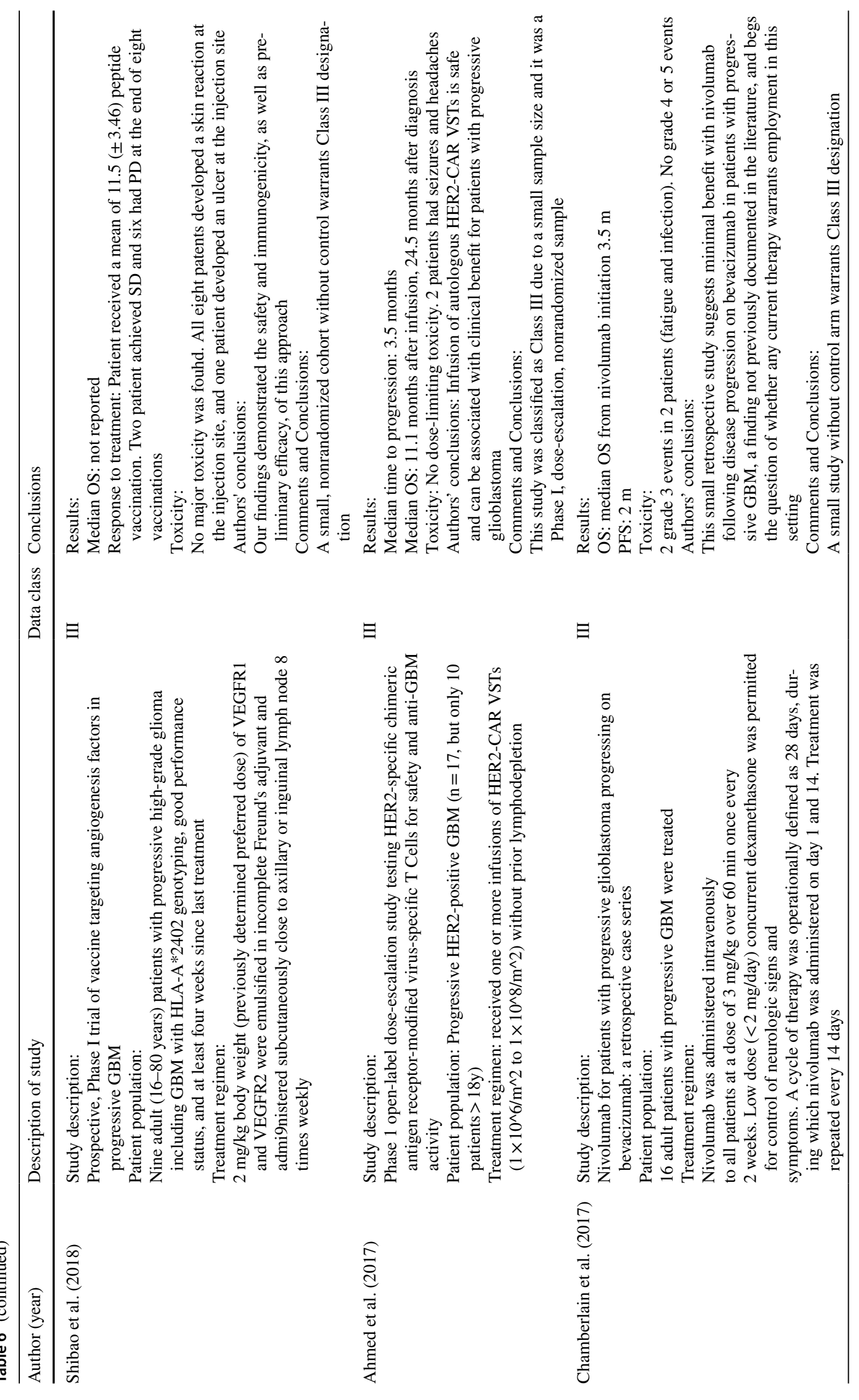




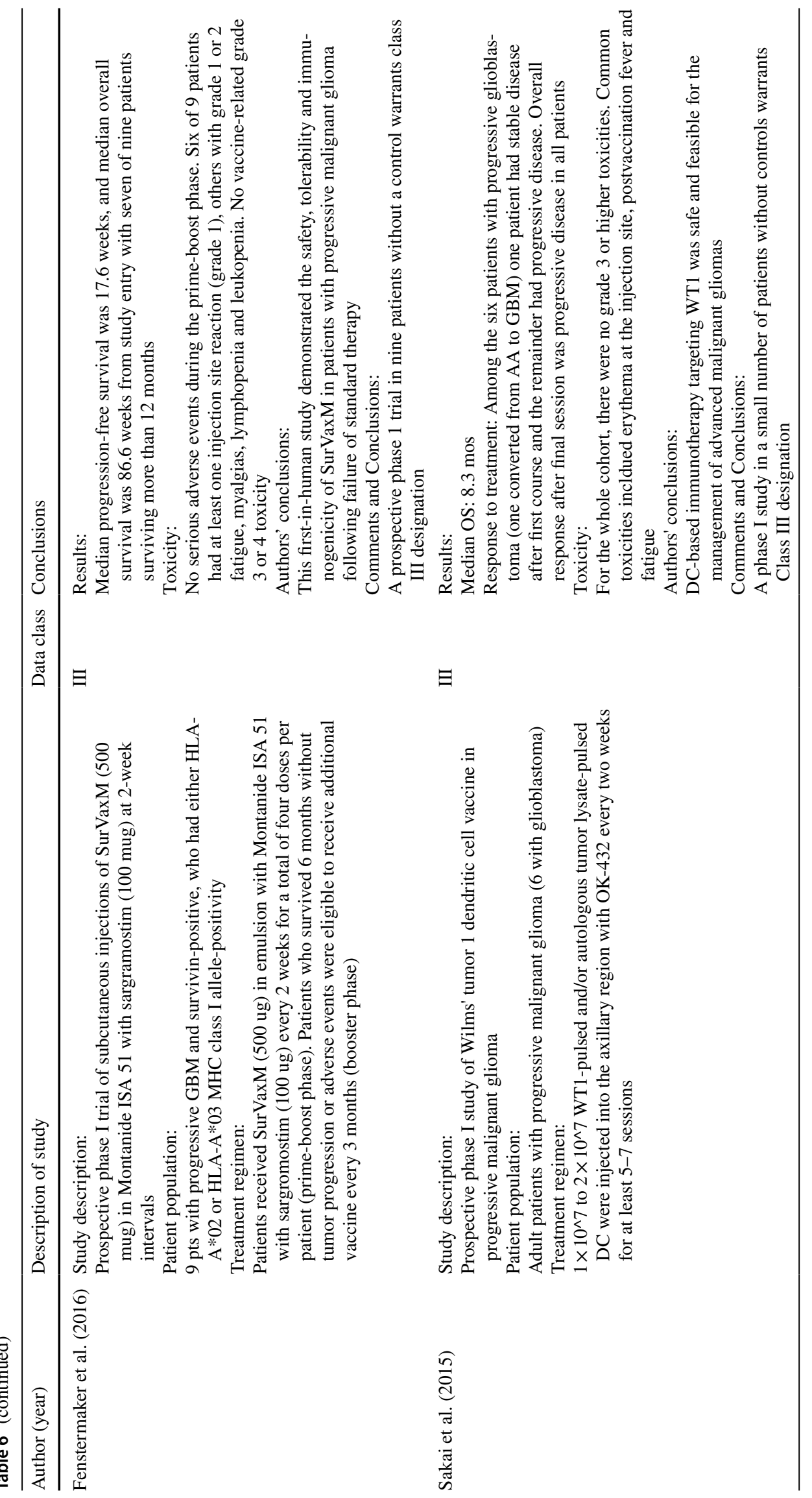




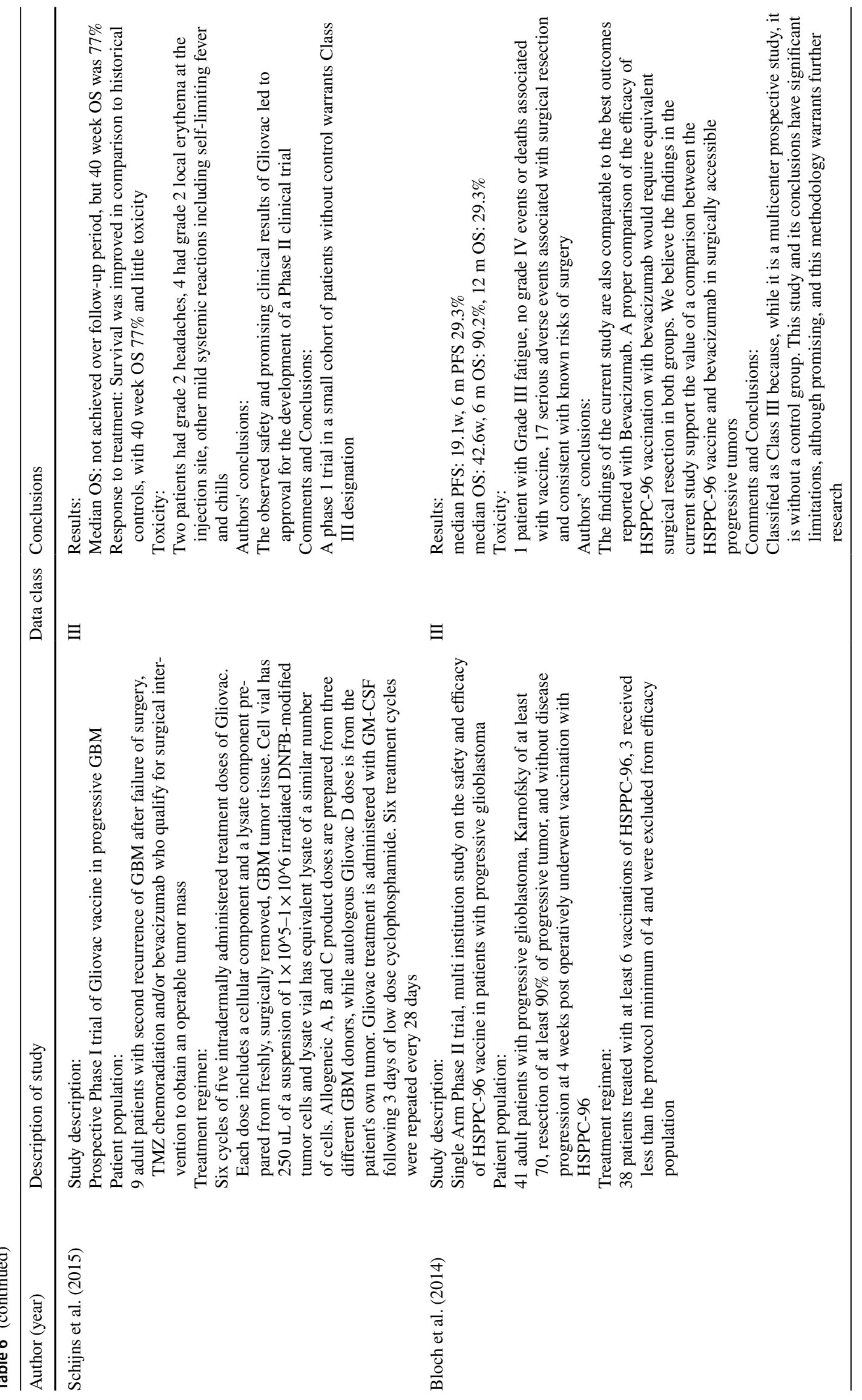




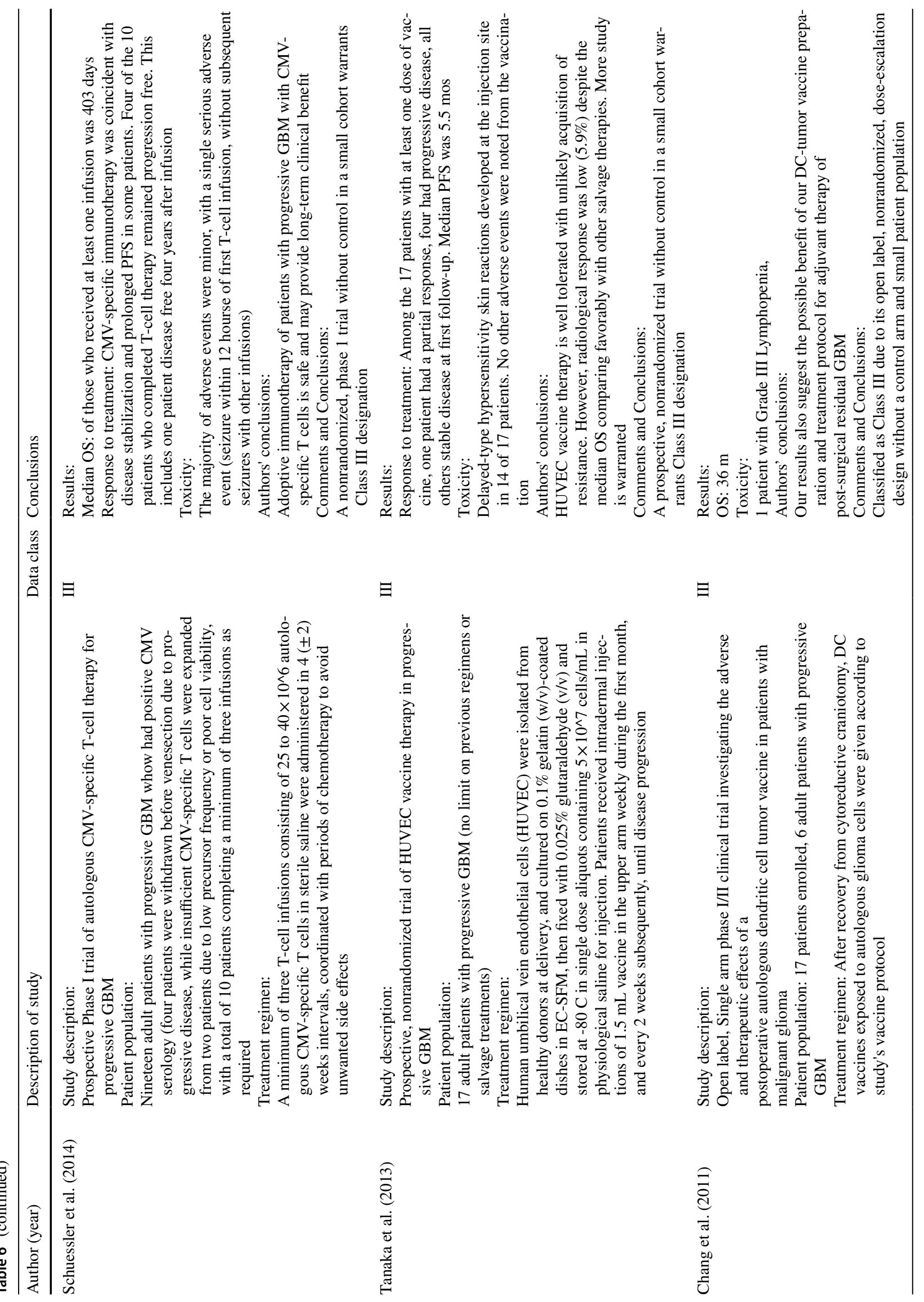




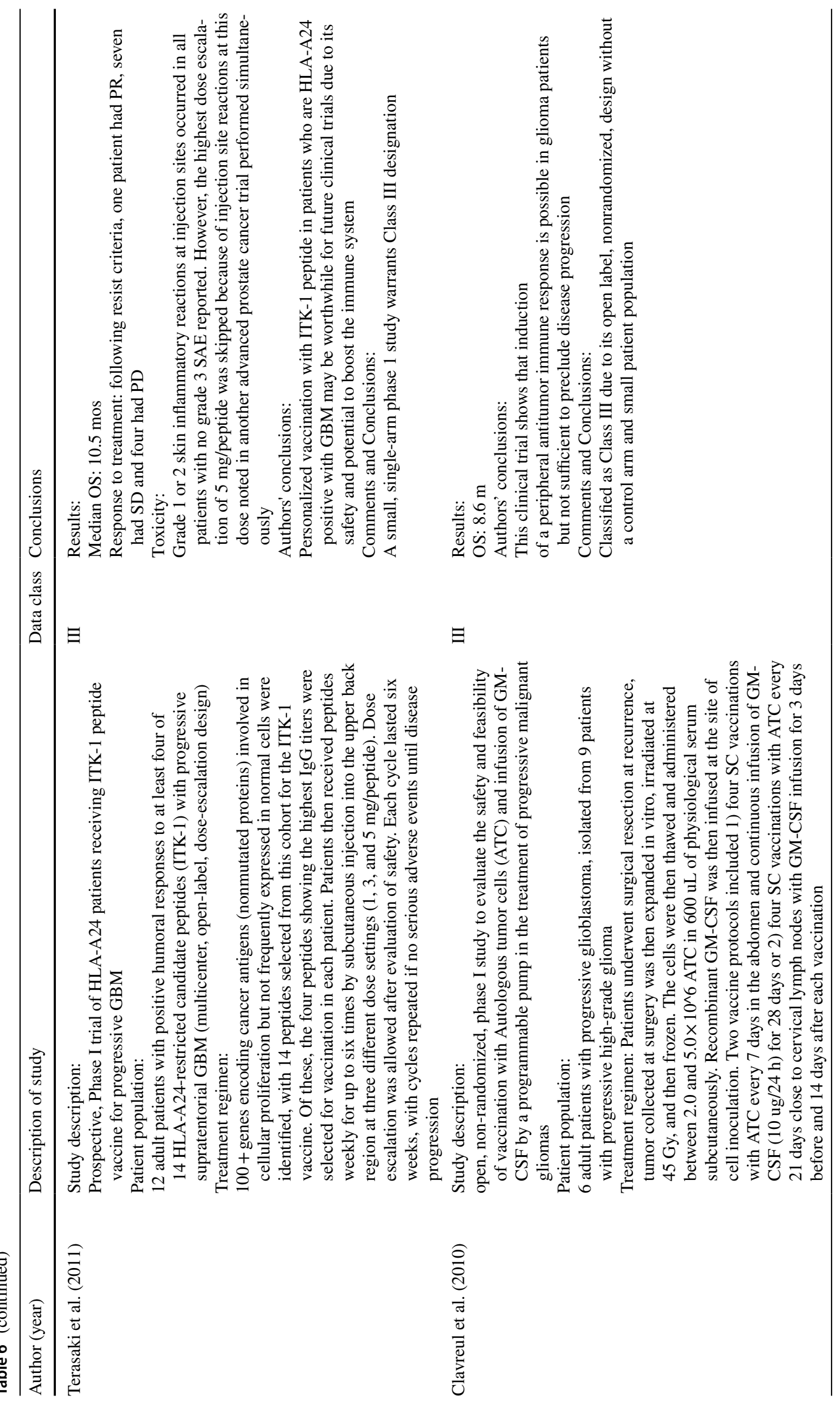




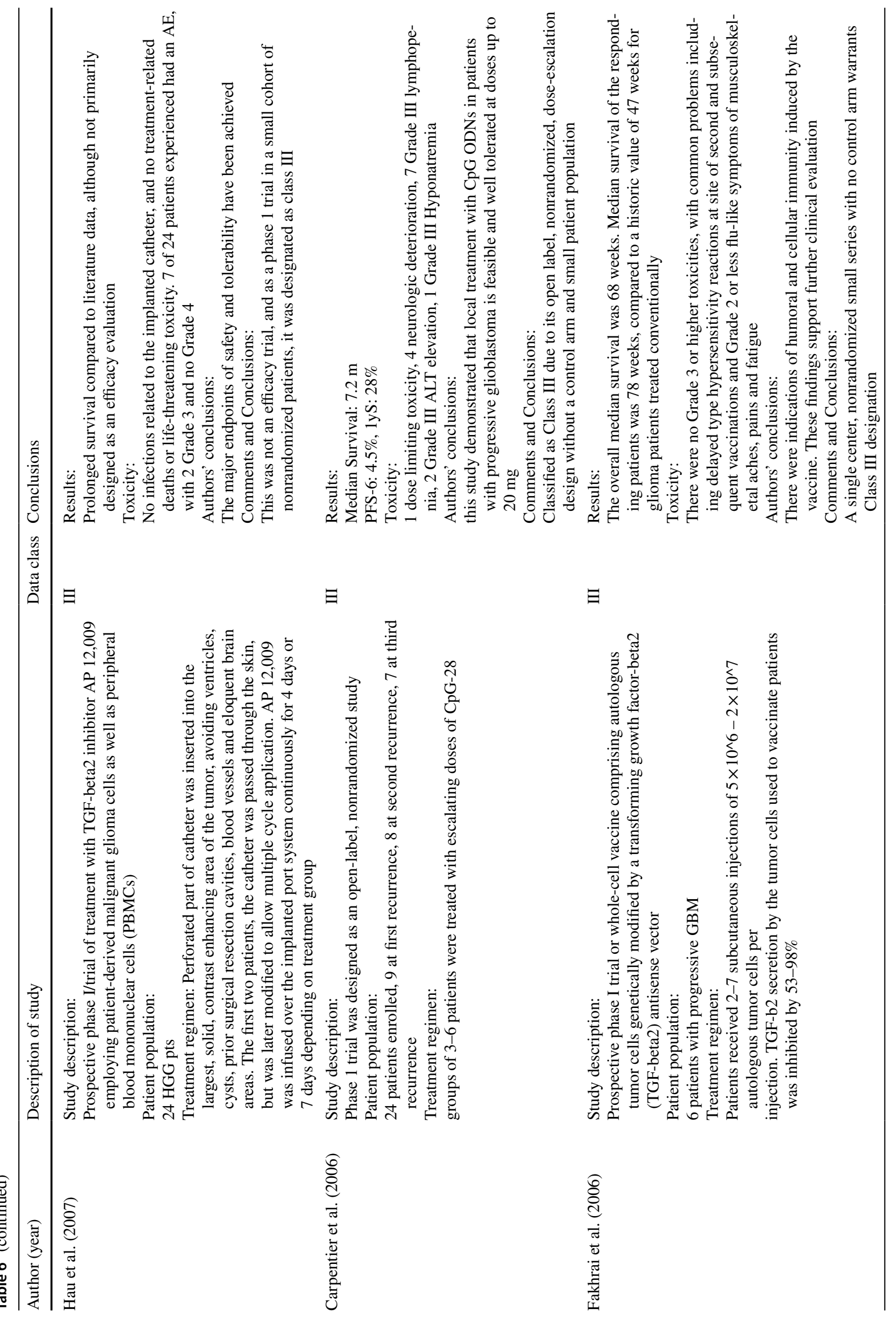




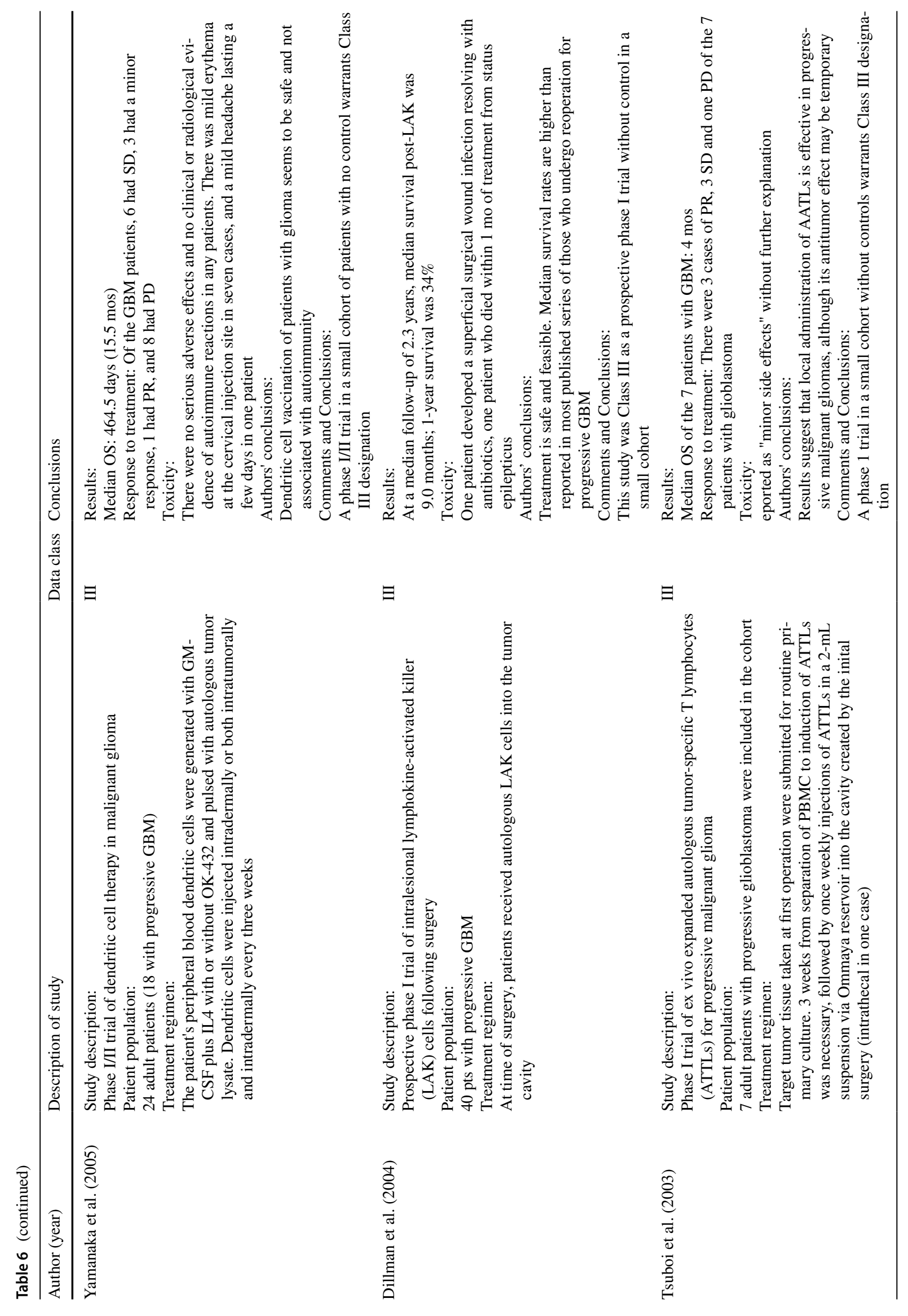




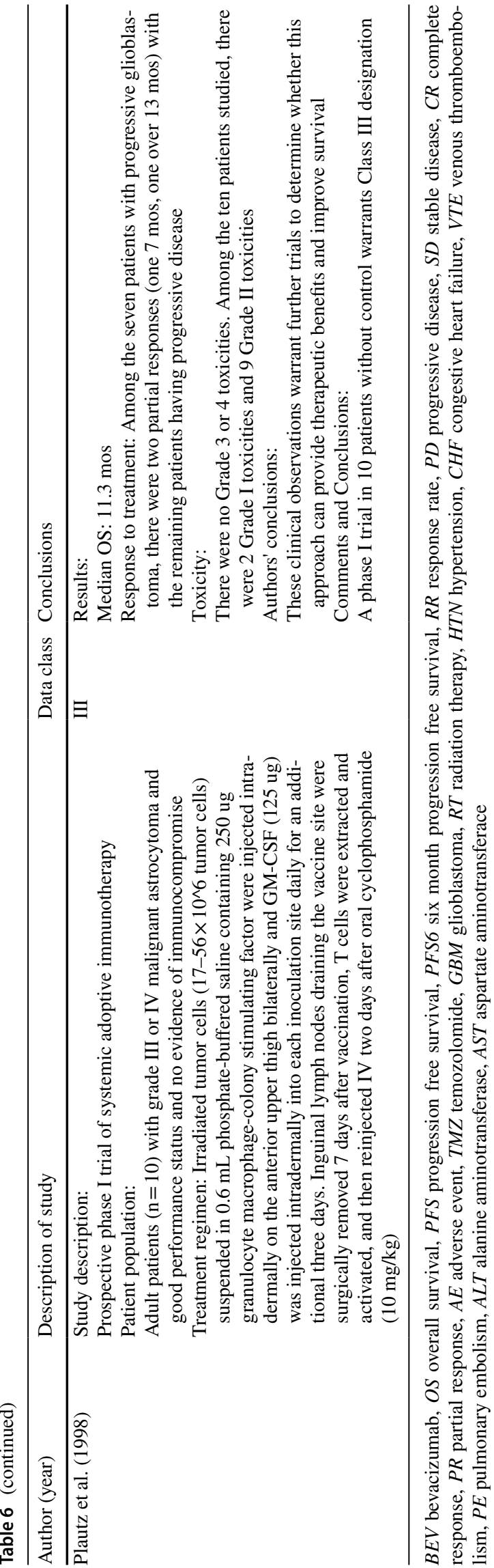

mOS 9.9 months with HSPPC-96. They concluded the findings were comparable to the best outcomes with BEV, however they did not have a direct comparison and data were designated Class III [86]. Fenstermaker et al. performed a phase I trial with SurVaxM in specific HLA subtypes. PFS was 4.1 months and mOS was 20.2 months with 7 patients surviving beyond 12 months, concluding the vaccine was safe and induced an immune response in progressive GBM patients after failure of standard therapy [95]. This was designated Class III for small sample size and a lack of a control group. Teresaki et al. used individualized peptide vaccinations, achieving a mOS 10.5 months with seven patients showing stability of disease. Authors determined the vaccinations may have potential immune system boosting effects and could be worthy of further evaluation [108]. Tsuboi et al. evaluated the Wilms tumor 1 (WT1) peptide vaccination in 9 progressive glioblastoma patients that completed the study. Median OS was 5.8 months and the authors determined this was a safe treatment regimen, but did not comment on efficacy [109]. All peptide vaccination studies were given Class III designation.

In the only Class I designated study in vaccine trials, and imumunotherapy, Narita et al. prospectively evaluated 88 progressive glioblastoma with HLA-A24 positive patients given personalized peptide vaccines. Median OS was 8.4 months and the trial met neither the primary endpoints or secondary endpoints of OS, 1 year survival rate, antitumor responses, PFS, or PFS6, thus deeming the treatment of no significant benefit [99].

\section{Immune checkpoint inhibitors}

Cloughesy et al.'s randomized pilot study of pembrolizumab (inhibitor of PD-1) evaluated 35 patients with surgically resectable progressive glioblastoma. Sixteen patients underwent neoadjujvant and adjuvant treatment while 19 patients received adjuvant therapy only. Median OS in the neoadjuvant group was 13.7 months and 7.5 months in the adjuvant group. Median PFS-6 was 3.3 months in neoadjuvant, 2.4 months in the adjuvant group. The authors concluded PD-1 monoclonal blockade showed a significant OS and PFS benefit in the neoadjuvant group [91]. These data were deemed Class III since, despite being a multi-institutional, randomized design with a large patient population, it was pilot by design. Lukas et al. studied atezolizumab, an antiPDL1 agent, in 16 patients and found the drug to be well tolerated with mOS of 4.2 months, but did not comment on efficacy [97]. This was designated Class III as a doseescalation trial. In another class III study, Reiss et al. found that heavily pretreated patients with high grade gliomas had low response rates to pembrolizumab [101]. 
Three studies utilized nivolumab. Chamberlain et al.'s study of nivolumab, an immune checkpoint inhibitor targeting PD-1, was evaluated retrospectively after progression on BEV. They found mOS from treatment initiation was 3.5 months with PFS 2 months, concluding there was minimal benefit with nivolumab after disease progression on BEV [88]. Schalper et al. also studied nivolumab in resectable glioblastoma as both neoadjuvant and adjuvant therapy, finding no obvious benefit [103]. Mantica et al. reviewed nivolumab and found the treatment was safe and a small subset of patients experienced disease stabilization [98]. All of these studies were considered Class III as two were retrospective and one was a small series without a control.

\section{Other immunotherapy}

Clavreul et al.'s Phase I non-randomized study of Autologous Tumor Cells (ATC) with GM-CSF by subcutaneous programmable pump delivery in 6 patients with progressive glioblastoma was performed after resection at recurrence. Median OS was 8.6 months and the authors concluded there was an immune response induced in patients but not enough to delay or prevent disease progression [90]. This was in a small group of patients without control and designated as Class III evidence.

Dillman et al. prospectively evaluated intralesional lymphokine-activated killer cells (LAKs) in 40 patients after surgery. Median OS was 9 months with a 12-month survival of $34 \%$. The authors concluded that median survival rates were higher than other published series for patients undergoing repeat resection [93]. As it was a relatively small study without a control group, this was deemed Class III.

Hau et al. evaluated AP12009, a TGFb2 inhibitor with patient derived glioma cells and peripheral blood mononuclear cells. In 19 patients undergoing direct tumor delivery, results showed a median OS of 10.3 months, although this was not a primary end point of the safety study. The authors concluded the treatment was safe and well tolerated [96]. This warrants Class III designation.

Carpentier et al.'s study evaluated 24 patients treated at either their first, second, or third recurrence with escalating doses of CpG-28. Median OS was 7.2 months and PFS6 4.5\%- and 12-month survival 28\%. Authors felt the treatment was well tolerated, and as a phase I trial without randomization and a small patient population, this was designated Class III evidence [87].

\section{Convection enhanced delivery}

Desjardins et al. evaluated intratumoral convection enhanced delivery of recombinant polio-rhinovirus chimera in 61 patients as a dose escalation and dose expansion trial. Median OS reached a plateau of $21 \%$ at 24 months which was sustained at 36 months and the authors concluded the treatment was safe and the survival rate was higher than among historical controls and warranted further evaluation [92]. This was deemed Class III as it lacked a control group and was a phase I dose-escalation trial.

\section{Synthesis}

The prior guidelines did not discuss immunotherapeutic regimens for progressive glioblastoma. In this update, we reviewed the studies on convection enhanced delivery, varying T-Cell and autologous lymphocyte or mononuclear cell studies, intratumoral agent delivery, vaccinations, and immune checkpoint inhibitors. The highest-level data were in a class I study showing no obvious benefit of a personalized peptide vaccine and a class III designated study showing a significant survival benefit in the neoadjuvant and adjuvant administration of pembrolizumab, increasing 6.2 months over the adjuvant only treatment group. The remainder of studies were Class III designations and of little benefit in guiding treatment suggestions as they were mostly early, Phase I safety and tolerability trials pending further efficacy study. This precludes the ability to make a recommendation for or against immunotherapy, suggesting the modality still warrants further evaluation. As such it is suggested that when available, and patients meet inclusion criteria, they be enrolled in properly designed studies of immunotherapy.

Question 7 In adults with progressive glioblastoma is the use of immunotherapy in combination with cytotoxic agents superior to standard salvage cytotoxic chemotherapy as measured by progression free survival and overall survival?

Prior Recommendations: In the previously published guidelines on the role of targeted therapies in management of progressive glioblastoma in adults, immunotherapeutic agents in combination with cytotoxic treatments were not reviewed.

\section{Recommendation}

There is insufficient evidence to support a recommendation regarding this question.

\section{Study selection and characteristics of the updated search}

The initial search strategy included 904 candidate articles. A total of 31 articles qualified for full text review. From these, 2 articles were included in the final review for Question 7 and are included in Table $7[112,113]$. 
Table 7 The role of immunotherapy combined with cytotoxic therapy

\begin{tabular}{|c|c|c|c|}
\hline Author (year) & Description of study & Data class & Conclusions \\
\hline Akasaki (2016) & $\begin{array}{l}\text { Study description: } \\
\text { Single institution, Phase I/II trial of safety and } \\
\text { efficacy of TMZ+immunotherapy with fusions of } \\
\text { dendritic cells and glioma cells in progressive and } \\
\text { newly diagnosed glioblastoma } \\
\text { Patient population: } \\
\text { Adult patients with glioblastoma of which } 10 \text { were } \\
\text { progressive and } 22 \text { were newly diagnosed. Review } \\
\text { of the } 10 \text { patients' pathology indicated Glioblas- } \\
\text { toma in } 7 \text {, Anaplastic Astrocytoma for } 2 \text {, and Ana- } \\
\text { plastic Oligodendroglioma in } 1 \\
\text { Treatment regimen: } \\
\text { Group R (progressive, } n=10 \text { ) previously underwent } \\
\text { Surgical resection followed by TMZ Chemother- } \\
\text { apy and Radiation. At recurrence, TMZ was given } \\
\text { at } 150-200 \text { mg/m } 2 / \text { day for } 5 \text { days of each } 28 \text { day } \\
\text { treatment cycle. FCs suspended in } .5 \text { cc normal } \\
\text { saline were inoculated intradermally } 2 \text { weeks after } \\
\text { first maintenance dose of TMZ and repeated at } \\
\text { least } 3 \text { times in each cycle. After } 3 \text { rd inoculation, } \\
\text { FC-inoculation was repeated every } 6 \text { - } 12 \text { months } \\
\text { barring progressive disease }\end{array}$ & III & $\begin{array}{l}\text { Results: } \\
\text { OS: } 18 \mathrm{~m} \text {, stratified for dose of FC inocu- } \\
\text { lation: }<1 \times 10^{\wedge} 612.6 \mathrm{~m}, 1-2 \times 10^{\wedge} 6 \\
\text { 18.2, }>2 \times 10^{\wedge} 616.1 \mathrm{~m} \\
\text { Toxicity: } \\
\text { Injection site Reaction Grade } 1: 4 \text {, Lymphope- } \\
\text { nia/Leukopenia G1: } 5 \text {, Lymphopenia/leuko- } \\
\text { penia G2: } 2 \text {, no adverse events necessitated } \\
\text { halting treatment, no correlation to number of } \\
\text { inocluated FCs } \\
\text { Authors' conclusions: } \\
\text { TMZ+FC immunotherapy may have a capabil- } \\
\text { ity to enhance the TMZ-based standard adju- } \\
\text { vant therapy for patients with GBM and may } \\
\text { have a synergistic effect in progressive GBM } \\
\text { with acquired chemotherapeutic resistance } \\
\text { Comments and conclusions: } \\
\text { A single institution, small nonrandomized } \\
\text { study warrants Class III designation }\end{array}$ \\
\hline Hunn et al. (2015) & $\begin{array}{l}\text { Study description: } \\
\text { Prospective phase I trial of TMZ plus monocyte- } \\
\text { derived dendritic cells (DC) pulsed with autolo- } \\
\text { gous tumor cells } \\
\text { Patient population: } \\
14 \text { pts with progressive GBM, } 9 \text { of whom com- } \\
\text { pleted the trial } \\
\text { Treatment regimen: } \\
\text { Following leukapheresis to harvest PBMC, crani- } \\
\text { otomy for tumor resection occurred. Three weeks } \\
\text { later, patients received a priming course of three } \\
\text { rounds of dendritic cell (DC)-based vaccination } \\
\text { intradermally at } 2 \text {-week intervals, dosing } 4 \times 10^{\wedge} 6 \\
\text { DC. One week after } 3^{\text {rd }} \text { vaccine, patients began } \\
\text { TMZ } 150-200 \mathrm{mg} / \mathrm{m}^{\wedge} 2 \text { orally for } 5 \text { days every } \\
28 \text {-day cycle for up to } 6 \text { cycles. Booster vaccine of } \\
1 \times 10^{\wedge} 6 \text { DC was administered } 2 \text { weeks after each } \\
\text { cycle of TMZ }\end{array}$ & III & $\begin{array}{l}\text { Results: } \\
\text { Nine of } 14 \text { patients completed the initial phase } \\
\text { of priming vaccinations and two cycles of } \\
\text { TMZ, one had radionecrosis, one rapidly } \\
\text { progressed, and in } 3 \text { the yield of DC vaccine } \\
\text { was insufficient to proceed. } 2 \text { had radiological } \\
\text { responses. } 6 \text { mo PFS } 22 \% \\
\text { Toxicity: } \\
\text { There were no grade } 4 \text { toxicities, premature } \\
\text { withdrawals due to treatment-related toxicity, } \\
\text { or AE directly attributable to vaccination } \\
\text { Authors' conclusions: } \\
\text { The combined treatment was safe and well- } \\
\text { tolerated but feasibility in the progressive } \\
\text { setting was marginal. Evidence of immune } \\
\text { responses in a few patients broadly correlated } \\
\text { with better clinical outcome } \\
\text { Comments and conclusions: } \\
\text { A phase } 1 \text { trial without control in a small } \\
\text { cohort warrants Class III designation }\end{array}$ \\
\hline
\end{tabular}

$B E V$ bevacizumab, $O S$ overall survival, $P F S$ progression free survival, $P F S 6$ six month progression free survival, $R R$ response rate, $P D$ progressive disease, $S D$ stable disease, $C R$ complete response, $P R$ partial response, $A E$ adverse event, $T M Z$ temozolomide, $G B M$ glioblastoma, $R T$ radiation therapy

In a Phase I trial of temozolomide with monocyte derived dendritic cells pulsed with autologous tumor cells in 14 patients, only 9 of which were included in study results, PFS6 was $22 \%$. Hunn et al. concluded this strategy was safe and well tolerated, but the feasibility was marginal and of limited benefit [112]. Akasaki et al. performed a Phase I/II trial evaluating TMZ with dendritic cell pulsed with glioma cells in both newly diagnosed and progressive glioblastoma. In the seven progressive glioblastoma cases, results showed a mOS of 18 months, with $1 \times 10^{6} \mathrm{DC}$ dose yielding 12.6 month mOS, the $1-2 \times 10^{6}$ dose an 18.2 month survival, and the $>2 \times 10^{6}$ dose a 16.1 month survival. Ultimately, authors concluded this immunotherapy may work synergistically with $\mathrm{TMZ}$ in progressive glioblastoma patients with acquired TMZ resistance [113]. As these were both small studies with nonrandomized cohorts lacking control groups, these were both designated Class III.

\section{Synthesis}

As an effect of a small number of studies with Class III data and limited patient populations and efficacy results, we 
cannot make a recommendation at this time. It would be difficult to evaluate the effect without further data and larger studies with randomized designs with control groups, otherwise one cannot delineate whether effects are synergistic or related to either the cytotoxic or immunotherapy component of the treatment regimen. Thus, there is insufficient evidence on which to base a recommendation regarding this question. Again, it is suggested that when available, and patients meet inclusion criteria, they be enrolled in properly designed studies of immunotherapy in combination with cytotoxic agents.

Question 8 In adults with progressive glioblastoma is the use of immunotherapy in combination with bevacizumab superior to standard salvage cytotoxic chemotherapy as measured by progression free survival and overall survival?

Prior Recommendations: In the previously published guidelines on the role of targeted therapies in the management of progressive glioblastoma in adults, immunotherapeutic agents combined with bevacizumab were not reviewed.

\section{Recommendation}

There is insufficient evidence to support a recommendation regarding this question.

\section{Study selection and characteristics of the updated search}

The initial search strategy included 904 candidate articles. A total of 31 articles qualified for full text review. From these, 1 article met inclusion criteria for inclusion in the final review for Question 8 and is included in Table 8 [114].

Bota et al. evaluated ER1671 and BEV against BEV monotherapy (placebo) in 9 patients, 5 in the active treatment group and 4 in placebo [114]. ERC1671 consisted of ERC-D (inactivated or irradiated whole tumor cells and lysates from allogeneic and autologous glioblastoma patients in combination with an immune system primer), cyclophosphamide, and GM-CSF. PFS was 7.3 months in the treatment group and 5.4 months in the placebo group. The authors concluded, based on interim results, that there was a meaningful survival benefit with minimal additional toxicity when adding ERC1671, GM-CSF, and cyclophosphamide treatment to BEV. However, the small size of each cohort limits this interpretation and thus this study yields class III data.

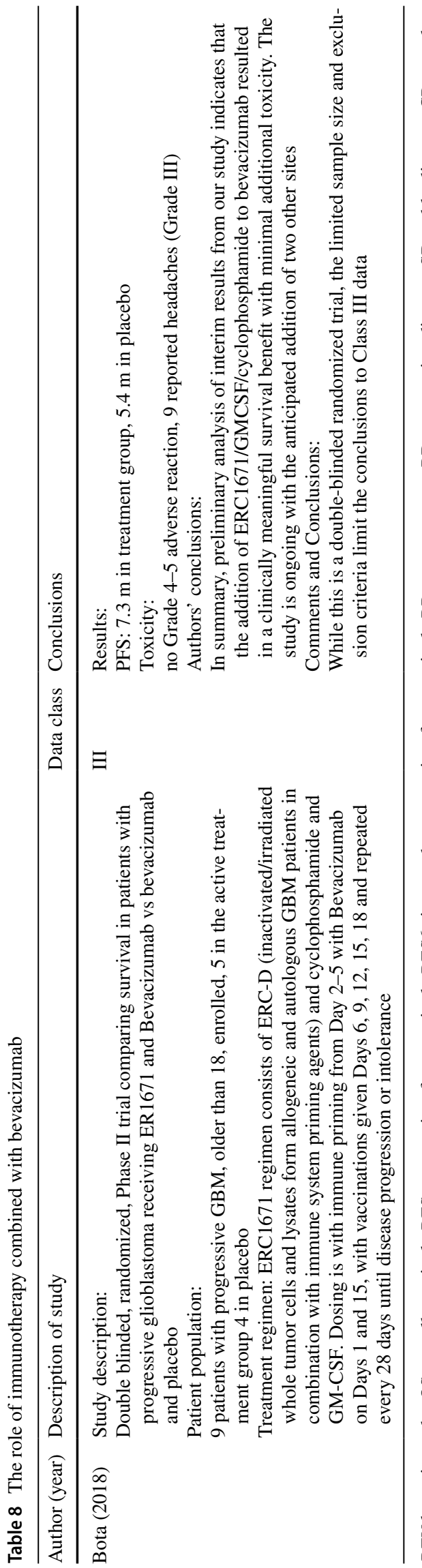




\section{Synthesis}

As there were no combination immunotherapy studies discussed in the prior guidelines, this single study does not provide enough high-level evidence to make a recommendation. Although designated Class III with interim results showing a potentially meaningful benefit over placebo when combining autologous and allogeneic tumor lysates as a vaccination combined with BEV treatment, no recommendations for this question are warranted until larger, randomized studies provide further results. It is suggested that when available, and patients meet inclusion criteria, they be enrolled in properly designed studies of immunotherapy in combination with bevacizumab or other targeted agents.

\section{Discussion}

Treatment for progressive glioblastoma has not significantly changed since our prior guidelines were released, and there has not been a significant change in median overall or progression free survival. Each of our questions were formulated to assess the contribution made by targeted therapies and immunotherapy in the treatment of patients with progressive glioblastoma.

In regard to Question 1, bevacizumab monotherapy was determined to be effective when compared to standard salvage cytotoxic chemotherapy based on improvements in imaging response and progression free survival at six months, although it did not create any significant improvement in median overall survival in progressive glioblastoma patients. Therefore, our recommendation remains unchanged from the prior guidelines released in 2014.

Question 2 sought to address BEV in combination with cytotoxic therapies as a superior treatment regimen to standard salvage cytotoxic therapy. Based on the new available data since 2014, this was found to have no clear survival benefit. More importantly, coupling that lack of evidence with the plethora of information on elevated toxicity in patients receiving BEV in combination with cytotoxic agents, the recommendation was ultimately made Level III against combination therapy. The most important study for this designation with Wick et al., Class I data, with $63.6 \%$ of patients suffering a grade 3 to 5 toxic event.

We then evaluated BEV with other targeted agents in Question 3. Three studies providing data that could answer this question included a phase I dose escalation trial and two small nonrandomized studies. There wasn't evidence of high enough power or quality to develop a recommendation, thus further studies need to be performed to further elucidate the potential of this type of regimen.

Question 4 revolved around targeted therapies in relation to standard salvage cytotoxic treatment in progressive glioblastoma. The highest class data evaluated was Class II, including Brown et al. in their evaluation of cediranib with gorafenib with only a trend toward improved survival, and Wick et al. discussing CD95 pathway inhibition with reirradiation. The remainder of studies did not provide enough evidence of quality to provide a recommendation for or against targeted therapies alone. As such, no recommendation could be made aside from requesting further studies be performed.

When combining targeted with cytotoxic therapy, we also were unable to provide a recommendation. The highest class evidence was Class I, where Batchelor et al. studied cediranib and did not find PFS prolongation in monotherapy or in combination with lomustine [80]. Two class II studies also did not find any survival benefit [78, 79].

Immunotherapeutics were a new addition to this set of recommendations. Ultimately, all groups studied under the question of immunotherapy as monotherapy, in combination with targeted agents, or in combination with BEV (questions 5-8) did not reveal sufficient data to provide any recommendations. The highest class designations were a class I study where Narita et al.'s Phase III trial of a personalized peptide vaccine found no benefit, and a class II study by Cloughesy et al. where they discovered a benefit in mOS with neoadjuvant administration of pembrolizumab [91, 99]. Immune checkpoint inhibitors remain of potential interest, albeit present data does not allow us to provide a recommendation.

\section{Key issues for future research}

Targeted and immunotherapies have generated a significant amount of interest and early research into the treatment of recurrent glioblastoma. Unfortunately, there remains insufficient evidence for the recommended use of many of these agents. However, there were some intriguing findings from this review, specifically the potential benefit of treatment with immune checkpoint inhibition prior to and after surgical resection for progressive glioblastoma. This should generate further studies surrounding immunotherapeutics either as monotherapy, in conjunction with resection, or as combination therapies. Additional well designed, larger, randomized controlled clinical trials may provide additional information on this topic.

\section{Conclusion}

This set of recommendations does not substantially affect the current targeted and immunotherapy regimens being offered to patients with progressive glioblastoma. Ideally, clinical trials should include more patients to better evaluate the safety and survival benefits provided. We did make a 
Level III recommendation against bevacizumab in combination with cytotoxic agents due to the elevated toxicity and lack of benefit in survival, as well as a Level III supporting the use of bevacizumab monotherapy. However, this is not a significant deviation from the previously published guideline on this topic.

To restate, this should not ultimately change the treatment strategies offered by neuro-oncologic specialists in terms of standard treatment regimens. However, there were some intriguing findings, specifically the potential benefit of treatment with immune checkpoint inhibition prior to and after surgical resection for progressive glioblastoma. This should generate further interesting studies surrounding immunotherapeutics either as monotherapy, in conjunction with resection, or as combination therapies.

Ultimately, several limitations exist within our analysis of the literature as well as within the general study designs that prevent our ability to make high-level recommendations. Our search was limited to completed trials with accessible, published data. Our exclusion criteria were strict, resulting in some trials with potentially positive findings and data being excluded due to survival data mixing in pediatric patients and/or WHO Grade III astrocytomas or gliosarcoma. However, this allowed us to restrict our analysis to pay very strict attention to and make recommendations for treatment of adult patients with progressive glioblastoma. On the other hand, we were limited in making strong recommendations as many of the trials were statistically underpowered, lacked control groups, sometimes lacked standardization, or have not yet proceeded beyond initial Phase I and Phase II evaluations. In the future, it may be possible that recommendations on these topics will be strengthened due to creation of larger, randomized, multicenter trials addressing the topics of the smaller studies used as evidence in this document.

Acknowledgements The guidelines task force would like to acknowledge the Congress of Neurological Surgeons Guidelines Committee for their contributions throughout the development of the guideline, and the American Association of Neurological Surgeons/Congress of Neurological Surgeons Joint Guidelines Review Committee for their review, comments, and suggestions throughout peer review, as well as the contributions of Trish Rehring, MPH, CHES, Senior Manager of Clinical Practice Guidelines for the CNS, and Mary Bodach, MLIS, from the Congress of Neurological Surgeons Guidelines Office for organizational assistance and reference librarian services, respectively as well as Jeremy Kupsco, PhD, Informationist, Emory University, for their valuable input as Medical Research Librarians. Throughout the review process, the reviewers and authors were blinded from one another. At this time the guidelines task force would like to acknowledge the following individual peer reviewers for their contributions: John O'Toole, MD, Brian Howard, MD, Jamie Van Gompel, MD, Howard Silberstein, MD, Navid Redjal, MD and Shawn HerveyJumper, MD.

Disclaimer of liability This clinical systematic review and evidencebased guideline was developed by a multidisciplinary physician volunteer task force and serves as an educational tool designed to provide an accurate review of the subject matter covered. These guidelines are disseminated with the understanding that the recommendations by the authors and consultants who have collaborated in their development are not meant to replace the individualized care and treatment advice from a patient's physician(s). If medical advice or assistance is required, the services of a competent physician should be sought. The proposals contained in these guidelines may not be suitable for use in all circumstances. The choice to implement any particular recommendation contained in these guidelines must be made by a managing physician in light of the situation in each particular patient and on the basis of existing resources.

Author contributions The author listed on this publication agrees with the content included and gives explicit consent to the submission of this publication. The author obtained consent from the responsible authorities at the institute/organization where the work has been carried out, before the work was submitted. The author whose name appear on this submission: (1) made substantial contributions to the conception or design of the work; or the acquisition, analysis, or interpretation of data; or the creation of new software used in the work; (2) drafted the work or revised it critically for important intellectual content; (3) approved the version to be published; and (4) agreed to be accountable for all aspects of the work in ensuring that questions related to the accuracy or integrity of any part of the work are appropriately investigated and resolved.

Funding These guidelines were funded exclusively by the Congress of Neurological Surgery and the Joint Section on Tumors of the Congress of Neurological Surgeons and the American Association of Neurological Surgeons, which received no funding from any outside commercial sources to support the development of this document.

Data availability The data generated during and/or analyzed during the current study are available via www.cns.org/guidelines.

\section{Declarations}

Conflict of interest All Guideline Task Force members were required to disclose all potential COIs prior to beginning work on the guideline, using the COI disclosure form of the AANS/CNS Joint Guidelines Review Committee. The CNS Guidelines Committee and Guideline Task Force Chair reviewed the disclosures and either approved or disapproved the nomination and participation on the task force. The CNS Guidelines Committee and Guideline Task Force Chair may approve nominations of task force members with possible conflicts and restrict the writing, reviewing, and/or voting privileges of that person to topics that are unrelated to the possible COIs. The authors have no personal, financial, or institutional interest in any of the drugs, materials, or devices described in this series of articles.

Ethical approval This article does not contain any studies with human participants performed by any of the authors.

Data transparency The author has ensured all data and materials as well as software applications or custom code supports their published claims and comply with field standards.

Disclosures These guidelines were funded exclusively by the Congress of Neurological Surgery and the Joint Section on Tumors of the Congress of Neurological Surgeons and the American Association of Neurological Surgeons, which received no funding from any outside commercial sources to support the development of this document. 


\section{References}

1. Ryken TC, Aygun N, Morris J et al (2014) The role of imaging in the management of progressive glioblastoma: a systematic review and evidence-based clinical practice guideline. J Neurooncol 118:435-460

2. Stupp R, Mason WP, van den Bent MJ et al (2005) Radiotherapy plus concomitant and adjuvant temozolomide for glioblastoma. N Engl J Med 352:987-996

3. Yong RL, Wu T, Mihatov N et al (2014) Residual tumor volume and patient survival following reoperation for recurrent glioblastoma. J Neurosurg 121:802-809

4. Wu W, Lamborn KR, Buckner JC et al (2010) Joint NCCTG and NABTC prognostic factors analysis for high-grade recurrent glioma. Neuro Oncol 12:164-172

5. De Bonis P, Fiorentino A, Anile C et al (2013) The impact of repeated surgery and adjuvant therapy on survival for patients with recurrent glioblastoma. Clin Neurol Neurosurg 115:883-886

6. Zhang L, Ajiferuke I, Sampson M (2006) Optimizing search strategies to identify randomized controlled trials in MEDLINE. BMC Med Res Methodol 6:23

7. Wong SS, Wilczynski NL, Haynes RB (2006) Comparison of top-performing search strategies for detecting clinically sound treatment studies and systematic reviews in MEDLINE and EMBASE. J Med Libr Assoc 94:451-455

8. Wilczynski NL, Haynes RB, Hedges T (2007) EMBASE search strategies achieved high sensitivity and specificity for retrieving methodologically sound systematic reviews. J Clin Epidemiol 60:29-33

9. Wilczynski NL, Haynes RB, Hedges T (2004) Developing optimal search strategies for detecting clinically sound prognostic studies in MEDLINE: an analytic survey. BMC Med 2:23

10. Topfer LA, Parada A, Menon D, Noorani H, Perras C, SerraPrat M (1999) Comparison of literature searches on quality and costs for health technology assessment using the MEDLINE and EMBASE databases. Int $\mathbf{J}$ Technol Assess Health Care 15:297-303

11. Montori VM, Wilczynski NL, Morgan D, Haynes RB, Hedges $\mathrm{T}$ (2005) Optimal search strategies for retrieving systematic reviews from Medline: analytical survey. BMJ 330:68

12. Kastner M, Wilczynski NL, Walker-Dilks C, McKibbon KA, Haynes B (2006) Age-specific search strategies for Medline. J Med Internet Res 8:e25

13. Haynes RB, McKibbon KA, Wilczynski NL, Walter SD, Werre SR, Hedges T (2005) Optimal search strategies for retrieving scientifically strong studies of treatment from Medline: analytical survey. BMJ 330:1179

14. Cai LB, Li J, Lai MY et al (2013) Bevacizumab rescue therapy extends the survival in patients with recurrent malignant glioma. Chin J Cancer Res 25:206-211

15. Flieger M, Ganswindt U, Schwarz SB et al (2014) Re-irradiation and bevacizumab in recurrent high-grade glioma: an effective treatment option. J Neurooncol 117:337-345

16. Kaloshi G, Brace G, Rroji A et al (2013) Bevacizumab alone at $5 \mathrm{mg} / \mathrm{kg}$ in an every-3-week schedule for patients with recurrent glioblastomas: a single center experience. Tumori 99:601-603

17. Nagane M, Nishikawa R, Narita Y et al (2012) Phase II study of single-agent bevacizumab in Japanese patients with recurrent malignant glioma. Jpn J Clin Oncol 42:887-895

18. Piccioni DE, Selfridge J, Mody RR et al (2014) Deferred use of bevacizumab for recurrent glioblastoma is not associated with diminished efficacy. Neuro Oncol 16:815-822

19. Wenger KJ, Wagner M, You SJ et al (2017) Bevacizumab as a last-line treatment for glioblastoma following failure of radiotherapy, temozolomide and lomustine. Oncol Lett 14:1141-1146

20. Balana C, Estival A, Pineda E et al (2017) Prolonged survival after bevacizumab rechallenge in glioblastoma patients with previous response to bevacizumab(dagger). Neurooncol Pract 4:15-23

21. Raizer JJ, Grimm S, Chamberlain MC et al (2010) A phase 2 trial of single-agent bevacizumab given in an every-3-week schedule for patients with recurrent high-grade gliomas. Cancer 116:5297-5305

22. Kreisl TN, Kim L, Moore K et al (2009) Phase II trial of singleagent bevacizumab followed by bevacizumab plus irinotecan at tumor progression in recurrent glioblastoma. J Clin Oncol 27:740-745

23. Friedman HS, Prados MD, Wen PY et al (2009) Bevacizumab alone and in combination with irinotecan in recurrent glioblastoma. J Clin Oncol 27:4733-4740

24. Vredenburgh JJ, Desjardins A, Herndon JE 2nd et al (2007) Phase II trial of bevacizumab and irinotecan in recurrent malignant glioma. Clin Cancer Res 13:1253-1259

25. Arakawa Y, Mizowaki T, Murata D et al (2013) Retrospective analysis of bevacizumab in combination with ifosfamide, carboplatin, and etoposide in patients with second recurrence of glioblastoma. Neurol Med Chir (Tokyo) 53:779-785

26. Badruddoja MA, Pazzi M, Sanan A et al (2017) Phase II study of bi-weekly temozolomide plus bevacizumab for adult patients with recurrent glioblastoma. Cancer Chemother Pharmacol 80:715-721

27. Brandes AA, Finocchiaro G, Zagonel V et al (2016) AVAREG: a phase II, randomized, noncomparative study of fotemustine or bevacizumab for patients with recurrent glioblastoma. Neuro Oncol 18:1304-1312

28. Brandes AA, Gil-Gil M, Saran F et al (2019) A randomized Phase II Trial (TAMIGA) evaluating the efficacy and safety of continuous bevacizumab through multiple lines of treatment for recurrent glioblastoma. Oncologist 24:521-528

29. Brenner A, Zuniga R, Sun JD et al (2018) Hypoxia-activated evofosfamide for treatment of recurrent bevacizumab-refractory glioblastoma: a phase I surgical study. Neuro Oncol 20:1231-1239

30. Field KM, Simes J, Nowak AK et al (2015) Randomized phase 2 study of carboplatin and bevacizumab in recurrent glioblastoma. Neuro Oncol 17:1504-1513

31. Gil MJ, de Las PR, Reynes G et al (2012) Bevacizumab plus irinotecan in recurrent malignant glioma shows high overall survival in a multicenter retrospective pooled series of the Spanish Neuro-Oncology Research Group (GEINO). Anticancer Drugs 23:659-665

32. Gilbert MR, Pugh SL, Aldape K et al (2017) NRG oncology RTOG 0625: a randomized phase II trial of bevacizumab with either irinotecan or dose-dense temozolomide in recurrent glioblastoma. J Neurooncol 131:193-199

33. Heiland DH, Masalha W, Franco P, Machein MR, Weyerbrock A (2016) Progression-free and overall survival in patients with recurrent Glioblastoma multiforme treated with last-line bevacizumab versus bevacizumab/lomustine. J Neurooncol 126:567-575

34. Jakobsen JN, Urup T, Grunnet K et al (2018) Toxicity and efficacy of lomustine and bevacizumab in recurrent glioblastoma patients. J Neurooncol 137:439-446

35. Johansen MD, Urup T, Holst CB et al (2018) Outcome of bevacizumab therapy in patients with recurrent glioblastoma treated with angiotensin system inhibitors. Cancer Invest 36:512-519

36. Pasqualetti F, Pace A, Gonnelli A et al (2018) Single-agent bevacizumab in recurrent glioblastoma after second-line chemotherapy with fotemustine: the experience of the Italian association of neuro-oncology. Am J Clin Oncol 41:1272-1275 
37. Peters KB, Lipp ES, Miller E et al (2018) Phase I/II trial of vorinostat, bevacizumab, and daily temozolomide for recurrent malignant gliomas. J Neurooncol 137:349-356

38. Rahman R, Hempfling K, Norden AD et al (2014) Retrospective study of carmustine or lomustine with bevacizumab in recurrent glioblastoma patients who have failed prior bevacizumab. Neuro Oncol 16:1523-1529

39. Sepulveda JM, Belda-Iniesta C, Gil-Gil M et al (2015) A phase II study of feasibility and toxicity of bevacizumab in combination with temozolomide in patients with recurrent glioblastoma. Clin Transl Oncol 17:743-750

40. Taal W, Oosterkamp HM, Walenkamp AM et al (2014) Singleagent bevacizumab or lomustine versus a combination of bevacizumab plus lomustine in patients with recurrent glioblastoma (BELOB trial): a randomised controlled phase 2 trial. Lancet Oncol 15:943-953

41. Vaccaro V, Fabi A, Vidiri A et al (2014) Activity and safety of bevacizumab plus fotemustine for recurrent malignant gliomas. Biomed Res Int 2014:351252

42. Weathers SP, Han X, Liu DD et al (2016) A randomized phase II trial of standard dose bevacizumab versus low dose bevacizumab plus lomustine (CCNU) in adults with recurrent glioblastoma. J Neurooncol 129:487-494

43. Wick W, Gorlia T, Bendszus M et al (2017) Lomustine and bevacizumab in progressive glioblastoma. N Engl J Med 377:1954-1963

44. Mrugala MM, Crew LK, Fink JR, Spence AM (2012) Carboplatin and bevacizumab for recurrent malignant glioma. Oncol Lett 4:1082-1086

45. Odia Y, Iwamoto FM, Moustakas A et al (2016) A phase II trial of enzastaurin (LY317615) in combination with bevacizumab in adults with recurrent malignant gliomas. J Neurooncol 127:127-135

46. Lassen U, Chinot OL, McBain C et al (2015) Phase 1 doseescalation study of the antiplacental growth factor monoclonal antibody RO5323441 combined with bevacizumab in patients with recurrent glioblastoma. Neuro Oncol 17:1007-1015

47. D'Alessandris QG, Montano N, Cenci T et al (2013) Targeted therapy with bevacizumab and erlotinib tailored to the molecular profile of patients with recurrent glioblastoma. Preliminary experience. Acta Neurochir (Wien) 155:33-40

48. Odia Y, Sul J, Shih JH et al (2016) A Phase II trial of tandutinib (MLN 518) in combination with bevacizumab for patients with recurrent glioblastoma. CNS Oncol 5:59-67

49. Wuthrick EJ, Curran WJ Jr, Camphausen K et al (2014) A pilot study of hypofractionated stereotactic radiation therapy and sunitinib in previously irradiated patients with recurrent high-grade glioma. Int J Radiat Oncol Biol Phys 90:369-375

50. Wick W, Fricke H, Junge K et al (2014) A phase II, randomized, study of weekly APG101+reirradiation versus reirradiation in progressive glioblastoma. Clin Cancer Res 20:6304-6313

51. Wen PY, Touat M, Alexander BM et al (2019) Buparlisib in patients with recurrent glioblastoma harboring phosphatidylinositol 3-kinase pathway activation: an open-label, multicenter, multi-arm, phase II trial. J Clin Oncol 37:741-750

52. Pitz MW, Eisenhauer EA, MacNeil MV et al (2015) Phase II study of PX-866 in recurrent glioblastoma. Neuro Oncol 17:1270-1274

53. Phuphanich S, Raizer J, Chamberlain M et al (2017) Phase II study of MEDI-575, an anti-platelet-derived growth factor-alpha antibody, in patients with recurrent glioblastoma. J Neurooncol 131:185-191

54. Pan E, Yu D, Yue B et al (2012) A prospective phase II singleinstitution trial of sunitinib for recurrent malignant glioma. $\mathrm{J}$ Neurooncol 110:111-118
55. Muhic A, Poulsen HS, Sorensen M, Grunnet K, Lassen U (2013) Phase II open-label study of nintedanib in patients with recurrent glioblastoma multiforme. J Neurooncol 111:205-212

56. Lombardi G, De Salvo GL, Brandes AA et al (2019) Regorafenib compared with lomustine in patients with relapsed glioblastoma (REGOMA): a multicentre, open-label, randomised, controlled, phase 2 trial. Lancet Oncol 20:110-119

57. Lassman AB, van den Bent MJ, Gan HK et al (2019) Safety and efficacy of depatuxizumab mafodotin + temozolomide in patients with EGFR-amplified, recurrent glioblastoma: results from an international phase I multicenter trial. Neuro Oncol 21:106-114

58. Lassman AB, Pugh SL, Gilbert MR et al (2015) Phase 2 trial of dasatinib in target-selected patients with recurrent glioblastoma (RTOG 0627). Neuro Oncol 17:992-998

59. Krolicki L, Bruchertseifer F, Kunikowska J et al (2019) Safety and efficacy of targeted alpha therapy with (213)Bi-DOTA-substance $\mathrm{P}$ in recurrent glioblastoma. Eur J Nucl Med Mol Imaging 46:614-622

60. Kalpathy-Cramer J, Chandra V, Da X et al (2017) Phase II study of tivozanib, an oral VEGFR inhibitor, in patients with recurrent glioblastoma. J Neurooncol 131:603-610

61. Jia PF, Gu WT, Zhang WF, Li F (2015) Treatment of recurrent malignant gliomas with 13-cis-retinoic acid naphthalene triazole. Neurol Sci 36:717-721

62. Hassler MR, Vedadinejad M, Flechl B et al (2014) Response to imatinib as a function of target kinase expression in recurrent glioblastoma. Springerplus 3:111

63. Gerstner ER, Ye X, Duda DG et al (2015) A phase I study of cediranib in combination with cilengitide in patients with recurrent glioblastoma. Neuro Oncol 17:1386-1392

64. Gan HK, Reardon DA, Lassman AB et al (2018) Safety, pharmacokinetics, and antitumor response of depatuxizumab mafodotin as monotherapy or in combination with temozolomide in patients with glioblastoma. Neuro Oncol 20:838-847

65. Gallego O, Cuatrecasas M, Benavides M et al (2014) Efficacy of erlotinib in patients with relapsed gliobastoma multiforme who expressed EGFRVIII and PTEN determined by immunohistochemistry. J Neurooncol 116:413-419

66. Butowski N, Colman H, De Groot JF et al (2016) Orally administered colony stimulating factor 1 receptor inhibitor PLX3397 in recurrent glioblastoma: an Ivy foundation early phase clinical trials consortium phase II study. Neuro Oncol 18:557-564

67. Brown N, McBain C, Nash S et al (2016) Multi-center randomized phase II study comparing cediranib plus gefitinib with cediranib plus placebo in subjects with recurrent/progressive glioblastoma. PLoS ONE 11:e0156369

68. Batchelor TT, Gerstner ER, Ye X et al (2017) Feasibility, phase I, and phase II studies of tandutinib, an oral platelet-derived growth factor receptor-beta tyrosine kinase inhibitor, in patients with recurrent glioblastoma. Neuro Oncol 19:567-575

69. Aiken R, Axelson M, Harmenberg J, Klockare M, Larsson O, Wassberg C (2017) Phase I clinical trial of AXL1717 for treatment of relapsed malignant astrocytomas: analysis of dose and response. Oncotarget 8:81501-81510

70. van den Bent M, Gan HK, Lassman AB et al (2017) Efficacy of depatuxizumab mafodotin (ABT-414) monotherapy in patients with EGFR-amplified, recurrent glioblastoma: results from a multi-center, international study. Cancer Chemother Pharmacol 80:1209-1217

71. Gilbert MR, Kuhn J, Lamborn KR et al (2012) Cilengitide in patients with recurrent glioblastoma: the results of NABTC 03-02, a phase II trial with measures of treatment delivery. J Neurooncol 106:147-153

72. Schiff D, Jaeckle KA, Anderson SK et al (2018) Phase $1 / 2$ trial of temsirolimus and sorafenib in the treatment of patients with 
recurrent glioblastoma: North Central Cancer Treatment Group Study/Alliance N0572. Cancer 124:1455-1463

73. Jaeckle KA, Hess KR, Yung WK et al (2003) Phase II evaluation of temozolomide and 13-cis-retinoic acid for the treatment of recurrent and progressive malignant glioma: a North American Brain Tumor Consortium study. J Clin Oncol 21:2305-2311

74. Wang L, Liang L, Yang T et al (2017) A pilot clinical study of apatinib plus irinotecan in patients with recurrent high-grade glioma: clinical trial/experimental study. Medicine (Baltimore) 96:e9053

75. Odia Y, Kreisl TN, Aregawi D, Innis EK, Fine HA (2015) A phase II trial of tamoxifen and bortezomib in patients with recurrent malignant gliomas. J Neurooncol 125:191-195

76. Grisanti S, Ferrari VD, Buglione M et al (2019) Second line treatment of recurrent glioblastoma with sunitinib: results of a phase II study and systematic review of literature. J Neurosurg Sci 63:458-467

77. Duerinck J, Du Four S, Bouttens F et al (2018) Randomized phase II trial comparing axitinib with the combination of axitinib and lomustine in patients with recurrent glioblastoma. $\mathrm{J}$ Neurooncol 136:115-125

78. Brandes AA, Carpentier AF, Kesari S et al (2016) A phase II randomized study of galunisertib monotherapy or galunisertib plus lomustine compared with lomustine monotherapy in patients with recurrent glioblastoma. Neuro Oncol 18:1146-1156

79. Blumenthal DT, Rankin C, Stelzer KJ et al (2015) A phase III study of radiation therapy (RT) and $\mathrm{O}(6)$-benzylguanine $+\mathrm{BCNU}$ versus RT and BCNU alone and methylation status in newly diagnosed glioblastoma and gliosarcoma: Southwest Oncology Group (SWOG) study S0001. Int J Clin Oncol 20:650-658

80. Batchelor TT, Mulholland P, Neyns B et al (2013) Phase III randomized trial comparing the efficacy of cediranib as monotherapy, and in combination with lomustine, versus lomustine alone in patients with recurrent glioblastoma. J Clin Oncol 31:3212-3218

81. Dic A, Carrabba G, Lanfranchi G, Menghetti C, Rampini P, Caroli M (2013) Continuous tamoxifen and dose-dense temozolomide in recurrent glioblastoma. Anticancer Res 33:3383-3389

82. Couldwell WT, Hinton DR, Surnock AA et al (1996) Treatment of recurrent malignant gliomas with chronic oral high-dose tamoxifen. Clin Cancer Res 2:619-622

83. Graham CD, Kaza N, Klocke BJ et al (2016) Tamoxifen induces cytotoxic autophagy in glioblastoma. J Neuropathol Exp Neurol 75:946-954

84. Robins HI, Won M, Seiferheld WF et al (2006) Phase 2 trial of radiation plus high-dose tamoxifen for glioblastoma multiforme: RTOG protocol BR-0021. Neuro Oncol 8:47-52

85. Ahmed N, Brawley V, Hegde M et al (2017) HER2-specific chimeric antigen receptor-modified virus-specific $\mathrm{T}$ cells for progressive glioblastoma: a phase 1 dose-escalation trial. JAMA Oncol 3:1094-1101

86. Bloch O, Parsa AT (2014) Heat shock protein peptide complex-96 (HSPPC-96) vaccination for recurrent glioblastoma: a phase II, single arm trial. Neuro Oncol 16:758-759

87. Carpentier A, Laigle-Donadey F, Zohar S et al (2006) Phase 1 trial of a $\mathrm{CpG}$ oligodeoxynucleotide for patients with recurrent glioblastoma. Neuro Oncol 8:60-66

88. Chamberlain MC, Kim BT (2017) Nivolumab for patients with recurrent glioblastoma progressing on bevacizumab: a retrospective case series. J Neurooncol 133:561-569

89. Chang CN, Huang YC, Yang DM et al (2011) A phase I/II clinical trial investigating the adverse and therapeutic effects of a postoperative autologous dendritic cell tumor vaccine in patients with malignant glioma. J Clin Neurosci 18:1048-1054
90. Clavreul A, Piard N, Tanguy JY et al (2010) Autologous tumor cell vaccination plus infusion of GM-CSF by a programmable pump in the treatment of recurrent malignant gliomas. J Clin Neurosci 17:842-848

91. Cloughesy TF, Mochizuki AY, Orpilla JR et al (2019) Neoadjuvant anti-PD-1 immunotherapy promotes a survival benefit with intratumoral and systemic immune responses in recurrent glioblastoma. Nat Med 25:477-486

92. Desjardins A, Gromeier M, Herndon JE 2nd et al (2018) Recurrent glioblastoma treated with recombinant poliovirus. N Engl J Med 379:150-161

93. Dillman RO, Duma CM, Schiltz PM et al (2004) Intracavitary placement of autologous lymphokine-activated killer (LAK) cells after resection of recurrent glioblastoma. J Immunother 27:398-404

94. Fakhrai H, Mantil JC, Liu L et al (2006) Phase I clinical trial of a TGF-beta antisense-modified tumor cell vaccine in patients with advanced glioma. Cancer Gene Ther 13:1052-1060

95. Fenstermaker RA, Ciesielski MJ, Qiu J et al (2016) Clinical study of a survivin long peptide vaccine (SurVaxM) in patients with recurrent malignant glioma. Cancer Immunol Immunother 65:1339-1352

96. Hau P, Jachimczak P, Schlingensiepen R et al (2007) Inhibition of TGF-beta2 with AP 12009 in recurrent malignant gliomas: from preclinical to phase I/II studies. Oligonucleotides 17:201-212

97. Lukas RV, Rodon J, Becker K et al (2018) Clinical activity and safety of atezolizumab in patients with recurrent glioblastoma. J Neurooncol 140:317-328

98. Mantica M, Pritchard A, Lieberman F, Drappatz J (2018) Retrospective study of nivolumab for patients with recurrent high grade gliomas. J Neurooncol 139:625-631

99. Narita Y, Arakawa Y, Yamasaki F et al (2019) A randomized, double-blind, phase III trial of personalized peptide vaccination for recurrent glioblastoma. Neuro Oncol 21:348-359

100. Plautz GE, Barnett GH, Miller DW et al (1998) Systemic T cell adoptive immunotherapy of malignant gliomas. J Neurosurg 89:42-51

101. Reiss SN, Yerram P, Modelevsky L, Grommes C (2017) Retrospective review of safety and efficacy of programmed cell death-1 inhibitors in refractory high grade gliomas. J Immunother Cancer 5:99

102. Sakai K, Shimodaira S, Maejima S et al (2015) Dendritic cellbased immunotherapy targeting Wilms' tumor 1 in patients with recurrent malignant glioma. J Neurosurg 123:989-997

103. Schalper KA, Rodriguez-Ruiz ME, Diez-Valle R et al (2019) Neoadjuvant nivolumab modifies the tumor immune microenvironment in resectable glioblastoma. Nat Med 25:470-476

104. Schijns VE, Pretto C, Devillers L et al (2015) First clinical results of a personalized immunotherapeutic vaccine against recurrent, incompletely resected, treatment-resistant glioblastoma multiforme (GBM) tumors, based on combined allo- and auto-immune tumor reactivity. Vaccine 33:2690-2696

105. Schuessler A, Smith C, Beagley L et al (2014) Autologous T-cell therapy for cytomegalovirus as a consolidative treatment for recurrent glioblastoma. Cancer Res 74:3466-3476

106. Shibao S, Ueda R, Saito K et al (2018) A pilot study of peptide vaccines for VEGF receptor 1 and 2 in patients with recurrent/ progressive high grade glioma. Oncotarget 9:21569-21579

107. Tanaka M, Tsuno NH, Fujii T, Todo T, Saito N, Takahashi K (2013) Human umbilical vein endothelial cell vaccine therapy in patients with recurrent glioblastoma. Cancer Sci 104:200-205

108. Terasaki M, Shibui S, Narita Y et al (2011) Phase I trial of a personalized peptide vaccine for patients positive for human leukocyte antigen-A24 with recurrent or progressive glioblastoma multiforme. J Clin Oncol 29:337-344 
109. Tsuboi A, Hashimoto N, Fujiki F et al (2019) A phase I clinical study of a cocktail vaccine of Wilms' tumor 1 (WT1) HLA class I and II peptides for recurrent malignant glioma. Cancer Immunol Immunother 68:331-340

110. Tsuboi K, Saijo K, Ishikawa E et al (2003) Effects of local injection of ex vivo expanded autologous tumor-specific T lymphocytes in cases with recurrent malignant gliomas. Clin Cancer Res 9:3294-3302

111. Yamanaka R, Homma J, Yajima N et al (2005) Clinical evaluation of dendritic cell vaccination for patients with recurrent glioma: results of a clinical phase I/II trial. Clin Cancer Res 11:4160-4167

112. Hunn MK, Bauer E, Wood CE et al (2015) Dendritic cell vaccination combined with temozolomide retreatment: results of a phase I trial in patients with recurrent glioblastoma multiforme. J Neurooncol 121:319-329

113. Akasaki Y, Kikuchi T, Homma S et al (2016) Phase I/II trial of combination of temozolomide chemotherapy and immunotherapy with fusions of dendritic and glioma cells in patients with glioblastoma. Cancer Immunol Immunother 65:1499-1509

114. Bota DA, Chung J, Dandekar M et al (2018) Phase II study of ERC1671 plus bevacizumab versus bevacizumab plus placebo in recurrent glioblastoma: interim results and correlations with CD4(+) T-lymphocyte counts. CNS Oncol 7:CNS22

Publisher's Note Springer Nature remains neutral with regard to jurisdictional claims in published maps and institutional affiliations.

\section{Authors and Affiliations}

\section{Evan Winograd ${ }^{1} \cdot$ Isabelle Germano $^{2} \cdot$ Patrick Wen $^{3} \cdot$ Jeffrey J. Olson ${ }^{4} \cdot$ D. Ryan Ormond ${ }^{1,5}$ (i)}

D. Ryan Ormond

David.ormond@cuanschutz.edu

1 Department of Neurosurgery, University of Colorado School of Medicine, Aurora, CO, USA

2 Department of Neurosurgery, The Mount Sinai Hospital, New York, NY, USA

3 Center for Neuro-Oncology, Dana-Farber Cancer Institute, Harvard Medical School, Boston, MA, USA
4 Department of Neurosurgery, Emory University School of Medicine, Atlanta, GA, USA

5 Department of Neurosurgery, University of Colorado Anschutz Medical Campus, 12631 E. 17th Ave., Mail Stop C307, Aurora, CO 80045, USA 